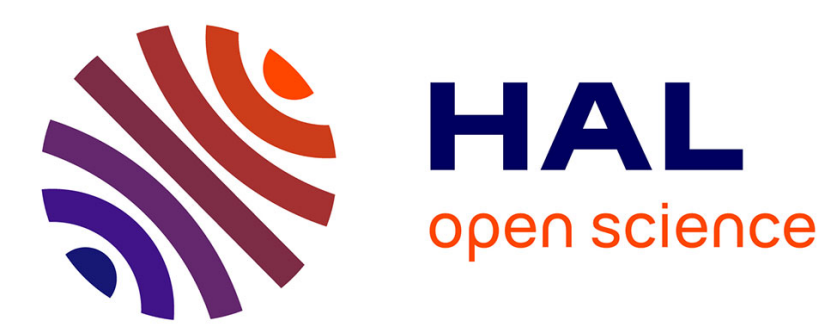

\title{
Évaluation musculaire clinique et IRM après abord mini invasif antérolatéral pour arthroplastie totale de hanche
}

\author{
Simon Mouchel
}

\section{To cite this version:}

Simon Mouchel. Évaluation musculaire clinique et IRM après abord mini invasif antérolatéral pour arthroplastie totale de hanche. Médecine humaine et pathologie. 2013. dumas-00926102

\section{HAL Id: dumas-00926102 https://dumas.ccsd.cnrs.fr/dumas-00926102}

Submitted on 9 Jan 2014

HAL is a multi-disciplinary open access archive for the deposit and dissemination of scientific research documents, whether they are published or not. The documents may come from teaching and research institutions in France or abroad, or from public or private research centers.
L'archive ouverte pluridisciplinaire $\mathbf{H A L}$, est destinée au dépôt et à la diffusion de documents scientifiques de niveau recherche, publiés ou non, émanant des établissements d'enseignement et de recherche français ou étrangers, des laboratoires publics ou privés. 


\title{
THESE POUR LE DOCTORAT EN MEDECINE
}

(Diplôme d'Etat)

PAR

MOUCHEL Simon

Né le 28/02/1983 à Harfleur

PRESENTEE ET SOUTENUE PUBLIQUEMENT LE 22 OCTOBRE 2013

\section{EVALUATION MUSCULAIRE CLINIQUE ET IRM APRES ABORD MINI INVASIF ANTERO LATERAL POUR ARTHROPLASTIE TOTALE DE HANCHE}

\author{
PRESIDENT DU JURY \\ Monsieur le Professeur Franck DUJARDIN \\ DIRECTEUR DE THESE \\ Monsieur le Docteur Jean MATSOUKIS \\ MEMBRES DU JURY \\ Monsieur le Professeur Fabrice DUPARC \\ Monsieur le Professeur Christophe PEILLON
}




\section{Au Professeur Franck Dujardin}

Vous me faites l'honneur de présider ce jury. Vous nous enseignez une chirurgie remarquable et de qualité. Vous savez garder la maitrise du geste technique en toute circonstance même lorsque la situation est parfois très délicate. Je suis fier d'avoir pu écouter, observer et participer avec attention à cette école de chirurgie. Veuillez trouver ici l'expression de ma gratitude et de mon respect.

\section{Au Docteur Jean Matsoukis}

Vous me faites l'honneur de diriger ce travail. Votre rigueur et habileté technique dans chaque geste et indication forcent le respect. Pour votre enseignement, votre soutien et votre disponibilité permanente, professionnelle comme personnelle : merci. C'est avec une grande fierté et reconnaissance que je vous adresse dans cette thèse, le témoignage de mon profond respect, et de mes sincères remerciements.

\section{Au Professeur Fabrice Duparc}

Vous me faites l'honneur de juger ce travail. Votre enseignement de qualité en première année de médecine m'a donné le goût et l'envie d'avancer sur ce chemin. Votre rigueur chirurgicale durant cet internat m'a beaucoup apporté et stimulé. Veuillez trouver dans ce travail le témoignage de mes sincères remerciements.

\section{Au Professeur Christophe Peillon}

Vous me faites l'honneur de juger ce travail. Je vous remercie d'avoir accepté de participer à ce jury de thèse. Pour votre disponibilité et votre expertise chirurgicale, veuillez trouver dans ce travail le témoignage de ma reconnaissance. 
Au Docteur Xavier Roussignol : Je tiens à vous adresser mes sincères remerciements pour la transmission de votre savoir-faire et cette notion de compagnonnage qui m'est chère.

Au Docteur Eric Lenoble : Je vous remercie pour votre gentillesse et participation à l'enseignement lors des staffs et autres réunions de service.

Au Docteur Jacques-Marie Adam : Tu m'as accueilli, soutenu et enseigné de super moments de vie ! Je garde grâce à toi un super souvenir de cet internat.

Au Docteur Olivier Courage : Je vous adresse mon profond respect. Vous m'avez accueilli au sein de votre équipe et j'ai réellement apprécié cette année de compagnonnage chirurgical. Les journées étaient chargées mais le temps passait trop vite. A mes yeux, vous avez la carrure d'un grand enseignant.

Au Professeur Christophe Hulet : Je vous remercie de m'avoir accueilli durant 6 mois dans votre service. Veuillez recevoir ici toute ma gratitude.

\section{Aux Docteurs J-M Bondeville, Paul Michelin et Valentin}

Lefebvre, sans qui ce travail n'aurait pu se réaliser. Merci pour vos bons conseils, votre disponibilité et votre œil averti. Valentin, j'ai été très heureux d'opérer dans ce bloc avec toi.

Au Docteur Saad Abu Amara : Je vous remercie pour ces nombreux bons moments dans le service et au bloc opératoire passé à vos côtés, pour votre rigueur et pour m'avoir enseigné une partie de votre maitrise chirurgicale. Vous êtes un modèle!

Au Professeur Joël Lechevallier : Je vous remercie pour ce semestre en chirurgie pédiatrique, votre expérience chirurgicale et votre dévouement pour ces enfants, forcent le respect. 
Au Docteur Louay El Ayoubi : Merci pour ta gentillesse et la transmission de ton expérience chirurgicale. Tu as parcouru de nombreux chemins et je tiens à t'exprimer mon plus grand respect.

Au docteur Gilles Balmary : Vous avez marqué le début de mon internat à Vernon, par votre vision éclairée et vos conseils avisés. Je suis très heureux d'avoir pu recevoir votre enseignement de qualité.

A Madame Lydie Lemoal : Je te remercie vivement pour ton investissement dans ce travail et pour m'avoir aidé à retrouver tous les patients.

\section{A toute l'équipe du bloc ortho et du bloc d'urgence avec qui j'ai apprécié ces journées et ces nuits d'internat au bloc opératoire,}

\section{Aux patients qui ont accepté de participer à ce travail,}

\section{A ma famille :}

Mes parents : Merci de m'avoir soutenu et appris à travailler, maman tu m'as donné le goût de l'effort en te levant si tôt chaque matin, papa tu as parcouru le monde. Je vous aime et suis fier de vous. Merci de m'avoir soutenu durant toutes ces années.

Mes grands parents : Papy Bonhomme, tu m'as enseigné tant de choses... Tu m'as toujours soutenu avec mamie, c'est avec un grand honneur que je vous dédie ce travail et c'est avec fierté que je continuerai à enfiler mes sabots et mon «Bleu de travail» chaque matin. Papy Fernand et mamie Paulette, vous avez toujours été présents dans cette grande famille. Pour votre amour, générosité et esprit de famille : je vous aime.

A ma belle famille : Jean, Catherine, Alice (Rral !), Flore (Babé !), Aude (Bernard !), Fredou : Je suis tellement heureux de vous avoir rencontré, je vous aime, et vous fait plein de Gilbert! (Bécots quoi...).

Aux Ardéchois du Mas Tardieu, A Newton et Helios. 


\section{A mes amis :}

Les Rouennais : Les Grigneur : Milouz el toubib de st Jacques (beau geste au dynamomètre !), Hélène l'épicière^^ et le p'tit Raphaël Momo (RRrrrree !), on va à la bobo ce soir ? ça pass... :) ; Les Cerdaki : Clèm Dr en photographie ? Et Alice la gazière^^ ; Aleki et Marco mes potos, Emma et Flo (pour les nombreux footings encore à venir); Les Draillmar : Delphine et Aurélien (merci pour les nombreux prêts de voiture afin de pouvoir aller voir mes patients au Havre) ;

Les Toulousains : les bichons : Jess, la reine du dance floor, Julien ; Cha et Jeff, le maitre de thèse ! (la nouvelle Cal, ça m'aurait bien botté aussi !)

Les Havrais : Yvan, Tigrou, Michou, Verg et Marius, Flo el boulet, Annelaure, Alexboy, Julien, Couscous et Hélène.

\section{A tous mes compagnons de l'internat :}

Chefs et anciens-chefs: Louay: trop fort !, Roberto, Benji (Docteur «Lafève ») l'homme au «truc et astuces » de la chirurgie!, Jacquot pour ses superbes gardes au bloc et ailleurs, Jean-Phil, Fred, Anne, Omar, Julien (Docteur «Belpomme») ton énergie et ton goût pour la transmission écrite du savoir me font envie.

Co-internes devenus chefs, Christian, Gilbert, Vinz «le Baron la»!, Mourad, Manu, Jona, Mathieu, Tonio dit «Lover», Beber, Ouaïpi et Thomas le fin kiteux.

Collègues de l'Orthopower : Elodie, Louigi le planchiste, Azad mon poto, Nico («tarissi la bonne soupe! »), Virginie «ma ptite salade niçoise », Yuyu («de l'eau sur les pattes à papa, la loupiote : destination 5F!»), Laure et Laure $^{\wedge \wedge}$, Lapapouille, ce triathlète \& Damien mes compagnons de la Chir Ped, Saïd, Antoine, Sigou qui a déjà sa carte verte du DESC d'ortho, Camille, Charles, Remi, Phana (si tu retrouves ta valise), Amaury, Cédric, Houssem, Jordan, Adrien et Isabelle. Gardez l'esprit d'équipe !

Les plasticiens : Ghani, Ludo, Albane, Débo, Alex, flore

\section{Aux autres teams :}

\section{Anesthésiologistes, Indiens et lanceurs de fléchettes curarisées :} Sinouche (un gros BK, et ça repart!) et Lauranne, sur qui je pourrais toujours compter. 
Pédiatrie : Seb : la pédiatrie ou l'orthopédie : «entre les deux mon cœur balance », Damien : l'homme au vélo pliable et pro de la négos., les urologues David, Benoit, et Iadd le futur plasticien.

Vasculaires \& thoracique : les 2 Marie (lebrax/layné, dazzou), Sabrinou, Alex : n'oubliez jamais ce que vous a transmis JMB dit le king. Marion B. qui m'a relu et corrigé, entre 2 aortes : merci pour cette dernière garde avec le patron et le pontage aorto mésentérique de minuit : c'était pas dla chirurgie de surface !

Les Caennais : Etienne qui m'a accueilli, Thibault le footeux, Michmich, Mathieu, Valentin, Victo, Tony, tiP...

A L.H. : Avec sa plage de GROS grains de sables (encore un peu jeune !), son soleil (entre deux averses rafraichissantes), ses soirées, les amis, la famille et bientôt un super Job !

A mes héros : Yoda et Vador qui ont trouvé la «Force et l'Honneur », Mulder et Scully qui ont résumé de nombreux cours de philo: «la vérité est ailleurs!», G. Brassens «l'énergumène » et insoumis, Gandalf et Gollum pour leur magie...

A ma femme, Diane : Pour ta patience et ton amour, je suis fier et heureux de pouvoir traverser la vie à tes côtés. Je t'aime, j'espère que notre famille va bientôt s'agrandir, j'ai hâte... 
L'auteur déclare n'avoir aucun conflit d'intérêt pour aucun

fabriquant ou industriel décrit dans ce travail 
Par délibération en date du 3 mars 1967, l’Université de Rouen et l'UFR de Médecine et de Pharmacie de Rouen n'entendent donner aucune approbation ni improbation aux opinions émises dans cette thèse. Ces opinions sont propres à leurs auteurs. 
ANNEE UNIVERSITAIRE 2012 - 2013

U.F.R. DE MEDECINE-PHARMACIE DE ROUEN

DOYEN :

ASSESSEURS :

DOYENS HONORAIRES :

PROFESSEURS HONORAIRES :
Professeur Pierre FREGER

Professeur Michel GUERBET

Professeur Benoit VEBER

Professeur Pascal JOLY

Professeur Bernard PROUST

Professeurs J. BORDE - Ph. LAURET - H. PIGUET - C. THUILLEZ

MM. M-P AUGUSTIN - J.ANDRIEU-GUITRANCOURT M.BENOZIO - J.BORDE - Ph. BRASSEUR - R. COLIN - E. COMOY - J. DALION -. DESHAYES - C. FESSARD - J.P FILLASTRE - P.FRIGOT -J. GARNIER - J. HEMET - B. HILLEMAND - G. HUMBERT - J.M. JOUANY - $\mathbf{R}$. LAUMONIER - Ph. LAURET - M. LE FUR - J.P. LEMERCIER - J.P LEMOINE - MIS MAGARD - MM. B. MAITROT - M. MAISONNET - F. MATRAY P.MITROFANOFF - Mme A. M. ORECCHIONI - P. PASQUIS H.PIGUET - M.SAMSON - Mme SAMSON-DOLLFUS - J.C. SCHRUB - R.SOYER - B.TARDIF -.TESTART - J.M. THOMINE - C. THUILLEZ - P.TRON - C.WINCKLER L.M.WOLF

I - MEDECINE

\section{PROFESSEURS}

M. Frédéric ANSELME

Mme Isabelle AUQUIT AUCKBUR

M. Bruno BACHY

M. Fabrice BAUER

Mme Soumeya BEKRI

M. Jacques BENICHOU

M. Jean-Paul BESSOU

Mme Françoise BEURET-BLANQUART

M. Guy BONMARCHAND

M. Olivier BOYER

M. Jean-François CAILLARD (Surnombre) HCN

M. François CARON

M. Philippe CHASSAGNE

M. Vincent COMPERE
$\mathrm{HCN} \quad$ Cardiologie

$\mathrm{HCN} \quad$ Chirurgie Plastique

$\mathrm{HCN} \quad$ Chirurgie pédiatrique

$\mathrm{HCN} \quad$ Cardiologie

$\mathrm{HCN} \quad$ Biochimie et Biologie Moléculaire

$\mathrm{HCN} \quad$ Biostatistiques et informatique médicale

$\mathrm{HCN} \quad$ Chirurgie thoracique et cardio-vasculaire

CRMPR Médecine physique et de réadaptation

$\mathrm{HCN} \quad$ Réanimation médicale

UFR Immunologie

$\mathrm{HCN} \quad$ Maladies infectieuses et tropicales

HB Médecine interne (Gériatrie)

$\mathrm{HCN} \quad$ Anesthésiologie et réanimation chirurgicale 
M. Alain CRIBIER (Surnombre)

M. Antoine CUVELIER

M. Pierre CZERNICHOW

M. Jean - Nicolas DACHER

M. Stéfan DARMONI

M. Pierre DECHELOTTE

Mme Danièle DEHESDIN

M. Jean DOUCET

M. Bernard DUBRAY

M. Philippe DUCROTTE

M. Frank DUJARDIN

M. Fabrice DUPARC

M. Bertrand DUREUIL

Mle Hélène ELTCHANINOFF

M. Thierry FREBOURG

M. Pierre FREGER

M. Jean François GEHANNO

M. Emmanuel GERARDIN

Mme Priscille GERARDIN

M. Michel GODIN

M. Philippe GRISE

M. Didier HANNEQUIN

M. Fabrice JARDIN

M. Luc-Marie JOLY

M. Pascal JOLY

M. Jean-Marc KUHN

Mme Annie LAQUERRIERE

M. Vincent LAUDENBACH

M. Joël LECHEVALLIER

M. Hervé LEFEBVRE

M. Thierry LEQUERRE

M. Eric LEREBOURS

Mle Anne-Marie LEROI

M. Hervé LEVESQUE

Mme Agnès LIARD-ZMUDA

M. Pierre Yves LITZLER

M. Bertrand MACE

M. Eric MALLET (Surnombre)

M. Christophe MARGUET

Mle Isabelle MARIE

M. Jean-Paul MARIE

M. LoÏc MARPEAU

M. Stéphane MARRET
$\mathrm{HCN} \quad$ Cardiologie

HB Pneumologie

$\mathrm{HCH} \quad$ Epidémiologie, économie de la santé

$\mathrm{HCN} \quad$ Radiologie et Imagerie Médicale

HCN Informatique Médicale/Techniques de com.

HCN Nutrition

$\mathrm{HCN}$ Oto-Rhino-Laryngologie

HB Thérapeutique/Médecine - Interne - Gériatrie.

CB Radiothérapie

$\mathrm{HCN} \quad$ Hépato - Gastro - Entérologie

$\mathrm{HCN} \quad$ Chirurgie Orthopédique - Traumatologique

HCN Anatomie - Chirurgie Orthopédique et Traum.

$\mathrm{HCN}$ Anesthésiologie et réanimation chirurgicale

$\mathrm{HCN}$ Cardiologie

UFR Génétique

$\mathrm{HCN}$ Anatomie/Neurochirurgie

$\mathrm{HCN} \quad$ Médecine et Santé au Travail

$\mathrm{HCN}$ Imagerie Médicale

$\mathrm{HCN} \quad$ Pédopsychiatrie

HB Néphrologie

$\mathrm{HCN} \quad$ Urologie

$\mathrm{HCN} \quad$ Neurologie

$\mathrm{CB} \quad$ Hématologie

$\mathrm{HCN} \quad$ Médecine d'urgence

$\mathrm{HCN}$ Dermato - vénéréologie

HB Endocrinologie et maladies métaboliques

$\mathrm{HCN} \quad$ Anatomie cytologie pathologiques

$\mathrm{HCN}$ Anesthésie et réanimation chirurgicale

$\mathrm{HCN} \quad$ Chirurgie infantile

HB Endocrinologie et maladies métaboliques

$\mathrm{HB} \quad$ Rhumatologie

HCN Nutrition

$\mathrm{HCN}$ Physiologie

HB Médecine interne

$\mathrm{HCN} \quad$ Chirurgie Infantile

$\mathrm{HCN} \quad$ Chirurgie Cardiaque

HCN Histologie, embryologie, cytogénétique

$\mathrm{HCN}$ Pédiatrie

$\mathrm{HCN}$ Pédiatrie

HB Médecine Interne

HCN ORL

HCN Gynécologie - obstétrique

$\mathrm{HCN}$ Pédiatrie 


\begin{tabular}{|c|c|c|}
\hline Mme Véronique MERLE & $\mathrm{HCN}$ & Epidémiologie \\
\hline M. Pierre MICHEL & $\mathrm{HCN}$ & Hépato - Gastro - Entérologie \\
\hline M. Francis MICHOT & $\mathrm{HCN}$ & Chirurgie digestive \\
\hline M. Bruno MIHOUT (Surnombre) & $\mathrm{HCN}$ & Neurologie \\
\hline M. Jean-François MUIR & $\mathrm{HB}$ & Pneumologie \\
\hline M. Marc MURAINE & $\mathrm{HCN}$ & Ophtalmologie \\
\hline M. Philippe MUSETTE & $\mathrm{HCN}$ & Dermatologie - Vénéréologie \\
\hline M. Christophe PEILLON & $\mathrm{HCN}$ & Chirurgie générale \\
\hline M. Jean-Marc PERON & $\mathrm{HCN}$ & Stomatologie et chirurgie maxillo-faciale \\
\hline M. Christian PFISTER & $\mathrm{HCN}$ & Urologie \\
\hline M. Jean-Christophe PLANTIER & $\mathrm{HCN}$ & Bactériologie - Virologie \\
\hline M. Didier PLISSONNIER & $\mathrm{HCN}$ & Chirurgie vasculaire \\
\hline M. Bernard PROUST & $\mathrm{HCN}$ & Médecine légale \\
\hline M. François PROUST & $\mathrm{HCN}$ & Neurochirurgie \\
\hline Mme Nathalie RIVES & $\mathrm{HCN}$ & Biologie et méd. du dévelop. et de la reprod. \\
\hline M. J.-Christophe RICHARD (Mise en dispo) & $\mathrm{HCN}$ & Réanimation Médicale, Médecine d'urgence \\
\hline M. Horace ROMAN & $\mathrm{HCN}$ & Gynécologie Obstétrique \\
\hline M. Jean-Christophe SABOURIN & $\mathrm{HCN}$ & Anatomie - Pathologie \\
\hline M. Guillaume SAVOYE & $\mathrm{HCN}$ & Hépato - Gastro \\
\hline Mme Céline SAVOYE - COLLET & $\mathrm{HCN}$ & Imagerie Médicale \\
\hline M. Michel SCOTTE & $\mathrm{HCN}$ & Chirurgie digestive \\
\hline Mme Fabienne TAMION & $\mathrm{HCN}$ & Thérapeutique \\
\hline Mle Florence THIBAUT & $\mathrm{HCN}$ & Psychiatrie d'adultes \\
\hline M. Luc THIBERVILLE & $\mathrm{HCN}$ & Pneumologie \\
\hline M. Christian THUILLEZ & $\mathrm{HB}$ & Pharmacologie \\
\hline M. Hervé TILLY & $\mathrm{CB}$ & Hématologie et transfusion \\
\hline M. François TRON (Surnombre) & UFR & Immunologie \\
\hline M. Jean-Jacques TUECH & $\mathrm{HCN}$ & Chirurgie digestive \\
\hline M. Jean-Pierre VANNIER & $\mathrm{HCN}$ & Pédiatrie génétique \\
\hline M. Benoît VEBER & $\mathrm{HCN}$ & Anesthésiologie Réanimation chirurgicale \\
\hline M. Pierre VERA & C.B & Biophysique et traitement de l'image \\
\hline M. Eric VERIN & CRMPR & Médecine physique et de réadaptation \\
\hline M. Eric VERSPYCK & $\mathrm{HCN}$ & Gynécologie obstétrique \\
\hline M. Olivier VITTECOQ & $\mathrm{HB}$ & Rhumatologie \\
\hline M. Jacques WEBER & $\mathrm{HCN}$ & Physiologie \\
\hline
\end{tabular}


MAITRES DE CONFERENCES

$\begin{array}{lll}\text { Mme Noëlle BARBIER-FREBOURG } & \text { HCN } & \text { Bactériologie - Virologie } \\ \text { M. Jeremy BELLIEN } & \mathrm{HCN} & \text { Pharmacologie } \\ \text { Mme Carole BRASSE LAGNEL } & \mathrm{HCN} & \text { Biochimie } \\ \text { Mme Mireille CASTANET } & \mathrm{HCN} & \text { Pédiatrie } \\ \text { M. Gérard BUCHONNET } & \mathrm{HCN} & \text { Hématologie } \\ \text { Mme Nathalie CHASTAN } & \mathrm{HCN} & \text { Physiologie } \\ \text { Mme Sophie CLAEYSSENS } & \mathrm{HCN} & \text { Biochimie et biologie moléculaire } \\ \text { M. Moïse COEFFIER } & \mathrm{HCN} & \text { Nutrition } \\ \text { M. Manuel ETIENNE } & \mathrm{HCN} & \text { Maladies infectieuses et tropicales } \\ \text { M. Guillaume GOURCEROL } & \mathrm{HCN} & \text { Physiologie } \\ \text { Mme Catherine HAAS-HUBSCHER } & \mathrm{HCN} & \text { Anesthésie - Réanimation chirurgicale } \\ \text { M. Serge JACQUOT } & \mathrm{UFR} & \text { Immunologie } \\ \text { M. Joël LADNER } & \mathrm{HCN} & \text { Epidémiologie, économie de la santé } \\ \text { M. Jean-Baptiste LATOUCHE } & \mathrm{UFR} & \text { Biologie Cellulaire } \\ \text { Mme Lucie MARECHAL-GUYANT } & \mathrm{HCN} & \text { Neurologie } \\ \text { M. Thomas MOUREZ } & \mathrm{HCN} & \text { Bactériologie } \\ \text { M. Jean-François MENARD } & \mathrm{HCN} & \text { Biophysique } \\ \text { Mme Muriel QUILLARD } & \mathrm{HCN} & \text { Biochimie et Biologie moléculaire } \\ \text { M. Vincent RICHARD } & \text { UFR } & \text { Pharmacologie } \\ \text { M. Francis ROUSSEL } & \mathrm{HCN} & \text { Histologie, embryologie, cytogénétique } \\ \text { Mme Pascale SAUGIER-VEBER } & \mathrm{HCN} & \text { Génétique } \\ \text { Mme Anne-Claire TOBENAS-DUJARDIN } & \mathrm{HCN} & \text { Anatomie }\end{array}$

PROFESSEUR AGREGE OU CERTIFIE

Mme Dominique LANIEZ

Mme Cristina BADULESCU
UFR Anglais

UFR Communication 


\section{PROFESSEURS}

M. Thierry BESSON

M. Jean-Jacques BONNET

M. Roland CAPRON (PU-PH)

$M$. Jean COSTENTIN (Professeur émérite)

Mme Isabelle DUBUS

M. LoÏc FAVENNEC (PU-PH)

$M$. Jean Pierre GOULLE

M. Michel GUERBET

M. Olivier LAFONT

Mme Isabelle LEROUX

M. Paul MULDER

Mme Martine PESTEL-CARON (PU-PH)

Mme Elisabeth SEGUIN

$M$ Jean-Marie VAUGEOIS

M. Philippe VERITE

\section{MAITRES DE CONFERENCES}

Mle Cécile BARBOT

Mme Dominique BOUCHER

$M$. Frédéric BOUNOURE

M. Abdeslam CHAGRAOUI

M. Jean CHASTANG

Mme Marie Catherine CONCE-CHEMTOB

Mme Elizabeth CHOSSON

Mle Cécile CORBIERE

M. Eric DITTMAR

Mme Nathalie DOURMAP

Mle Isabelle DUBUC

Mme Roseline DUCLOS

M. Abdelhakim ELOMRI

M. François ESTOUR

M. Gilles GARGALA (MCU-PH)

Mme Najla GHARBI

Mle Marie-Laure GROULT

M. Hervé HUE
Chimie Thérapeutique

Pharmacologie

Biophysique

Pharmacologie

Biochimie

Parasitologie

Toxicologie

Toxicologie

Chimie organique

Physiologie

Sciences du médicament

Microbiologie

Pharmacognosie

Pharmacologie

Chimie analytique
Chimie Générale et Minérale

Pharmacologie

Pharmacie Galénique

Physiologie

Biomathématiques

Législation pharmac. et écono. de la santé

Botanique

Biochimie

Biophysique

Pharmacologie

Pharmacologie

Pharmacie Galénique

Pharmacognosie

Chimie Organique

Parasitologie

Chimie analytique

Botanique

Biophysique et Mathématiques 
Mme Laetitia LE GOFF

Mme Hong LU

Mme Sabine MENAGER

Mme Christelle MONTEIL

M. Mohamed SKIBA

Mme Malika SKIBA

Mme Christine THARASSE

M. Rémi VARIN (MCU-PH)

M. Frédéric ZIEGLER

PROFESSEUR ASSOCIE

Mme Sandrine PANCHOU

PROFESSEUR CONTRACTUEL

Mme Elizabeth DE PAOLIS
Parasitologie Immunologie

Biologie

Chimie organique

Toxicologie

Pharmacie Galénique

Pharmacie Galénique

Chimie thérapeutique

Pharmacie Hospitalière

Biochimie

Pharmacie Officinale

\section{ATTACHE TEMPORAIRE D'ENSEIGNEMENT ET DE RECHERCHE}

M. Mazim MEKAOUI

Mlle Virginie OXARAN

M. Romy RAZAKANDRAINIBE
Anglais 


\section{PROFESSEURS}

M. Jean-Loup HERMIL

UFR

Médecine générale

PROFESSEURS ASSOCIES A MI-TEMPS :

$\begin{array}{lll}\text { M. Pierre FAINSILBER } & \text { UFR } & \text { Médecine générale } \\ \text { M. Alain MERCIER } & \text { UFR } & \text { Médecine générale } \\ \text { M. Philippe NGUYEN THANH } & \text { UFR } & \text { Médecine générale }\end{array}$

MAITRE DE CONFERENCES ASSOCIE A MI-TEMPS :

M Emmanuel LEFEBVRE

Mme Elisabeth MAUVIARD

Mme Marie Thérèse THUEUX
UFR Médecine générale

UFR Médecine générale

UFR Médecine générale

CHEF DES SERVICES ADMINISTRATIFS : Mme Véronique DELAFONTAIN

HCN - Hôpital Charles Nicolle HB - Hôpital de BOIS GUILLAUME

CB - Centre HENRI BECQUEREL CHS - Centre Hospitalier Spécialisé du Rouvray CRMPR - Centre Régional de Médecine Physique et de Réadaptation 
Melle Cécile BARBOT

M. Thierry BESSON

M. Roland CAPRON

M Jean CHASTANG

Mme Marie-Catherine CONCE-CHEMTOB

Mle Elisabeth CHOSsON

M. Jean-Jacques BONNET

Mme Isabelle DUBUS

M. LoÏc FAVENNEC

M. Michel GUERBET

M. Olivier LAFONT

Mme Isabelle LEROUX-NICOLLET

Mme Martine PESTEL-CARON

Mme Elisabeth SEGUIN

M. Mohamed SKIBA

M. Philippe VERITE
Chimie Générale et Minérale

Chimie thérapeutique

Biophysique

Mathématiques

Législation, Economie de la Santé

Botanique

Pharmacodynamie

Biochimie

Parasitologie

Toxicologie

Chimie organique

Physiologie

Microbiologie

Pharmacognosie

Pharmacie Galénique

Chimie analytique 


\section{ENSEIGNANTS MONO-APPARTENANTS}

MAITRES DE CONFERENCES

M. Sahil ADRIOUCH

Biochimie et biologie moléculaire

(Unité Inserm 905)

Mme Gaëlle BOUGEARD-DENOYELLE

Biochimie et biologie moléculaire (UMR 1079)

Mme Carine CLEREN

Neurosciences (Néovasc)

Mme Pascaline GAILDRAT

Génétique moléculaire humaine (UMR 1079)

M. Antoine OUVRARD-PASCAUD

Physiologie (Unité Inserm 1076)

Mme Isabelle TOURNIER

Biochimie (UMR 1079)

PROFESSEURS DES UNIVERSITES

M. Serguei FETISSOV

Physiologie (Groupe ADEN)

Mme Su RUAN

Génie Informatique 


\section{Table des matières}

1. INTRODUCTION

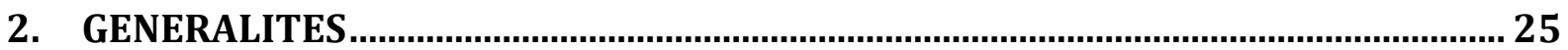

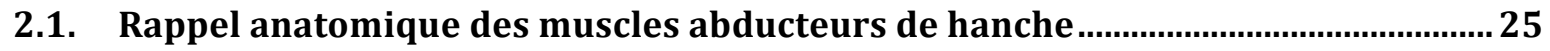

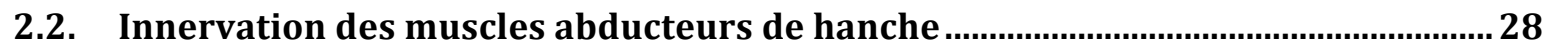

2.3. Risques lésionnels théoriques dans la VALMI ......................................................... 31

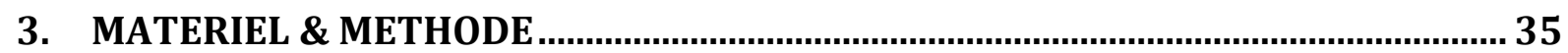

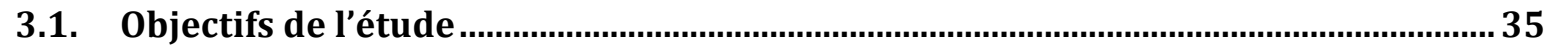

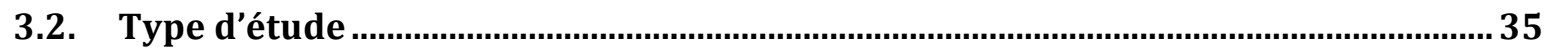

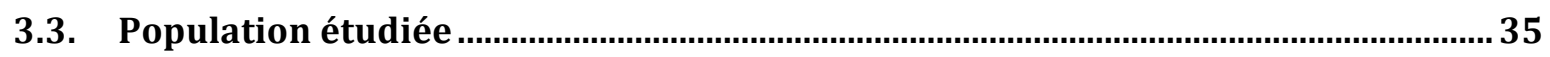

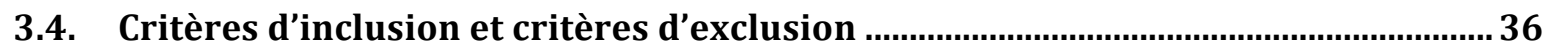

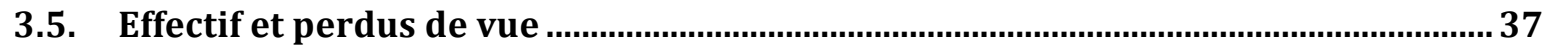

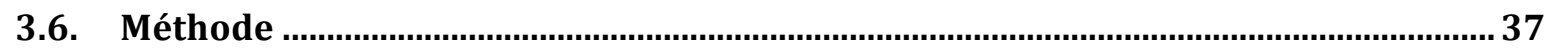

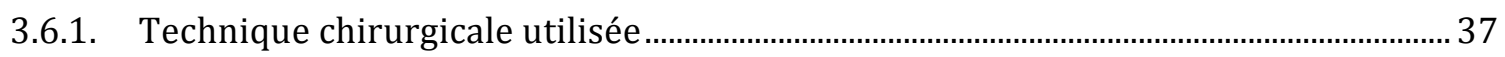

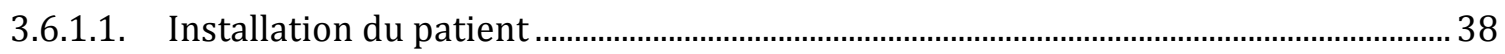

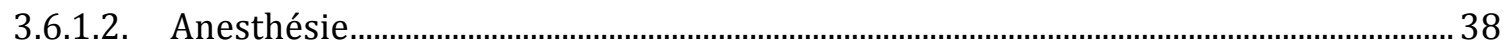

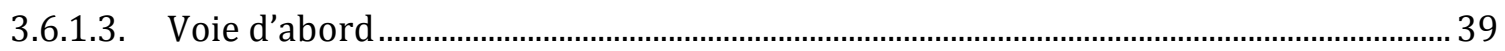

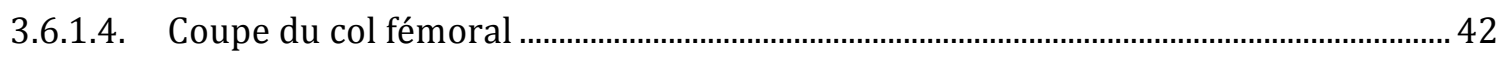

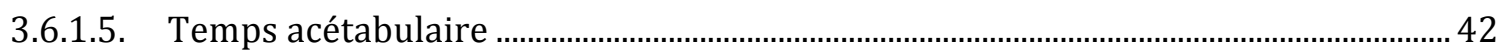

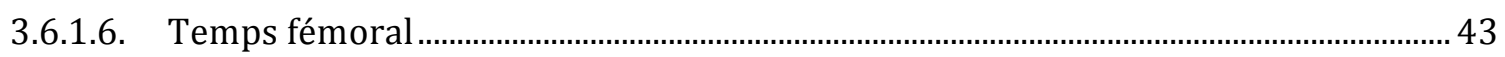

3.6.1.7. Fermeture et protocole post opératoire ........................................................................ 44

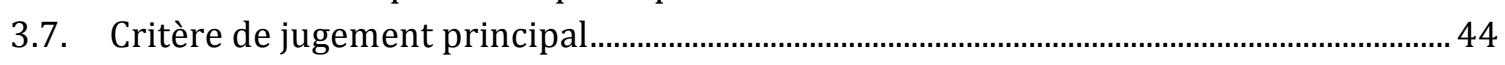

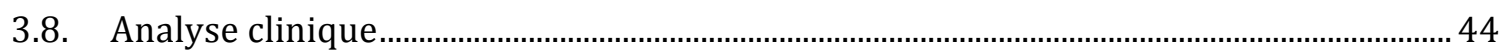

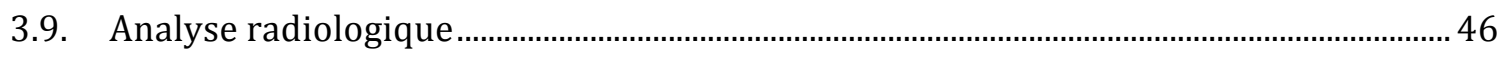

3.9.1. Description du protocole d'imagerie ............................................................................ 46

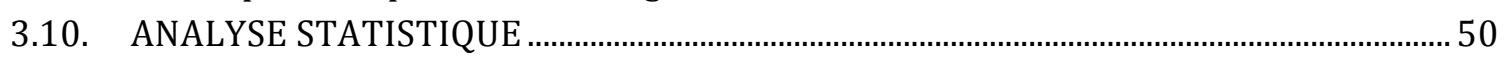

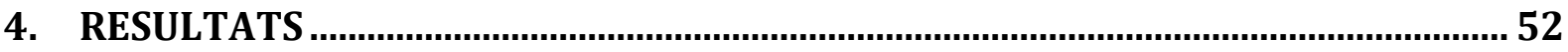

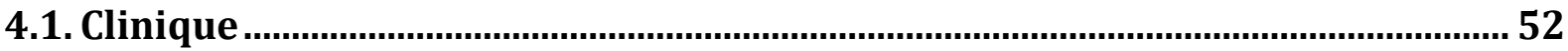

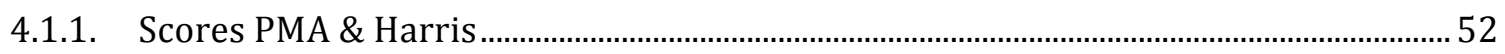

4.1.2. Appui monopodal, Trendelenburg et Force musculaire d'abduction............................. 52

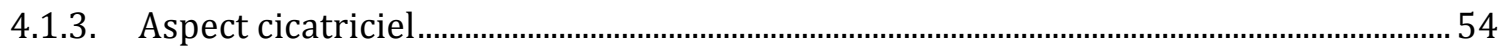

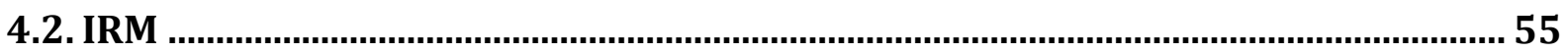

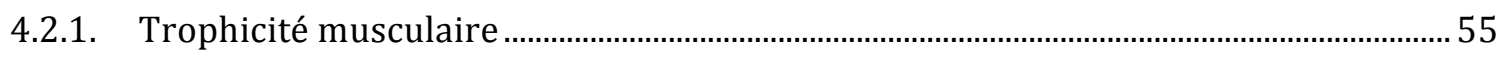

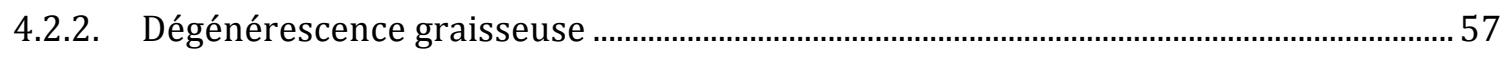

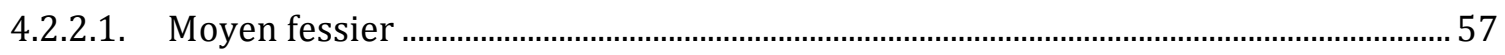

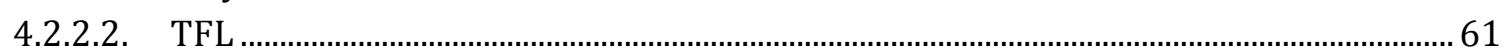

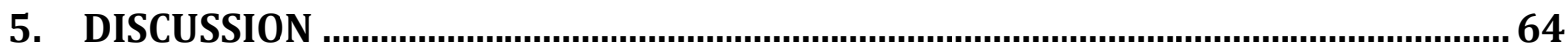

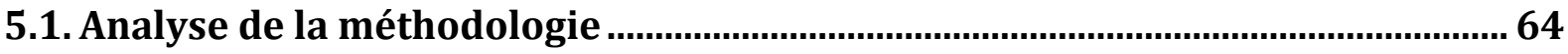

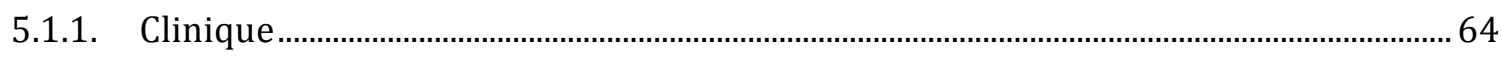

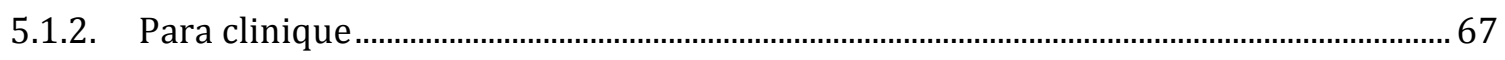

5.1.2.1. Choix de l'imagerie..................................................................................................... 67

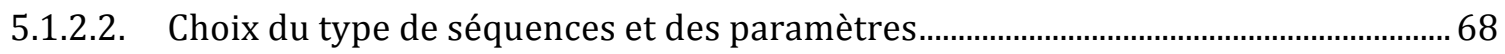

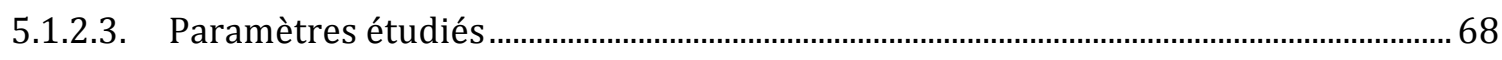

5.1.2.4. Techniques de mesure de la composante contractile et non contractile du muscle 68 
5.1.2.5. Limites de notre étude

5.2. Analyse des lésions musculaires induites par la chirurgie.

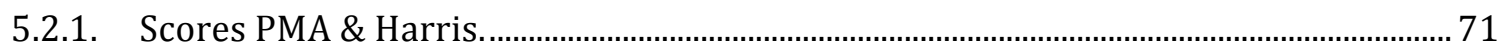

5.2.2. Appui monopodal, boiterie de Trendelenburg et Force musculaire d'abduction..... 74

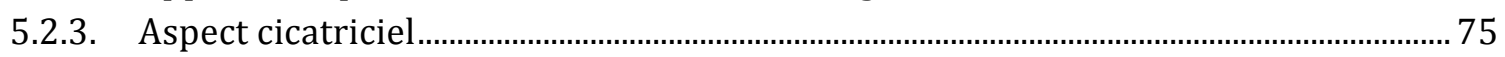

5.2.4. Modification à l'IRM des muscles stabilisateurs du pelvis............................................... 76

5.3. Notions générales de capacité régénérative et de compensation musculaire. 78

5.4. Considérations éthiques et information délivrée aux patients ............................ 80

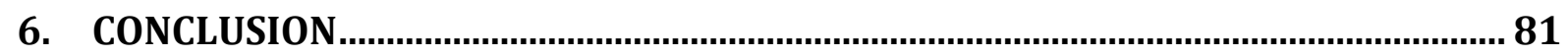

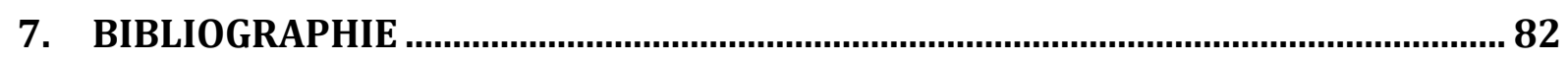

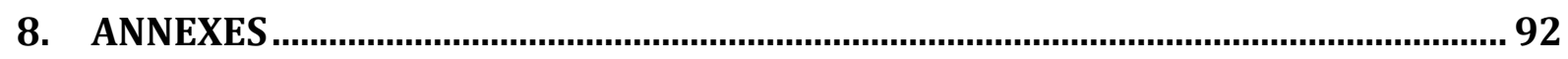

\section{Liste des tableaux :}

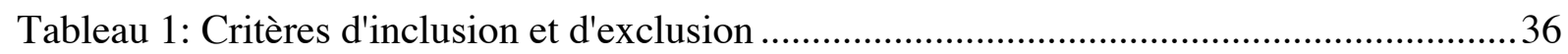

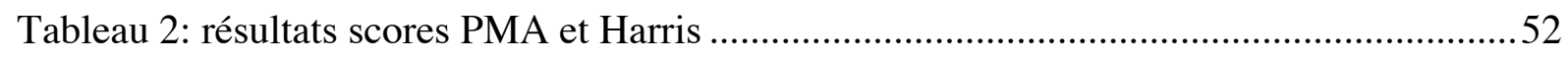

Tableau 3: Résultats appui monopodal (AMP) \& boiterie ......................................................53

Tableau 4: résultats des mesures de la force d'abduction $(\mathrm{N})$.................................................53

Tableau 5: Aires musculaires du moyen fessier à 3 et 12 mois post opératoire........................56

Tableau 6: Aires musculaires du TFL à 3 et 12 mois post opératoire .......................................56

Tableau 7: Dégénérescence graisseuse du $1 / 3$ antérieur du muscle MF à 3 mois post

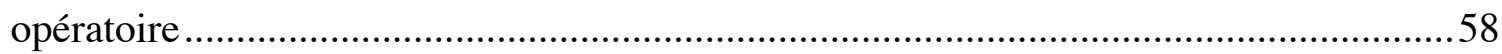

Tableau 8: Dégénérescence graisseuse du1/3 moyen du muscle MF à 3 mois post opératoire58

Tableau 9: Dégénérescence graisseuse du 1/3 postérieur du muscle MF à 3 mois post opératoire

Tableau 10: Dégénérescence graisseuse du 1/3 ant du muscle MF à 12 mois post opératoires

Tableau 11: Dégénérescence graisseuse du 1/3 moyen muscle MF à 12 mois post opératoire59

Tableau 12: Dégénérescence graisseuse du $1 / 3$ postérieur du muscle MF à 12 mois post opératoire

Tableau 13: Dégénérescence graisseuse du muscle tenseur du fascia lata à 3 mois post opératoire

Tableau 14: Dégénérescence graisseuse du muscle tenseur du fascia lata à 12 mois post opératoire

Tableau 15: effectifs des patients présentant un oedème de dénervation du TFL, du côté opéré

Tableau 16: Comparaison des taux de répartition (\%) de la DG du 1/3 antérieure du MF......77

Tableau 17: évaluation chiffrée PMA (Postel, Merle d'Aubigné) ...........................................92

Tableau 18: cotation fonctionnelle de Harris ...................................................................... 93 


\section{Liste des figures :}

Figure 1: Coupes transversales de hanche

Figure 2: Schéma représentant le muscle moyen fessier.......................................................26

Figure 3: Description anatomique du site d'insertion proximal du TFL ................................2 27

Figure 4 : Schémas anatomiques de l'innervation glutéale................................................. 30

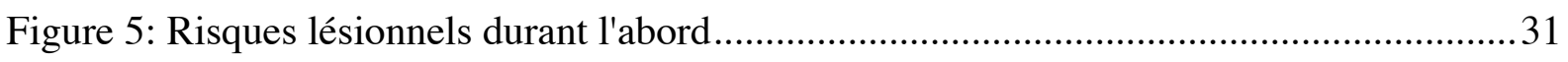

Figure 6: Risques lésionnels durant le temps acétabulaire .................................................. 32

Figure 7: Risques lésionnels durant le temps fémoral ........................................................ 32

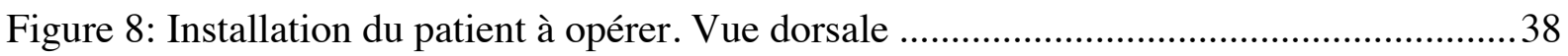

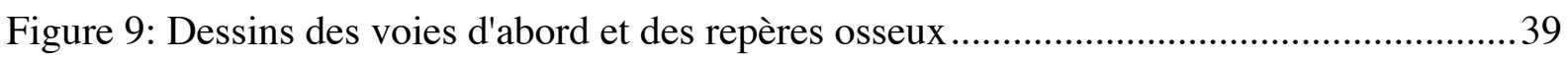

Figure 10: Voie d'abord. Dissection de l'interstice situé entre le muscle MF et le TFL..........41

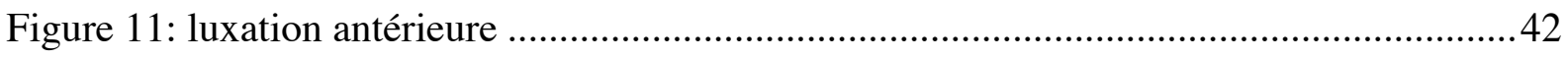

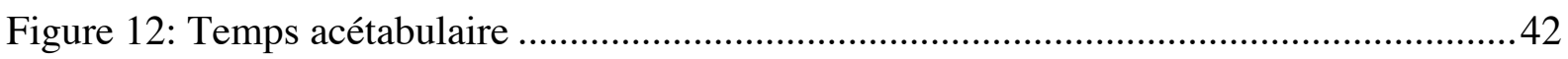

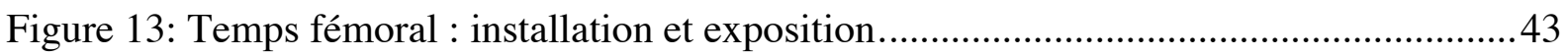

Figure 14: Illustration du dynamomètre utilisé et du testing musculaire................................45

Figure 15: coupe frontale, séquence IRM FAT T1 IDEAL FSE .........................................48

Figure 16: Coupe transversale, séquence IRM FAT T1 IDEAL FSE ...................................49

Figure 17: Coupe transversale, séquence IRM FAT T1 IDEAL FSE ................................... 49

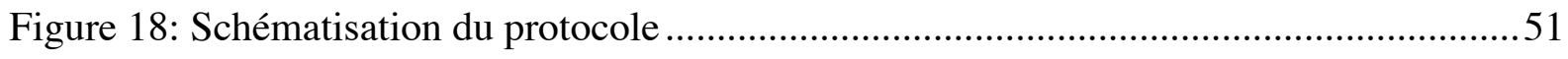

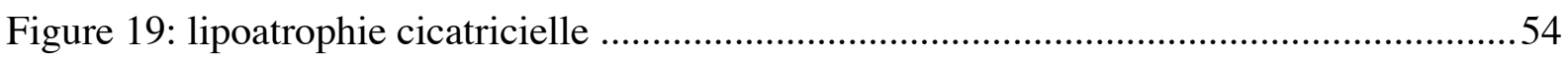

Figure 20: Répartition en pourcentage des grades de la dégénérescence graisseuse du 1/3 antérieur du muscle MF à 3 mois post opératoire ..........................................................6 60

Figure 21: Répartition en pourcentage des grades de la dégénérescence graisseuse du 1/3 antérieur du muscle MF à 12 mois post opératoire ......................................................6 60

Figure 22: Répartition en pourcentage des grades de la dégénérescence graisseuse du muscle

TFL à 3 mois post opératoire

Figure 23: Répartition en pourcentage des grades de la dégénérescence graisseuse du muscle

TFL à 12 mois post opératoire .62

Figure 24: résultats comparés de plusieurs cotations algo-fonctionnelle de hanche (d'après $\mathrm{J}$.

Witvoet, (81))

Figure 25: Comparaison des scores cliniques avec la littérature récente $(6,8,56,77,118,119) 73$

Figure 26: Comparaison des résultats paracliniques avec la littérature récente

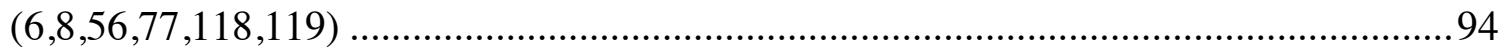

Figure 27:Comparaison des taux de complications dans la littérature récente

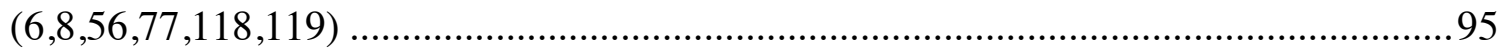

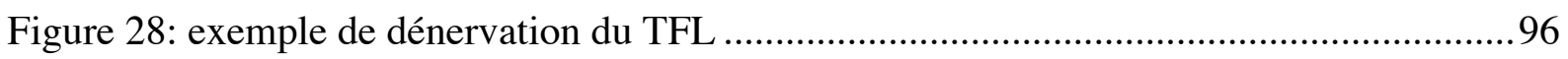




\section{Glossaire des termes, acronymes et abréviations utilisées \\ dans le manuscrit}

BIF : bloc d'analgésie ilio facial.

CHU : Centre Hospitalier Universitaire.

DG : dégénérescence graisseuse

EIAS : épine iliaque antéro supérieure

FOV : (Field of View), Champs de Vue.

FSE : Fat Spin Echo; dénomination utilisée par la Société General Electric Healthcare (Milwaukee/Wisconsin/USA).

Technique de séquence IRM associant la méthode écho de gradient et écho de spin pour une acquisition plus rapide. Cette technique appliquée à haut champ permet d'éviter certains artefacts dus aux spins mobiles.

GT : grand trochanter

HAS : Haute Autorité de Santé.

IDEAL : dénomination propre à la Société General Electric Healthcare (Milwaukee/Wisconsin/USA) ; séquence IRM particulière de séparation du signal de l'eau et de la graisse avec asymétrie d'écho puis de reconstruction de l'image par un algorithme d'estimation de congruence de carrés ou algorythme de Dixon («Iterative Decomposition of water and fat with Echo Asymmetry and Least - squares estimation »).

IMC : indice de masse corporelle, exprimé en $\mathrm{Kg} / \mathrm{m}^{2}$.

IRM : imagerie par résonnance magnétique.

MF : muscle moyen fessier.

PF : muscle petit fessier

Pondération T1 : pondération (IRM) dite «anatomique », utilisant un temps de répétition court $(\approx 10-20 \mathrm{msec})$ et un temps d'écho court $(\approx 400-600 \mathrm{msec})$.

Les liquides apparaissent en hyposignal. 
Pondération T2 : pondération (IRM) dite «tissulaire », utilisant un temps de répétition long (>2000 msec) et un temps d'écho long (>80 ms).

L'eau et l'oedème apparaissent en hypersignal.

ROI : region of interest ; zone ou région d'une image, délimitée par un contour, sur lequel un paramètre particulier est évalué. La sélection d'une ROI est faite manuellement par l'utilisateur ou par certains algorithmes d'extraction automatique.

Score ASA : score de l'American Society of Anesthesiology ; score de la société américaine d'anesthésie.

Score HSS : Harris Hip Score ; cotation fonctionnelle de hanche de Harris.

Score PMA : évaluation chiffrée de Postel, Merle d'Aubigné.

STIR : Short Time Inversion Recovery.

Les séquences (IRM) STIR ont pour but d'annuler le signal de la graisse.

TDM : tomodensitométrie

TE : temps d'écho, exprimé en msec.

Paramètre principal caractérisant une séquence IRM (avec le TR). Durée entre le sommet de l'impulsion magnétique d'excitation et la moitié du temps de lecture.

TFL : muscle tenseur du fascia lata.

TR : temps de répétition, exprimé en msec.

Paramètre principal caractérisant une séquence IRM (avec le TE). Durée au bout de laquelle on reproduit la séquence.

UH : Unité Hounsfield ; unité de mesure de la densité au scanner ou tomodensitométrie.

VALMI : voie d'abord antéro latérale mini invasive. 


\section{INTRODUCTION}

Lors d'une arthroplastie totale de hanche, l'intérêt et le bénéfice des voies d'abord miniinvasives restent encore discutés (1-8). Seules les voies antéro latérale mini-invasive (VALMI) et antérieure, ne comportent aucune section musculaire (9-11). Elles sont dites interstitielles et progressent soit en avant, soit en arrière du muscle tenseur du fascia lata (figure 1). Ces abords mini-invasifs respectent les structures postérieures (capsule et muscles rotateurs externes), essentielles à la stabilité de l'arthroplastie (12-14) et les muscles abducteurs, garants d'une récupération fonctionnelle rapide (8). Ce travail présente les résultats d'un chirurgien expérimenté qui utilise en pratique courante un abord antéro latéral mini invasif, initialement décrit par Bertin KC. et Röttinger H. en 2004 et $2010(15,16)$.

Des études électromyographiques, d'analyse de la marche ou le dosage des marqueurs musculaires sanguins, permettent une approche des lésions musculaires induites par la chirurgie $(6,8,17-21)$. Cependant, ces travaux ne permettent qu'une évaluation indirecte. Enfin, les travaux de recherche de dissections cadavériques n'offrent qu'une approche descriptive des lésions traumatiques et n'évaluent pas la notion évolutive de capacité régénérative ou de compensation musculaire $(2,4)$.

Le but de l'étude était l'évaluation objective du respect du capital musculaire locorégional, par une étude clinique et IRM, à 3 et 12 mois post opératoire, en comparaison au côté non opéré. Notre hypothèse était que l'utilisation d'un abord chirurgical antéro latéral mini invasif réputé atraumatique, pouvait induire des lésions musculaires infra cliniques (contusion ou étirement liés aux écarteurs, dénervation ... ), dépistables à l'IRM. Nous avons de plus étudié la capacité régénérative des muscles MF et TFL entre ces deux bornes temporelles ainsi que l'existence d'une éventuelle hypertrophie compensatrice. 

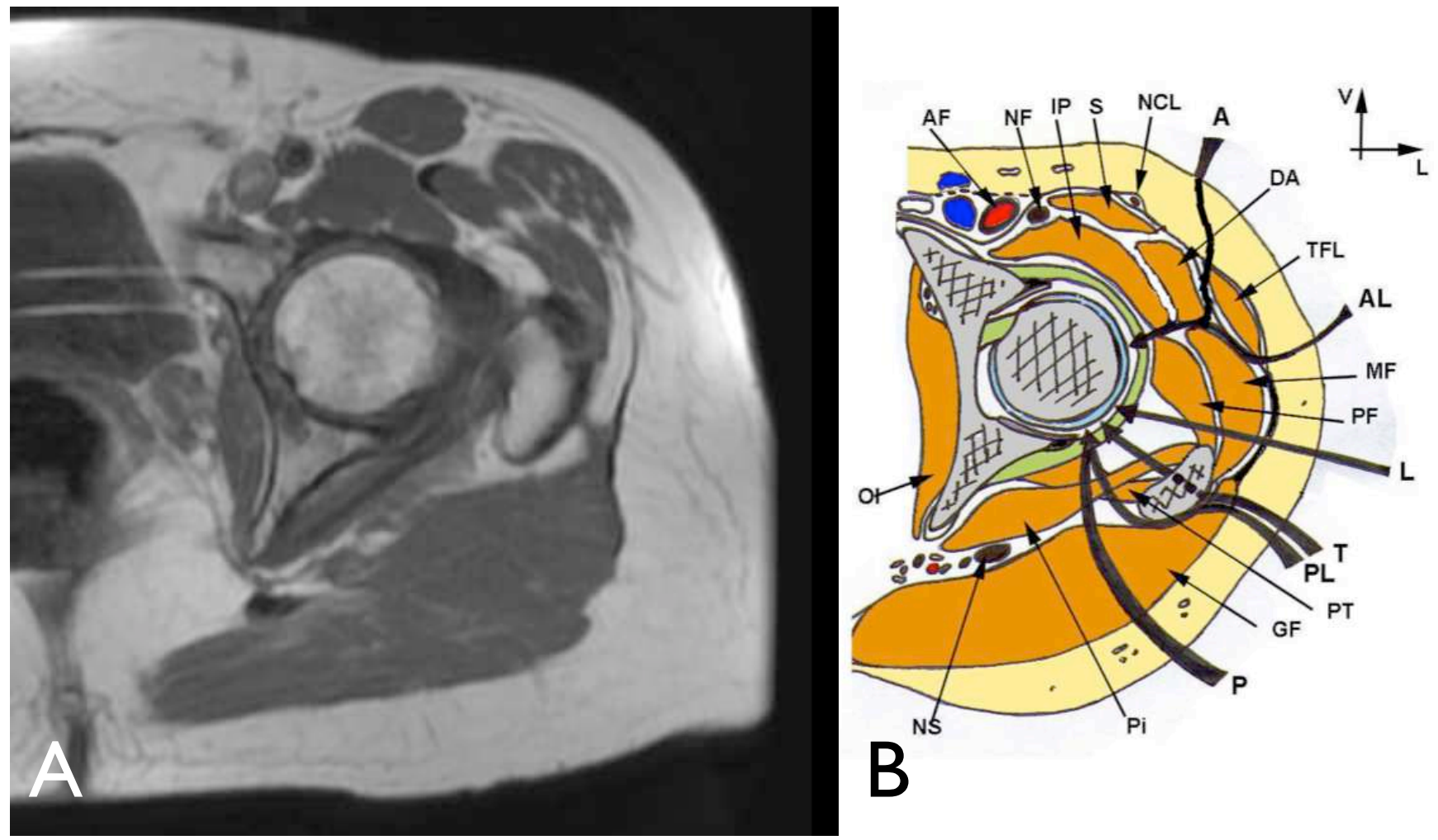

Figure 1: Coupes transversales de hanche.

A: séquence IRM inPhase T1 IDEAL FSE;

B: schéma anatomique (d'après Pr Duparc F.) (9).

$A F$ : artère fémorale ; NF : nerfs fémoral; IP : muscle ilio-psoas ; $S$ : muscle sartorius ; NCL : Nerfs cutanée latéral ; $\boldsymbol{A}$ : Voie d'abord antérieure ; DA : muscle droit antérieur de la cuisse; TFL : muscle tenseur du fascia lata ; AL : voie d'abord antéro latérale; MF : muscle moyen fessier ; PF : muscle petit fessier ; $\mathbf{L}$ : voie latérale (transglutéale); $\boldsymbol{T}$ : voie par trochantérotomie ; PL : voie d'abord postéro latérale ; PT : muscles pelvitrochanteriens: ; GF : muscle grand fessier ; $\boldsymbol{P}$ : voie d'abord postérieure ; Pi : muscle piriforme; NS : nerfs sciatique ; Oi : muscle obturateur interne. 


\section{GENERALITES}

\subsection{Rappel anatomique des muscles abducteurs de hanche}

L'articulation coxo-fémorale est une énarthrose profonde et stable. Elle est entourée de structures vasculo-nerveuses et de muscles puissants : il existe des groupes musculaires antérieur, postérieur, médial et latéral.

Situé latéralement, le muscle moyen fessier est essentiel à l'abduction de hanche. Il s'insère par des fibres charnues dans la fosse iliaque externe, entre les deux lignes glutéales (antérieure et postérieure) et jusqu'à la crête iliaque où il détermine le tubercule fessier. Il se dirige en bas et en dehors et se termine par un épais tendon sur la face externe du grand trochanter (figure 2). Robertson et al (22) ont décrit en 2008, à partir d'une étude cadavérique et d'un logiciel de navigation tridimensionnelle, une insertion terminale selon deux sites distincts à la face latérale $\left(438.0 \mathrm{~mm}^{2} ; \mathrm{SD}, 57.7 \mathrm{~mm}^{2}\right)$ et postéro-supérieure $\left(196.5 \mathrm{~mm}^{2}\right.$; SD, $\left.48.4 \mathrm{~mm}^{2}\right)$ du grand trochanter. Flack et al (23) ont réalisé en 2012, une revue de la littérature publiée sur l'anatomie fonctionnelle et descriptive des muscles abducteurs de la hanche. Cette étude de bibibliographie bien menée sur le plan méthodologique, analysait 1207 articles scientifiques pour ne retenir que 43 articles scientifiques importants et 9 livres d'anatomie. A partir de cette littérature les auteurs (23) ont étudié et comparé les sites d'insertion proximal et distal des muscles petit, moyen fessier et du TFL ainsi que plusieurs autres paramètres (innervation, volume, surface de section à la coupe longueur, orientation des fibres, fonction...). A titre indicatif, nous utilisons dans ce travail deux schémas illustrant les variations d'insertions musculo tendineuses décrites par cette revue de littérature (fig $2 \mathrm{C}$ et 3 ).

Le moyen fessier constitue le muscle fondamental pour l'équilibre horizontal du bassin lors de l'appui uni-podal (24). Plusieurs études biomécaniques et fonctionnelles distinguent différents rôles moteurs selon la composante antérieure, moyenne ou postérieure des fibres musculaires. La combinaison de l'orientation verticale des fibres du 1/3 moyen du MF (25$27)$, d'un grand bras de levier dans le plan frontal $(28,29)$ et d'une large surface de section musculaire (30) suggère qu'il possède, en position anatomique, un fort pouvoir moteur d'abduction et un rôle important dans la stabilité du pelvis en position statique. En revanche, l'orientation de ces fibres postérieures, leur petit bras de levier dans le plan frontal et leur surface de section musculaire moins importante $(28,29)$ auraient un rôle de stabilisateur de la tête fémorale $(25,26)$. Aussi en 2013, Semciw et al (30) ont montré que les parties moyenne et postérieure du moyen fessier présentaient un rôle synchronisé au cours de la marche. Les 
fibres antérieures du moyen fessier auraient quant à elles, une double composante. Leur orientation verticale $(25-27)$, le grand bras de levier $(28,29)$ et leur surface musculaire participeraient avec les fibres moyennes, à l'équilibre horizontale du bassin pendant la phase d'appui de la marche (26). Une analyse EMG (30) évoque également un rôle synergique durant la phase tardive d'appui (propulsion), des fibres antérieures dans le contrôle de l'extension (31) de la hanche ou dans le contrôle de la rotation $(25,26,28)$ du bassin dans le plan transversal.

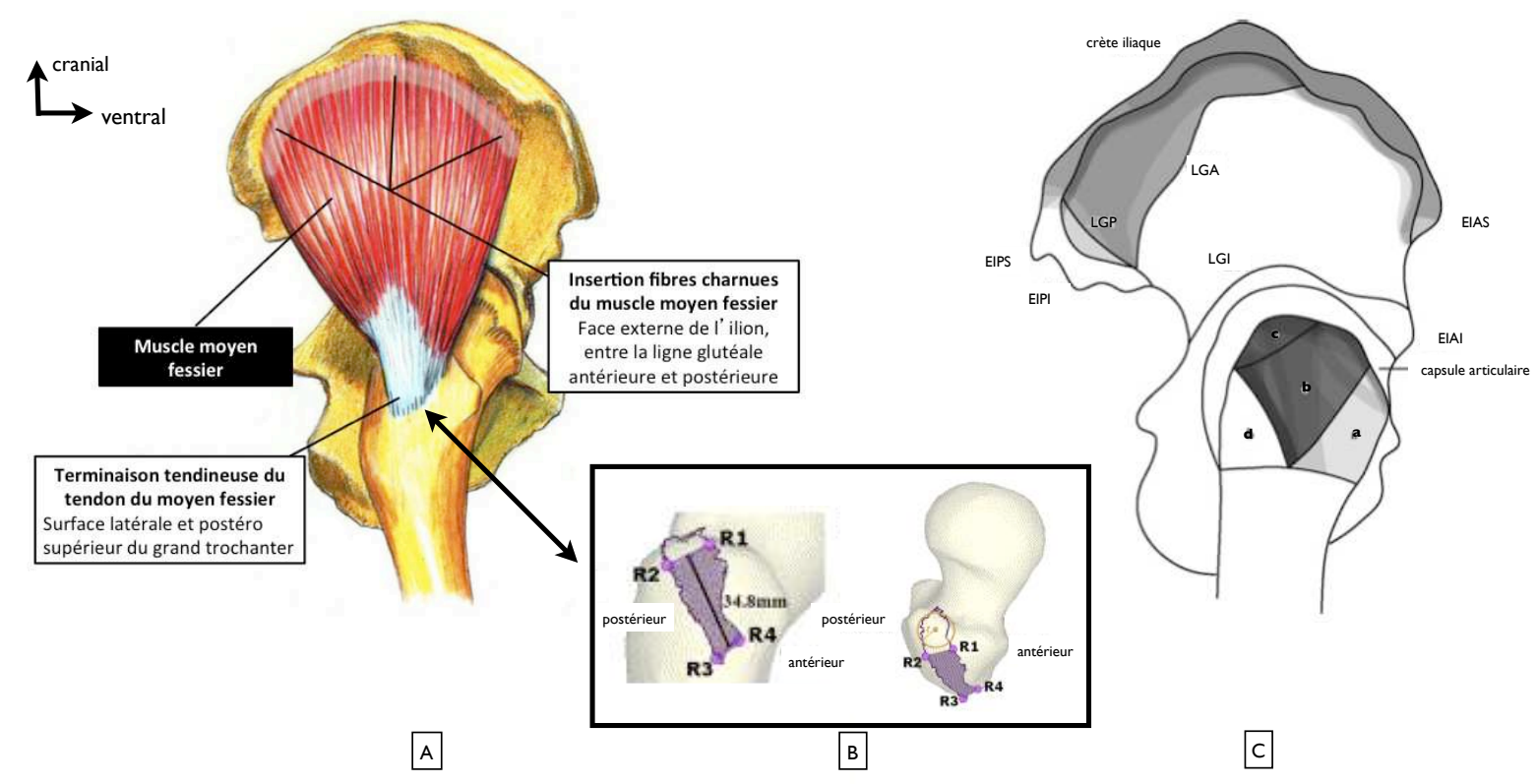

Figure 2: Schéma représentant le muscle moyen fessier.

A : vue latérale; B : description de son insertion distale tendineuse décrite par Robertson et al (22); C: description de la variation des sites d'insertion proximal et distal du moyen fessier selon une revue de littérature publiée par Flack et al. en 2012 (23). Plus la zone est sombre, plus elle est citée dans la littérature scientifique. EIAS : épine iliaque antéro supérieure; EIAI: épine iliaque antéro inférieure; $a$ : facette antérieure ; $b$ : facette latérale; $c$ : facette postéro supérieure; $d$ : facette postérieure du GT; LGI : ligne glutéale inférieure; LGA :ligne glutéale antérieure; LGP :ligne glutéale postérieure; EIPI :épine iliaque postéro inférieure ; EIPS :épine iliaque postéro supérieure) (d'après Flack et al. (23)).

Cette stabilité est également permise grâce à d'autres muscles :

- Le petit fessier, situé au plan profond de la fesse, s'insère sur la fosse iliaque externe en dessous de la ligne glutéale antérieure et au dessus de la ligne glutéale inférieure. Son trajet descend oblique en bas et en dehors, au dessus de l'articulation coxofémorale. Il se termine à la face antérieure du grand trochanter. En plus de son rôle moteur principal d'abduction de la hanche, il participe également à la rotation interne. 
En périphérie (plan superficiel) le tractus ilio-tibial y participe également : il forme avec le TFL et les fibres superficielles du grand fessier, le deltoïde fessier (32) qui contrôle et décélère l'adduction de la cuisse pendant la course à pied, par exemple $(24,33,34)$.

- Le Tenseur du fascia lata est un muscle fusiforme, bi articulaire, depuis l'épine iliaque antéro supérieure au bord antérieur du fascia glutéal. Il participe à l'abduction / flexion de hanche et à la rotation interne du genou. Des études cadavériques et EMG ont mis en évidence que le TFL jouait un rôle de fléchisseur de hanche important durant la phase oscillante de la marche ainsi que d'abducteur/ stabilisateur durant la phase d'appui complet de la marche (26).

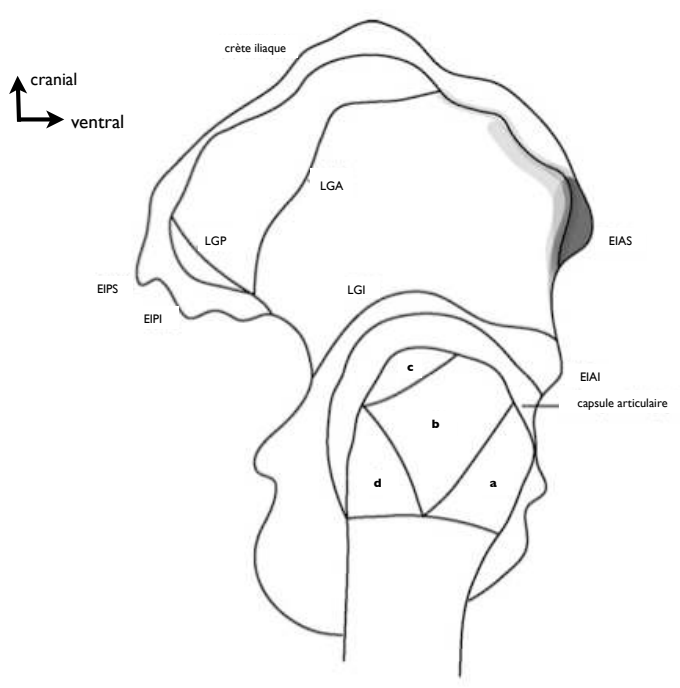

Figure 3: Description anatomique du site d'insertion proximal du TFL

Description de la variation des sites d'insertion proximale et distale du moyen fessier selon une revue de littérature décrite par Flack et al. en 2012 (23). Plus la zone est sombre, plus elle est citée dans la littérature scientifique. EIAS: épine iliaque antéro supérieure; EIAI: épine iliaque antéro inférieure; a: facette antérieure ; $b$ : facette latérale ; $c$ : facette postéro supérieure ; $d$ : facette postérieure du GT ; LGI : ligne glutéale inférieure; LGA :ligne glutéale antérieure; LGP :ligne glutéale postérieure; EIPI :épine iliaque postéro inférieure ; EIPS :épine iliaque postéro supérieure) (d'après Flack et al. (23)). 
- Plus accessoirement :

- Le muscle piriforme situé à la partie postérieure du plan profond de la fesse. Muscle pelvi-trochantérien, il s'insère à la face antérieure du sacrum de part et d'autre des $2^{\text {é et }} 3^{\text {é }}$ trous sacrés, sort du pelvis par la grande échancrure sciatique et passe en arrière et au dessus de l'articulation coxo-fémorale. Il se termine à la face supérieure du GT. Il est abducteur et rotateur externe de hanche.

- Le muscle sartorius, en avant, superficiel et bi articulaire. Il appartient aux groupes musculaires de la cuisse. Il est oblique en bas et en dedans, depuis l'épine iliaque antéro supérieure jusqu'à la patte d'oie, sur la tubérosité médiale du tibia. Il participe à la flexion/abduction/rotation latérale de hanche et flexion/rotation médiale du genou.

Ces muscles abducteurs contribuent à de nombreuses actions motrices, incluant la stabilité (transversale et frontale) du pelvis durant la marche, à l'abduction et à la rotation de l'articulation coxo-fémorale (23).

\subsection{Innervation des muscles abducteurs de hanche}

Tous les muscles abducteurs de l'articulation coxo-fémorale sont innervés par les branches collatérales du plexus sacral, sauf le muscle sartorius qui est innervé par le nerf fémoral (branche du plexus lombal). Le plexus sacral donne une branche terminale : le nerf sciatique, et des branches collatérales innervant les muscles de la région glutéale. (Figure 4) :

- Le nerf glutéal supérieur accompagne l'artère glutéale supérieure dans le canal supra-piriformien. Deux principaux types de ramifications nerveuses sont décrites dans la littérature $(35,36)$ :

- un modèle de ramification en troncs ou branches nerveuses cheminant transversalement pour se placer dans le plan situé entre le moyen et le petit fessier qu'il innerve avant de se terminer dans le tenseur du fascia lata. Cette première description anatomique semble être la plus communément admise et 
décrite (34,37-48). Il existerait deux principales ramifications : 1 branche craniale issue des racines L4-S1 (41) et 1 branche caudale issue des racines S1 et S2 (41). Plus récemment, en 2013, Apaydin et al (36) décrivaient 3 variations principales : type A (48.5\%): une seule branche destinée au moyen fessier et une branche commune destinée au petit fessier + TFL; type B (29.2\%): une branche commune pour le moyen et petit fessier et un autre tronc commun pour le moyen fessier + TFL ; type C (16.7\%): une branche commune pour le moyen + petit fessier et une seule branche pour le tenseur du fascia lata.

- un modèle composé de ramifications nerveuses multiples dites en «spray» avec un ou plusieurs courts rameaux nerveux innervant le petit et moyen fessier, et une ou plusieurs longues branches innervant le tenseur du fascia lata $(36,49)$.

Le non respect de ce nerf glutéal supérieur durant une intervention de chirurgie arthroplastique de hanche préjuge d'un mauvais résultat fonctionnel. De nombreuses études ont ainsi analysé son risque lésionnel durant les abords chirurgicaux et les différents temps opératoires ainsi que des solutions ou « zones de sécurité » $(35,36,39,45,47-53)$.

- Le nerf du muscle piriforme se jette dans le muscle à hauteur de la grande incisure sciatique.

- Le nerf glutéal inférieur accompagne le nerf sciatique dans le canal infra piriformien vers le muscle grand fessier.

- Les autres branches nerveuses collatérales sont destinées aux quatre autres muscles pelvi trochantériens (obturateur interne, jumeaux, carré fémoral). 

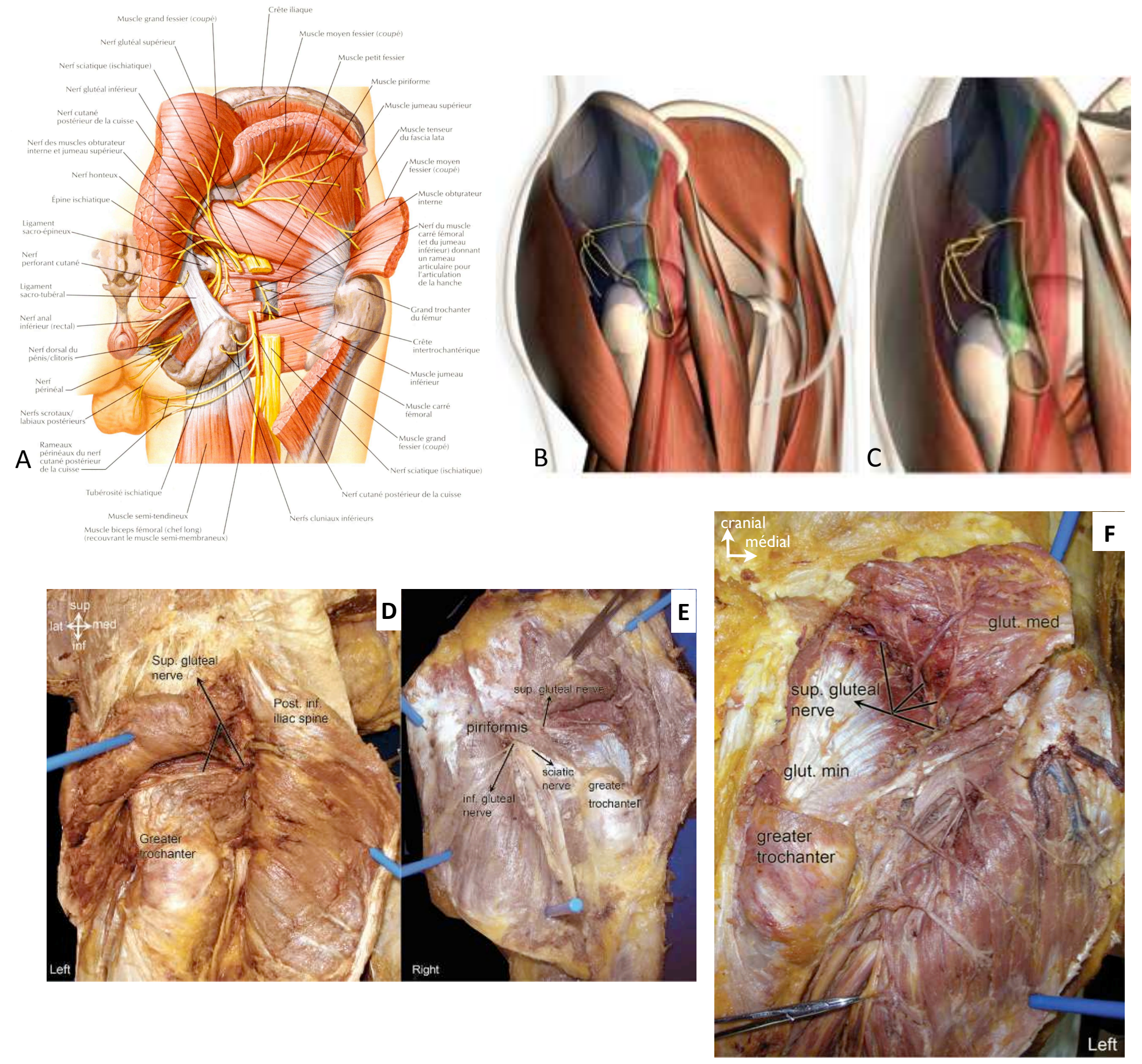

Figure 4 : Schémas anatomiques de l'innervation glutéale.

A : hanche droite, vue postérieure (d'après Netter F.) (54); B : hanche droite, vue latérale de la boucle de connexion nerveuse glutéale supérieure innervant le TFL (en jaune) décrit par Ince A. et al (49) (muscle moyen fessier en bleu, m. petit fessier en vert, m. TFL en rouge) ; $\mathbf{C}$ : hanche droite, vue antéro latérale (d'après Ince A. et al) (49) ; D : hanche gauche, vue latérale du modèle de distribution nerveuse en « tronc » d'après Apaydin et al (36); E : hanche droite, vue latérale (d'après Apaydin et al ) (37); F : hanche gauche, vue latérale du modèle de distribution nerveuse en « spray » d'après Apaydin et al. (36). 


\subsection{Risques lésionnels théoriques dans la VALMI}

L'absence de section musculaire durant l'intervention, ne garantit pas l'absence de lésions musculaires ou nerveuses induites par l'abord. Il peut s'agir de lésions musculaires du PF / MF et TFL à type de contusion pouvant être causées par une tension continue des écarteurs $(52,55)$ ou par un traumatisme direct des râpes fémorales $(56,57)$. Ces lésions peuvent également être de type nerveuses (58) par lésion du nerf glutéal supérieur: soit en proximal dans sa portion cheminant entre les muscles moyen et petit fessier, soit de manière plus distale, dans sa branche terminale destinée au TFL.

Nous énumérerons ces lésions potentielles à la lumière d'une expérience chirurgicale, en suivant les principaux temps opératoires :

- Abord chirurgical : la branche terminale du nerf glutéal supérieur peut être lésée à la partie proximale de l'interstice entre le corps musculaire du MF en arrière et du TFL en avant. Cette branche est très souvent visualisée (environs $50 \%$ des cas selon notre expérience). Nous pensons prévenir ce risque en réalisant une dissection minutieuse de l'interstice et non par digitoclasie (figure 5).
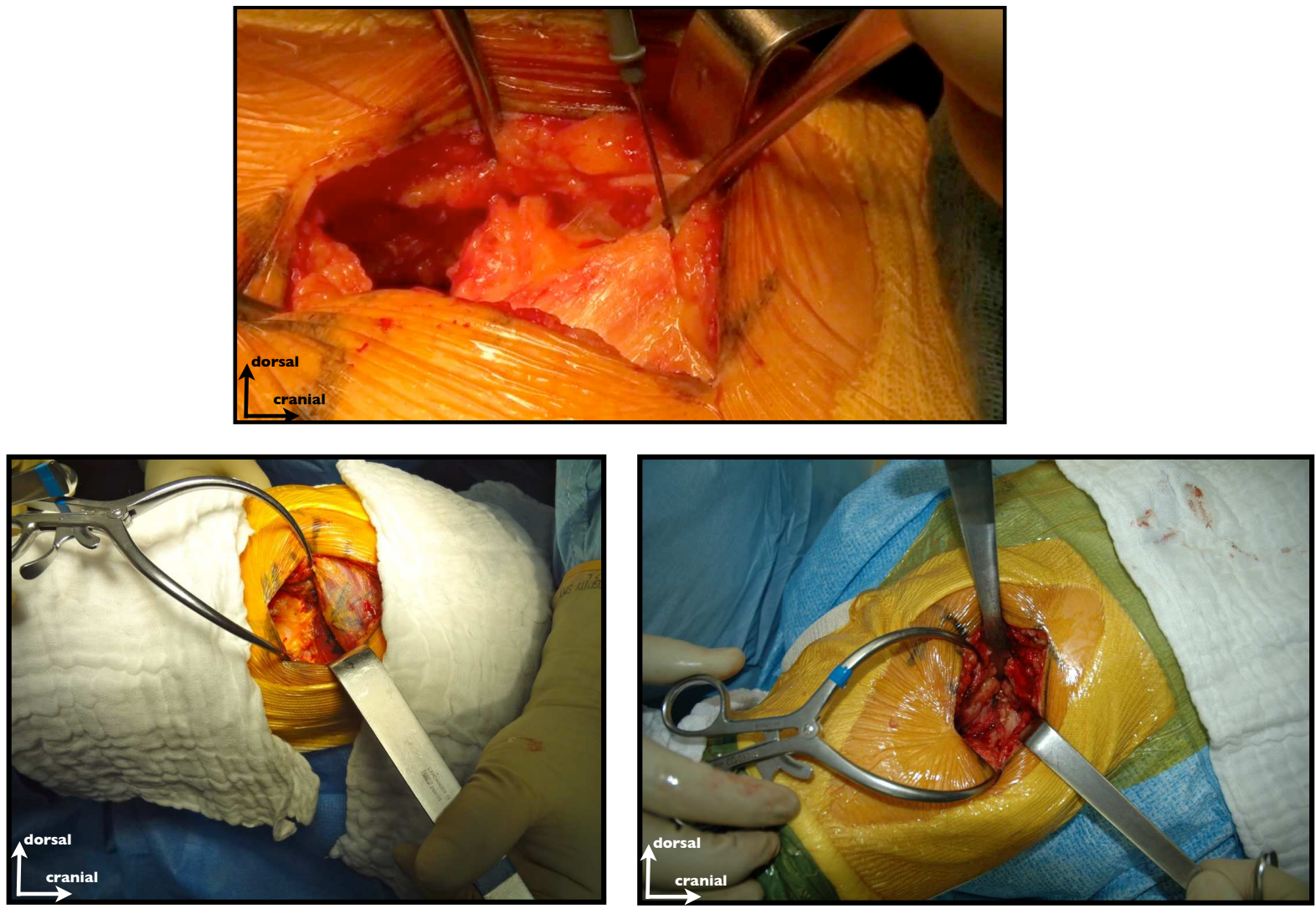

Figure 5: Risques lésionnels durant l'abord 
- Temps acétabulaire : nous pensons que les risques lésionnels sont faibles, tant en ce qui concerne la contusion musculaire que le risque neurologique (figure 6).

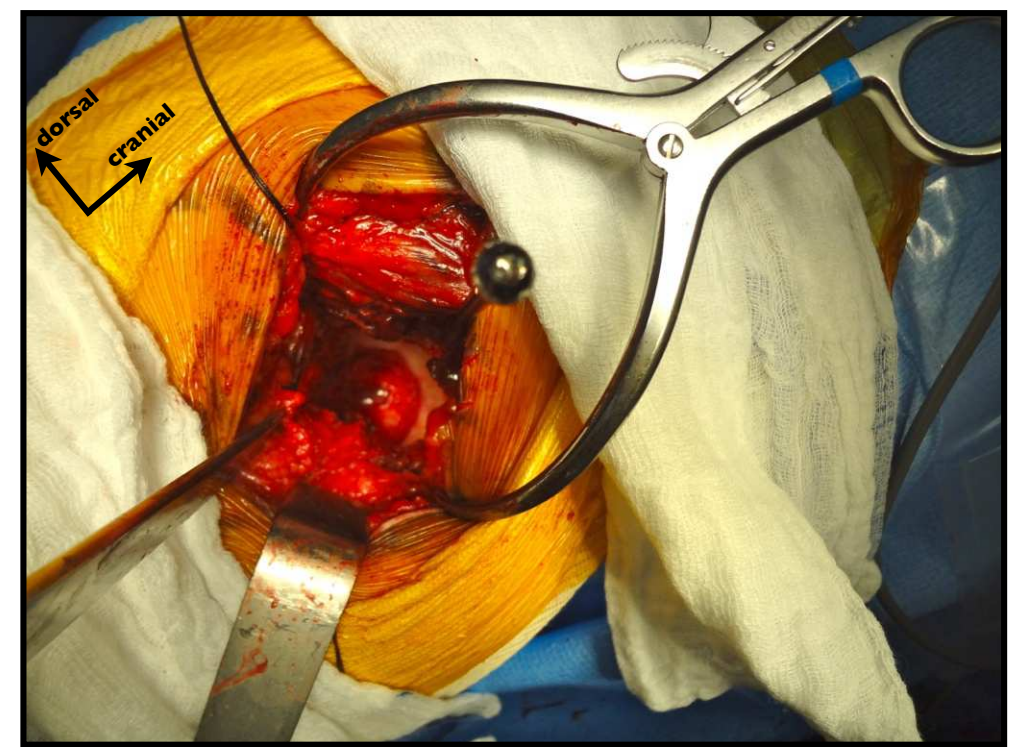

Figure 6: Risques lésionnels durant le temps acétabulaire

- Temps fémoral : le risque est important pour le muscle moyen fessier. L'écarteur de Hohman situé en arrière, à la face profonde du PF et refoulant le MF, ainsi que les râpes fémorales peuvent léser les fibres antérieures du muscle MF (56,57). Ces instruments peuvent également comprimer ou étirer les branches terminales du nerf glutéal supérieur. L'élévateur fémoral antérieur présente peu de risque pour le corps musculaire du TFL en avant (figure 7).

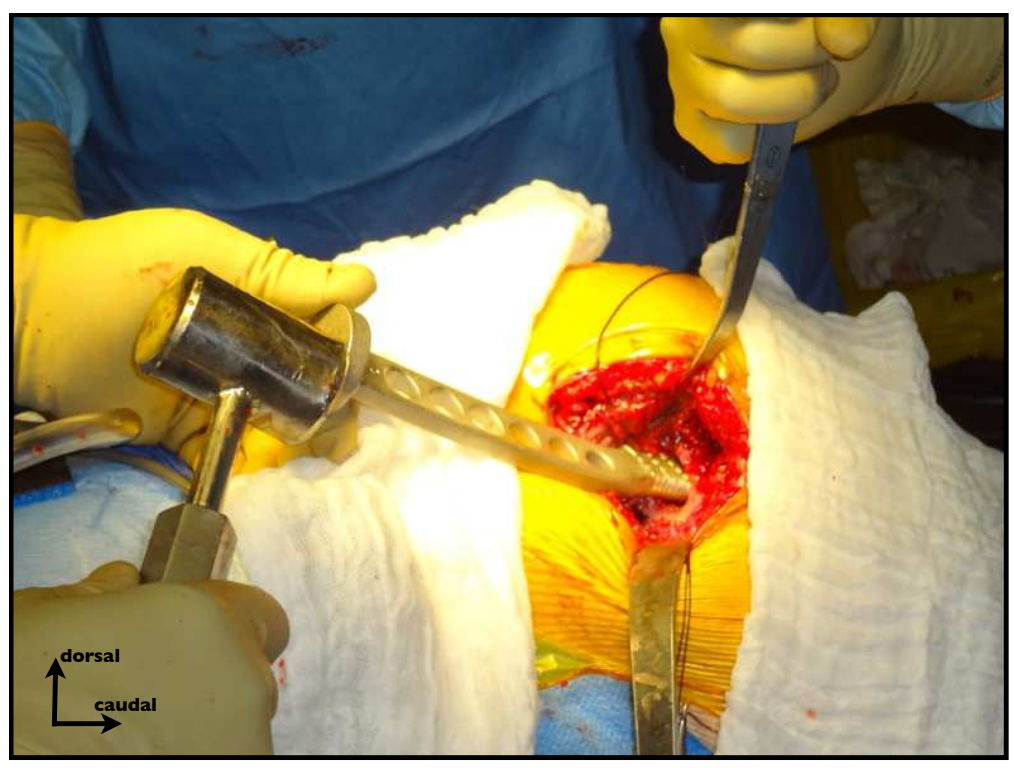

Figure 7: Risques lésionnels durant le temps fémoral 
En conclusion, nous pensons que ces lésions peuvent se distinguer principalement en deux types :

- Contusion du 1/3 antérieur des fibres musculaires du moyen fessier.

- Dénervation musculaire du TFL par compression/étirement ou section de la branche terminale du nerf glutéal supérieur.

Une étude de dissections de sujets anatomiques réalisée en 2010 par Oldenrijk et al (53) a comparé 5 voies d'abord de hanche :

- Concernant l'existence de lésions musculaires : ils n'ont pu mettre en évidence de diminution lésionnelle significative des muscles fessiers (petit, moyen et grand), carré fémoral, droit antérieur et TFL pour aucun des abords mini invasifs testés (antérieure, antéro-latérale, postérieure et double abord) comparativement à la voie transglutéale. Pour la voie antéro-latérale mini invasive, les auteurs exposent une surface lésionnelle médiane de $18 \%[\mathrm{mini}=6 \% ; \operatorname{maxi}=27 \%]$ par coupe de section transversale étudiée du moyen fessier.

- Concernant les lésions de la branche du nerf glutéal supérieur destiné au TFL, les auteurs exposent un risque de section survenue dans 3 cas sur les 6 hanches opérées et pouvant théoriquement entrainer une lésion de dénervation du muscle. La fréquence de cette atteinte lésionnelle nous apparaît importante et justifie par précaution une dissection minutieuse de cette zone.

Si l'on considère l'innervation du TFL avec l'existence d'une boucle de connexion nerveuse glutéale (figure 4), comme l'ont mis en évidence Ince et al (49) dans une étude cadavérique dans 10 cas sur 20 hanches disséquées. Une atteinte de la branche terminale craniale du nerf glutéal supérieur à la partie proximale de l'interstice MF/TFL, entrainerait une dénervation seulement partielle (53). Outre le risque de section nerveuse, il existe un risque de lésions nerveuses par compression / étirement: Noble et al (52) ont mis en évidence en 2007, une augmentation des forces de traction / compression trois fois supérieures sur les tissus mous environnants dans le cadre des abords mini invasifs, comparativement aux abords conventionnels. 
Notre série 


\section{MATERIEL \& METHODE}

\subsection{Objectifs de l'étude}

Le but de cette étude est l'évaluation objective du respect du capital musculaire locorégional du moyen fessier et du TFL, après une chirurgie de remplacement arthroplastique bipolaire de hanche, par voie d'abord antéro latérale mini invasive.

L'objectif secondaire est l'évaluation de la capacité régénérative ou d'adaptation des muscles MF et TFL entre le $3^{\text {éme }}$ et le $12^{\text {éme }}$ mois post opératoire.

\subsection{Type d'étude}

Il s'agit d'une étude descriptive, prospective sur une série continue de patients opérés entre le $1^{\text {er }}$ avril 2011 et le 6 décembre 2011. Cette étude mono centrique, mono-opérateur s'est déroulée au sein du Groupe Hospitalier du Havre, Jacques Monod (29 Av Pierre Mendes France, 76290 Montivilliers, France).

Tous les patients ont été opérés par le même chirurgien. L'ensemble des données cliniques et IRM étaient recueillies par un examinateur unique et indépendant de l'opérateur.

\subsection{Population étudiée}

Les patients étaient sélectionnés lors de la consultation d'orthopédie du Dr Matsoukis, au cours de laquelle une indication chirurgicale de remplacement coxo-fémoral arthroplastique de première intention était posée.

Le patient recevait une information claire et les modalités du protocole d'étude lui étaient exposées lors de la consultation préopératoire afin de recueillir son consentement oral. 


\subsection{Critères d'inclusion et critères d'exclusion}

Les critères d'inclusion tenaient compte de la voie d'abord chirurgicale, et de l'absence d'antécédent d'abord chirurgical sur les deux hanches. L'accord du patient devait être obtenu pour la réalisation du protocole d'étude clinique et para clinique. Les patients opérés devaient accepter le suivi médical avec un recul minimum de 1 an post-opératoire et la réalisation des IRM à 3 et 12 mois post-opératoire.

L'existence d'une obésité morbide constituait un critère d'exclusion pour ce type de voie d'abord. Les autres critères d'exclusion veillaient à éliminer les perturbations pré-opératoires des paramètres étudiés et les pathologies générales intercurrentes. Ils comprenaient également l'ensemble des contre-indications à la réalisation d'une imagerie par résonance magnétique.

Les critères d'inclusion et d'exclusion sont résumés dans le tableau 1.

Tableau 1: Critères d'inclusion et d'exclusion

\begin{tabular}{|c|c|}
\hline Critères d'inclusion & Critères d'exclusion \\
\hline PTH de première intention & $\begin{array}{l}\text { Antécédent d'autre abord chirurgical sur le } \\
\text { côté de la hanche opérée pour l'étude. }\end{array}$ \\
\hline $\begin{array}{l}\text { Voie d'abord antéro latérale mini invasive de } \\
\text { Röttinger. }\end{array}$ & $\begin{array}{l}\text { Etiologie pré opératoire traumatique ou } \\
\text { dysplasique }>\text { Crow type } 1(59) .\end{array}$ \\
\hline $\begin{array}{l}\text { Hanche controlatérale vierge de tout } \\
\text { antécédent d'abord chirurgical }\end{array}$ & Pathologie neuromusculaire. \\
\hline Patient âgé de plus de 18 ans. & Trouble cognitif. \\
\hline \multirow[t]{3}{*}{ Consentement au protocole d'étude } & $\mathrm{IMC}>40 \mathrm{~kg} / \mathrm{m}^{2}$ \\
\hline & Contre-indications à l'IRM. \\
\hline & $\begin{array}{l}\text { Absence de réalisation des IRM à } 3 \text { et } 12 \\
\text { mois post opératoire. }\end{array}$ \\
\hline
\end{tabular}




\subsection{Effectif et perdus de vue}

L'effectif cible de cette étude était de 30 patients opérés et ayant réalisé les deux IRM post opératoires ( 3 et 12 mois post op). Au dernier recul, nous retrouvions 30 patients opérés mais seulement 26 patients ayant réalisé les deux IRM post opératoire. Quatre patients $(13,33 \%)$ n'ont pu obtenir l'imagerie du $12^{\text {éme }}$ mois. Deux patients nous ont avoué être claustrophobes et avoir mal supporté le premier examen et deux autres ont dû subir une intervention chirurgicale nécessitant leur sortie du protocole (1 pathologie cardiovasculaire grave et 1 fracture post traumatique du fémur controlatéral).

L'effectif total de la série était donc de 26 patients ( 15 femmes, 11 hommes), d'âge moyen 68 ans \pm 9.94 ans [40 ans -86 ans] avec $86,67 \%$ de patients revus (examen clinique + IRM) à 12 mois post opératoire.

\subsection{Méthode}

\subsubsection{Technique chirurgicale utilisée}

La voie d'abord antéro latérale mini invasive utilisée était celle décrite historiquement par Bertin KC. et Röttinger H. en 2004 et $2010(15,16)$. Certains points techniques de l'intervention ont été modifiés par rapport à la technique initialement décrite (60). 


\subsubsection{Installation du patient}

L'intervention était réalisée en décubitus latéral sur table ordinaire. Le membre inférieur était maintenu en abduction de quelques degrés par un appui en $U$ au niveau du genou et de la jambe. L'appui sacré était fixé en position haute dans le but de prévenir le risque de compression sciatique pendant la luxation postérieure du membre au cours du temps fémoral de l'intervention (Figure 8).
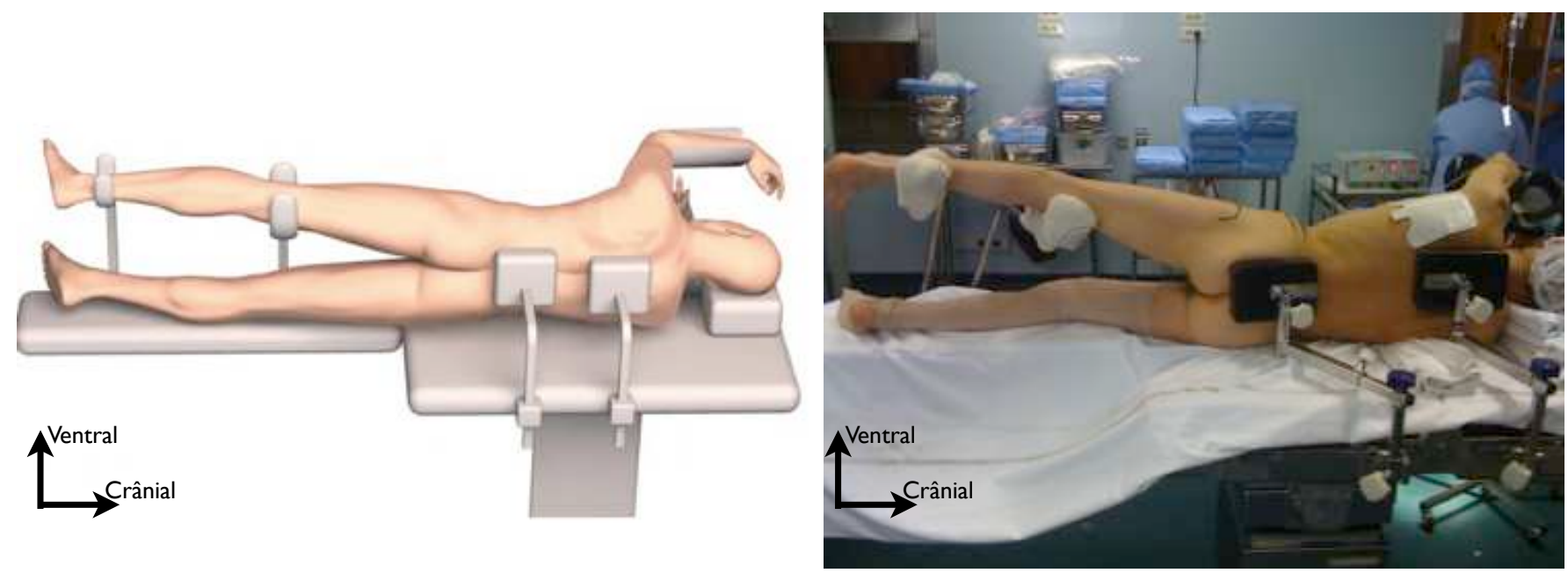

Figure 8: Installation du patient à opérer. Vue dorsale

\subsubsection{Anesthésie}

L'anesthésie était soit générale, soit rachidienne selon l'indication portée par l'anesthésiste en fonction des comorbidités présentes. L'essentiel était d'obtenir un relâchement musculaire optimal. Durant l'anesthésie générale, nous demandions à ce que le patient bénéficie d'une curarisation afin d'optimiser ce relâchement musculaire durant toute la durée de l'intervention chirurgicale. Aucune analgésie loco régionale complémentaire à type de bloc plexique lombal ou de bloc ilio facial n'était réalisée. 


\subsubsection{Voie d'abord}

Après détersion et champage chirurgical, la palpation des deux principaux reliefs osseux (EIAS et GT) était réalisée et le repérage de l'incision vérifié. Un champ collant antiseptique type Ioban ${ }^{\mathrm{TM}}$ ou Steri-Drape ${ }^{\mathrm{TM}}$ (société $3 \mathrm{M}^{\mathrm{TM}}$ Global Headquarters / Minnesota / USA) était utilisé. L’incision cutanée était située au bord antérieur du grand trochanter, centrée sur son sommet, dans l'axe du membre et mesurait de 7 à $10 \mathrm{~cm}$ selon le morphotype du patient. Röttinger $(15,16)$ a décrit une incision partant de l'EIAS au bord antérieur du grand trochanter. Nous l'avons modifiée dans le but de diminuer les risques de lésions cutanées et musculaires du bord antérieur du moyen fessier pendant l'intervention (Figure 9). Cette modification de l'incision et les bénéfices attendus furent secondairement décrit par Noble et al en 2012 (61). Devant un patient musclé ou obèse et en présence de difficultés d'exposition, l'incision était agrandie.
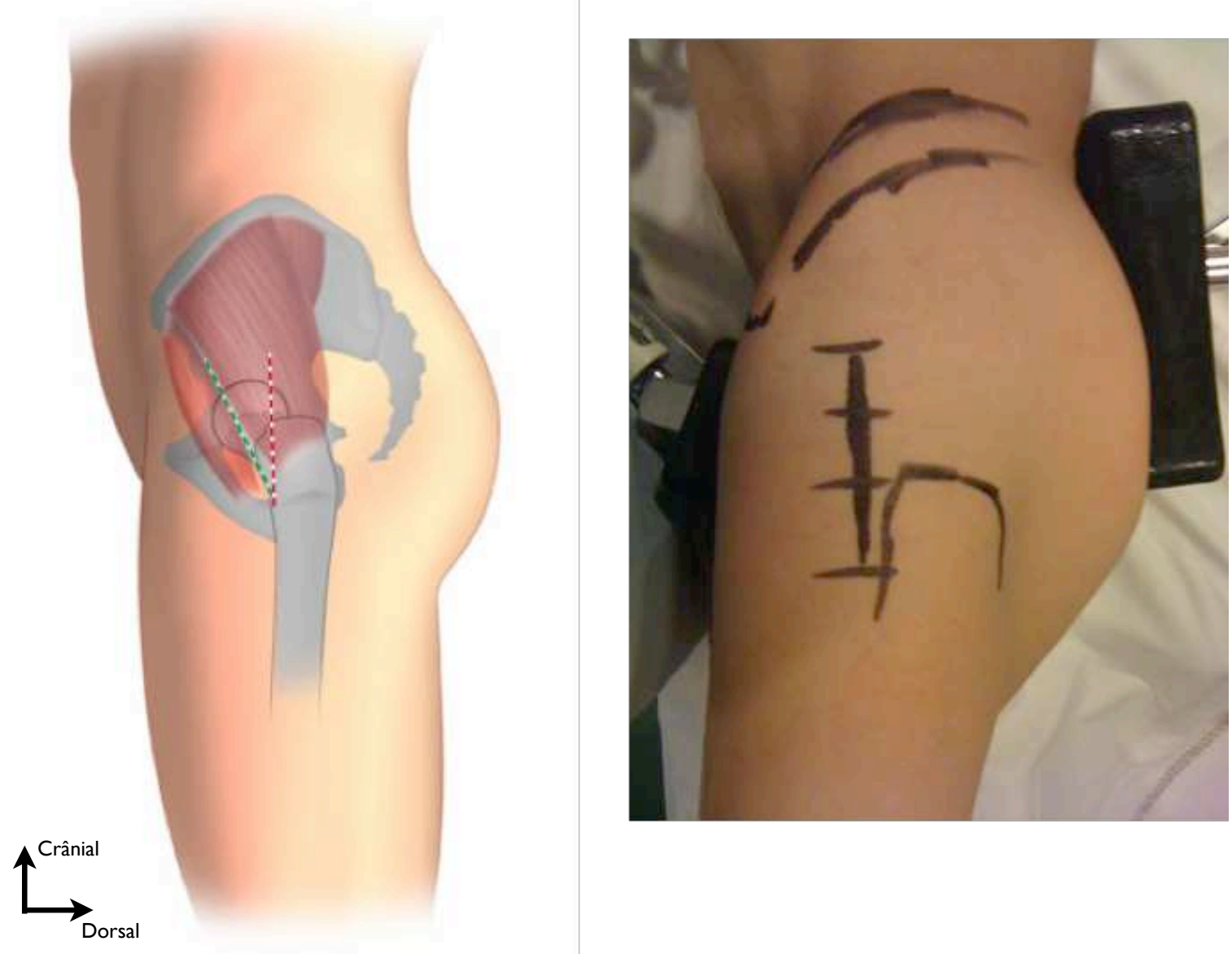

Figure 9: Dessins des voies d'abord et des repères osseux 
L'aponévrose était incisée au bord postérieur des fibres musculaires du tenseur du fascia lata, à l'union des fibres blanches du fascia glutéal, et généralement située à l'aplomb du grand trochanter. La dissection de l'interstice entre les corps musculaires du moyen fessier et du TFL commençait à la partie distale de l'incision, située entre l'insertion proximale du muscle vaste latéral, en avant, et le contingent antérieur des fibres distales du MF en arrière (Figure 10). Cette dissection de l'interstice était poursuivie de distale en proximale jusqu'au tendon réfléchi du muscle droit de la cuisse s'insérant dans le sillon supra acétabulaire au dessus du sourcil. Cette libération minutieuse de l'interstice avait pour but de faciliter l'exposition notamment lors du temps fémoral et le risque de lésion de la portion antérieure du moyen fessier par les écarteurs et les râpes, rapportés par Martin et al (56) ainsi que Zhang et al (57) dans près de $20 \%$ des cas. A la partie proximale de l'interstice, Ince et al (49) ont décrit la présence dans $50 \%$ des cas d'une branche terminale du nerf glutéal supérieur, innervant pour partie le TFL. Celle ci était respectée lorsqu'elle était visualisée. Le temps suivant consistait à exposer la capsule, avec l'arthrotomie, permettant l'exposition de la tête fémorale et du col. 

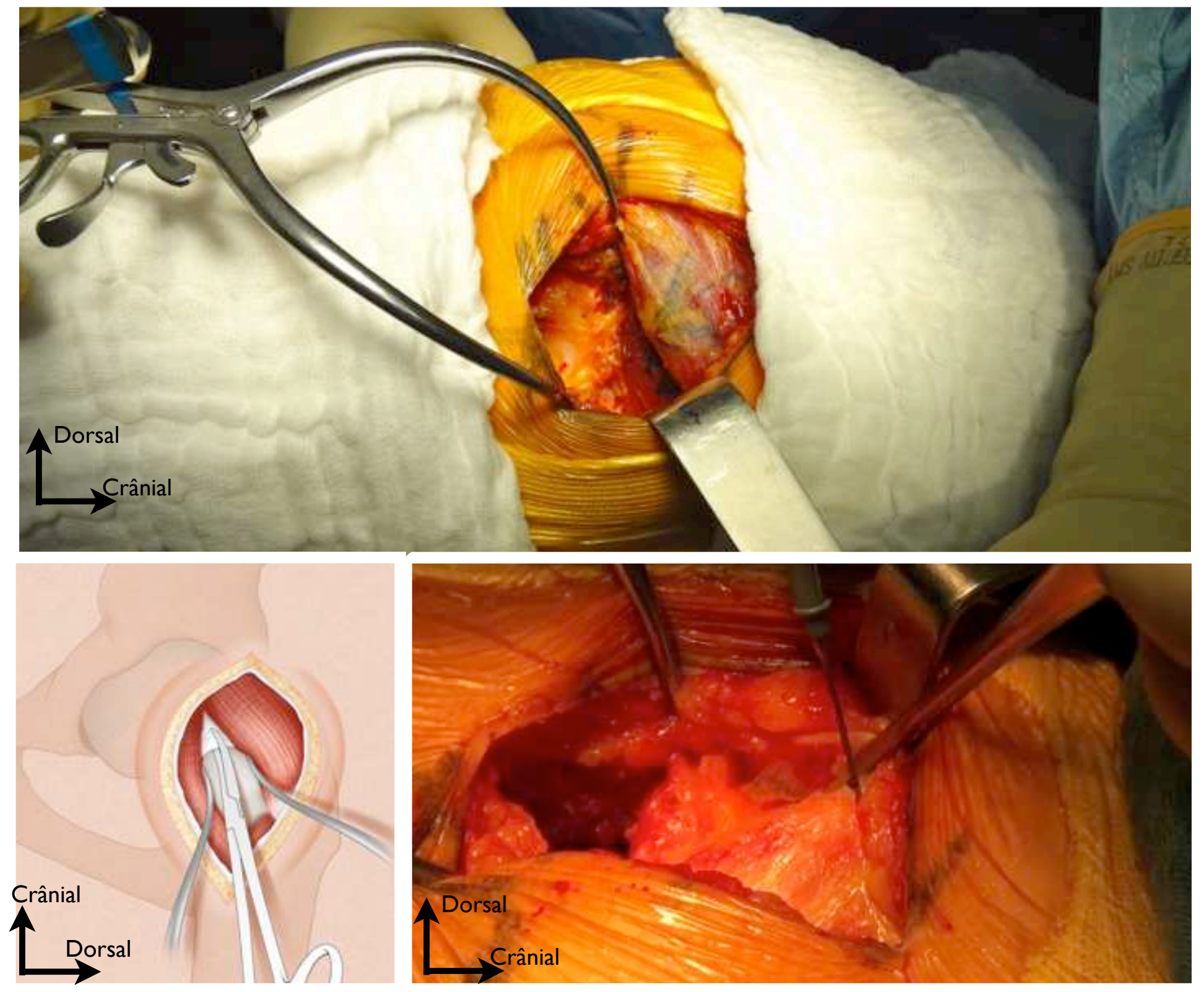

Figure 10: Voie d'abord. Dissection de l'interstice situé entre le muscle MF et le TFL. 


\subsubsection{Coupe du col fémoral}

La coupe du col fémoral était réalisée comme l'a décrit Röttinger $(15,16)$ : à l'aide d'une double coupe du col en place avant toute manœuvre de luxation articulaire, permettant de faciliter l'exposition acétabulaire (Figure 12). La validation du niveau de coupe du col utilisait, comme référence, la palpation du petit trochanter en luxant transitoirement le membre en avant (position de «quatre »), également décrit par Hansen et al en 2011 (62) (Figure 11)

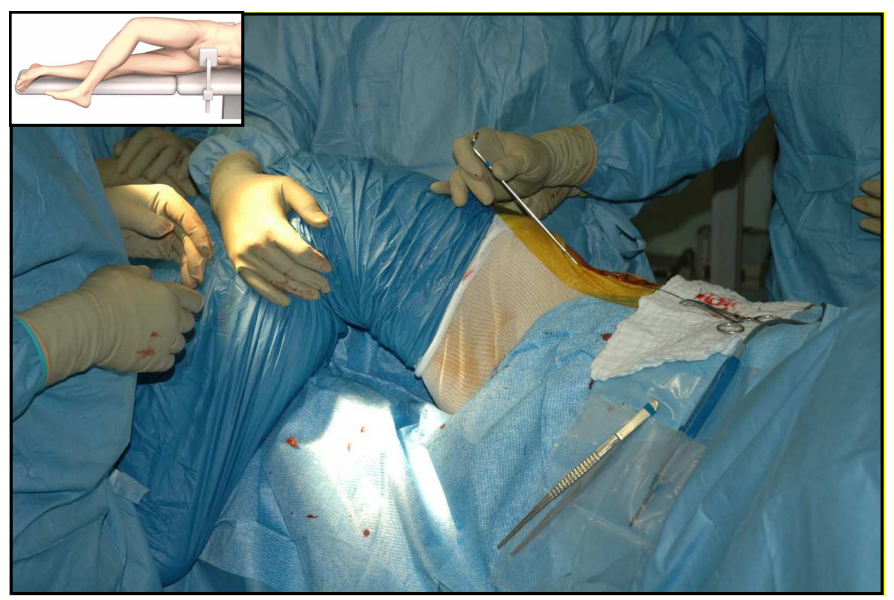

Figure 11: luxation antérieure

\subsubsection{Temps acétabulaire}

Le temps acétabulaire ne présentait pas de spécificité. L'exposition était satisfaisante en dehors de difficultés pouvant être rencontrées dans le cas des hanches protrusives ou des coxa vara (Figure 12).
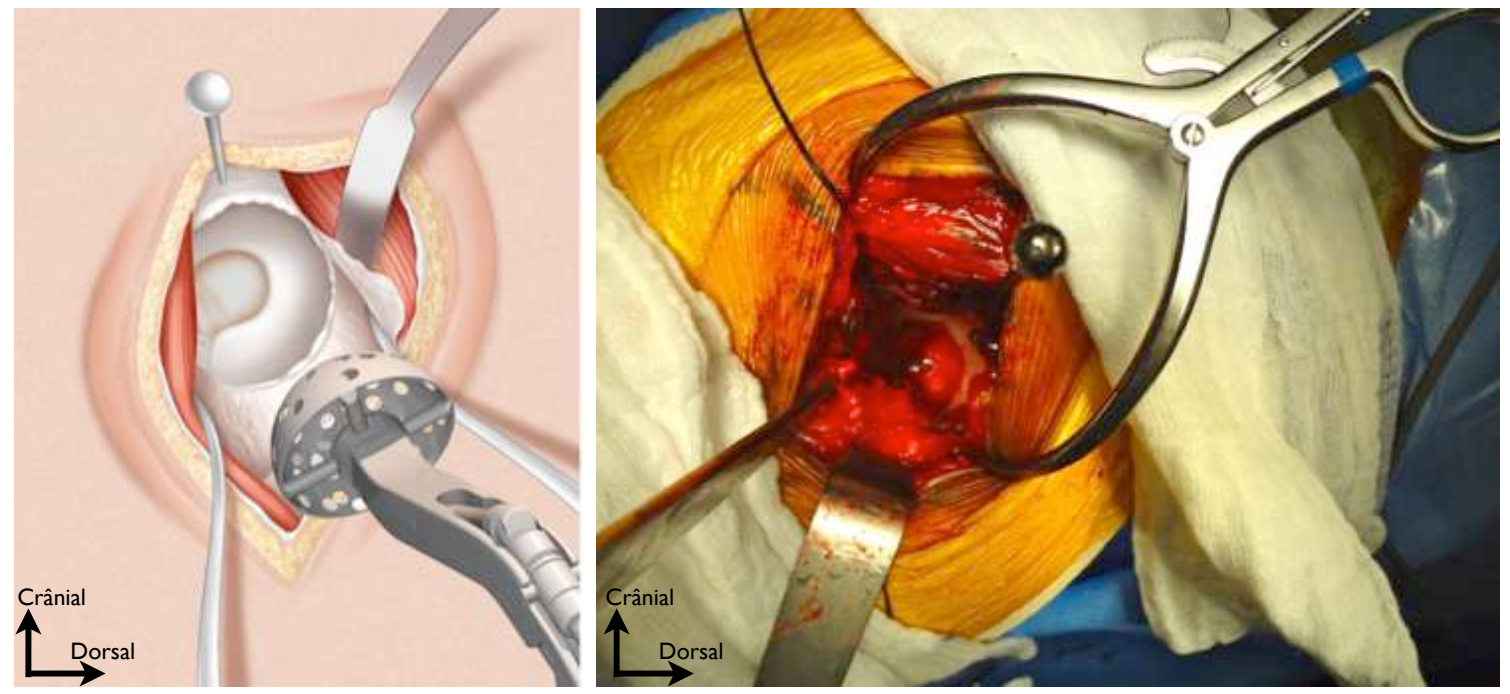

Figure 12: Temps acétabulaire 


\subsubsection{Temps fémoral}

L'exposition et le temps fémoral étaient plus difficiles. L’installation était modifiée (Figure 13) afin de positionner le membre inférieur, luxé en arrière, en adduction associé à une légère rotation externe, sans extension. L'exposition de la tranche de section fémorale était permise par 2 écarteurs contre coudés : un élévateur en appui au niveau de l'éperon médial sous cervical, et un Hohman glissé sur le lambeau capsulaire postéro supérieur en appui sur la paroi postérieure acétabulaire sous le MF (Figure 13). La suite du temps fémoral était standard mais il veillait à éviter tout risque de complication à type de fausse route métaphysaire fémorale proximale (Figure13).

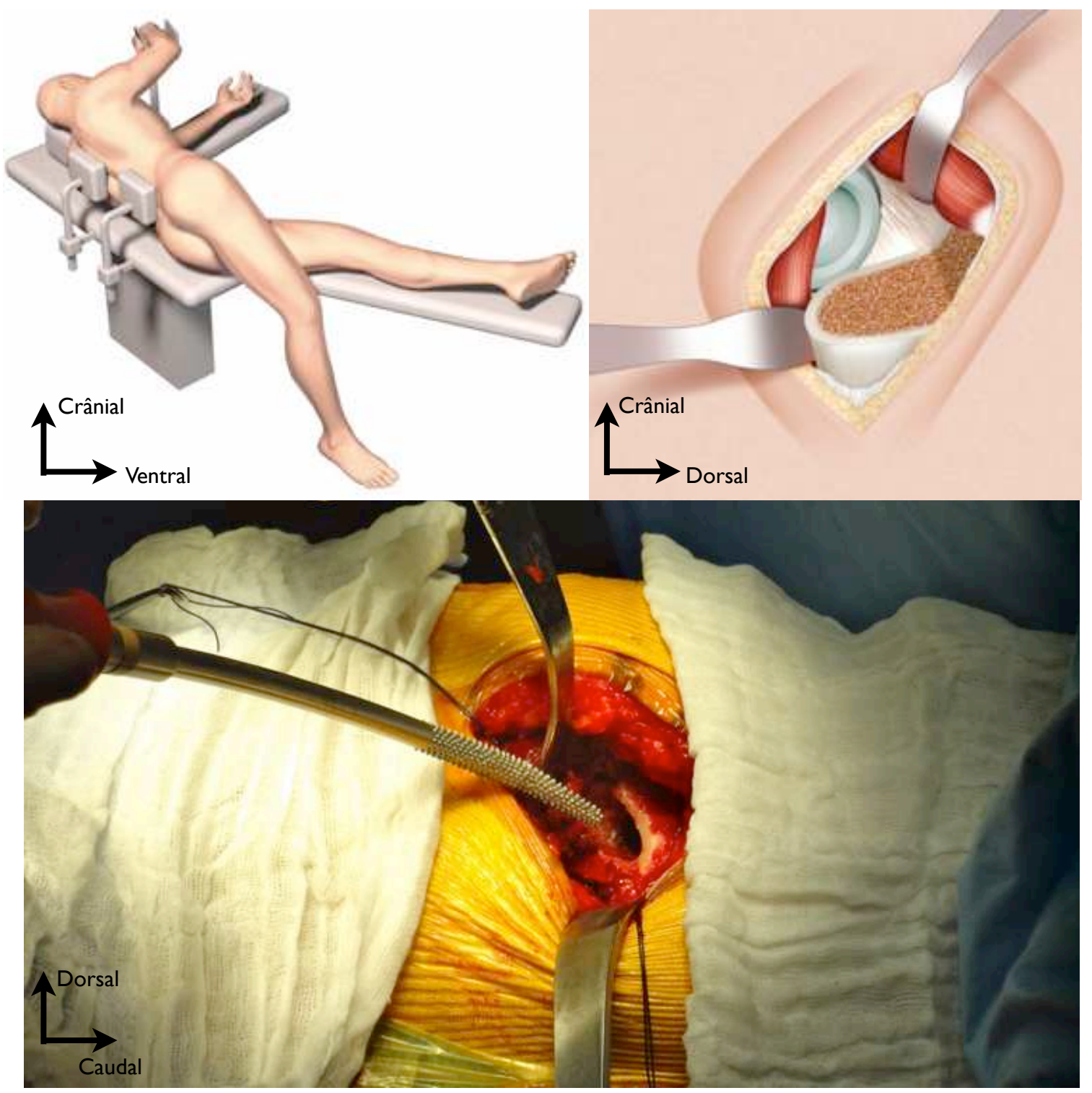

Figure 13: Temps fémoral : installation et exposition. 


\subsubsection{Fermeture et protocole post opératoire}

Les implants mis en place, la fermeture sur un drain de redon ne nécéssitait pas de suture musculaire. Seule l'aponévrose du fascia lata était suturée, puis l'incision cutanée en deux plans séparés.

Les soins post opératoires obéissaient au protocole du service: ablation du redon et reprise de l'appui protégé à 48 heures. Le sevrage progressif et rapide des béquilles était obtenu dans les quinze premiers jours, compte tenu de l'absence de section musculaire. Une anticoagulation préventive était prescrite pendant 5 semaines. Les patients avaient le choix du retour à domicile ou d'un séjour en centre de convalescence. Aucune séance de rééducation n'était prescrite avant la consultation de la $6^{\text {éme }}$ semaine post opératoire.

\subsection{Critère de jugement principal}

Le critère de jugement principal était double :

Clinique : il s'agissait des scores PMA $(63,64)$, Harris (65) avec notamment les caractéristiques de l'appui monopodal, de la présence ou non d'une boiterie de Trendelenburg (24) et de la force d'abduction de la hanche.

Para clinique : l'analyse des lésions musculaires du MF et du TFL était réalisée par IRM.

\subsection{Analyse clinique}

Tous les patients étaient évalués lors des consultations du $3^{\text {ème }}$ et $12^{\text {ème }}$ mois post opératoire par un examinateur unique et indépendant du chirurgien. L'évaluation chiffrée des scores de PMA $(63,64)$ et la cotation fonctionnelle de Harris (65) étaient réalisées (cf annexes).

L'analyse clinique du bilan musculaire des abducteurs de hanches était recueillie: les caractéristiques de l'appui monopodal (signe de Trendelenburg; stable et symétrique, asymétrique, instable), la présence d'une boiterie de Trendelenburg et la force musculaire d'abduction de hanche étaient évaluées.

Le testing musculaire de la force d'abduction volontaire maximale de la hanche, était réalisé en décubitus latéral droit et gauche. Un dynamomètre, à traction mécanique, PESOLA ${ }^{\circledR}$ 
Macro-Line 80490 (PESOLA AGC/ Baar/ Suisse) (Figure 14) était accroché à la cheville du patient. L'abduction isométrique active maximale contre résistance, en maintenant le dynamomètre immobile, était réalisée 3 fois à partir de la position horizontale du membre. Ce testing musculaire est de type statique versant concentrique, également appelé «make test ». Les mesures étaient prises par lecture directe de l'examinateur. La moyenne des 3 mesures était retenue pour chaque membre. Le dynamomètre était préalablement étalonné par le fabricant et vendu pour une précision de $\pm 0,3 \%$.

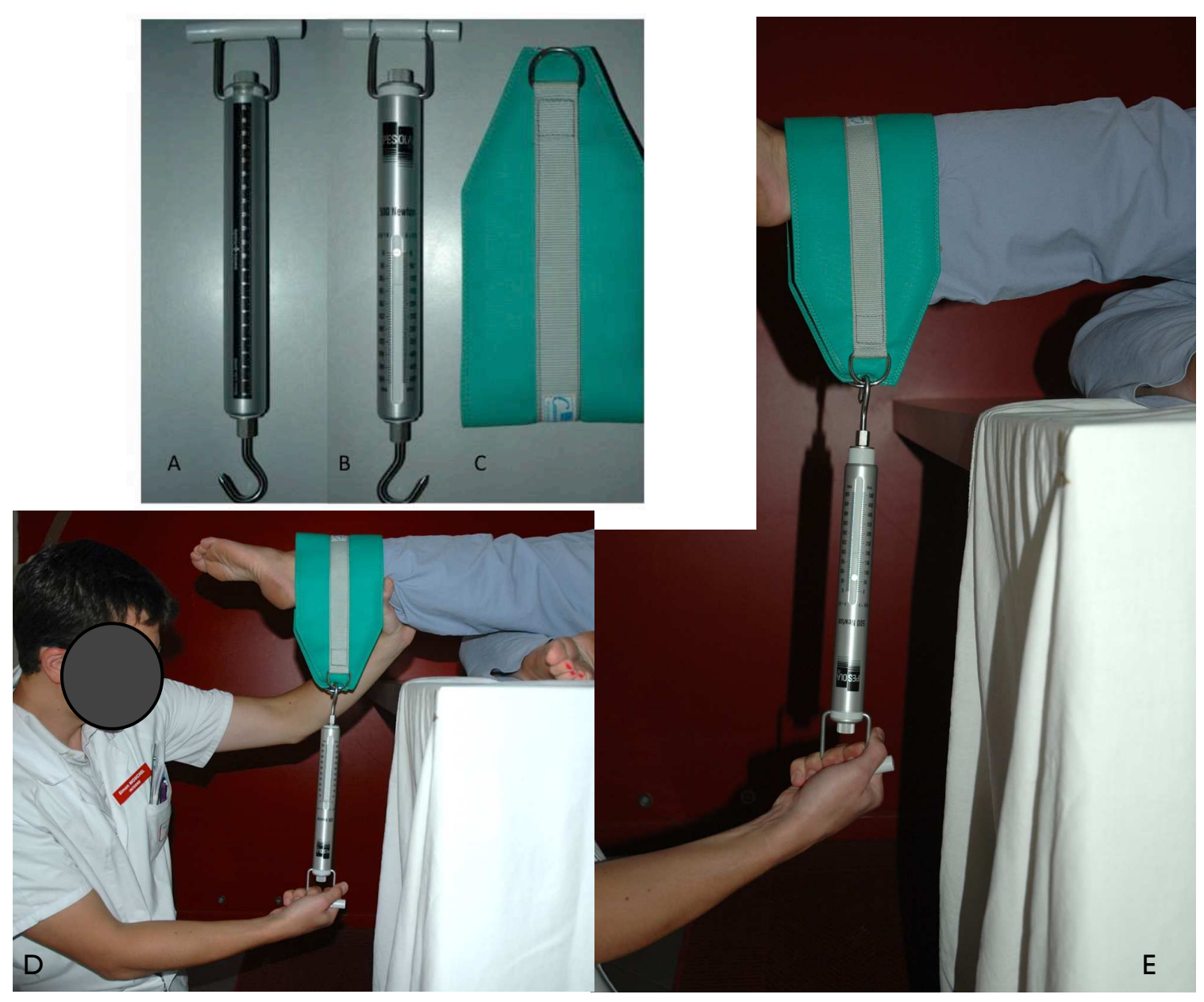

Figure 14: Illustration du dynamomètre utilisé et du testing musculaire.

$\boldsymbol{A}$ : vue postérieure du dynamomètre, avec règle graduée de 0 à $21 \mathrm{~cm} ; \boldsymbol{B}:$ vue antérieure du dynamomètre avec double graduation de la force (à gauche : tous les $10 \mathrm{~N}$; à droite tout les $5 \mathrm{~N})$, pour une force maximale de 500 Newtons ; $\boldsymbol{C}$ : étui utilisé permettant l'accrochage du dynamomètre à la cheville du patient $; \boldsymbol{D}$ : position de repos avant testing ; $\boldsymbol{E}:$ testing de la force musculaire active d'abduction statique versant concentrique. 


\subsection{Analyse radiologique}

Toutes les IRM étaient réalisées par le Dr J.M. Bondeville au sein du cabinet de radiologie GIE IRM Ormeaux Vauban (Le Havre / Seine Maritime/France). La machine utilisée était un model SIGNA ${ }^{\mathrm{TM}}$ HDXT 1,5 Tesla de la société General Electric Healthcare (Milwaukee/Wisconsin/USA).

Les séquences IRM étaient recueillies, puis analysées par un radiologue expérimenté, indépendant de l'opérateur.

\subsubsection{Description du protocole d'imagerie}

Chaque examen obéissait au même protocole:

Les IRM de pelvis étaient réalisées chez des patients en décubitus dorsal sur un plan dur. L'antenne de surface était placée au niveau du pelvis. L'examen commencait par la réalisation première d'une séquence de repérage coronale pondérée en T2 STIR / inversion récupération de signal, moins sensible aux artefacts métalliques. Les paramètres utilisés étaient : le temps de répétition $(\mathrm{TR})=3200 \mathrm{~ms}$, le temps d'écho $(\mathrm{TE})=64 \mathrm{~ms}$, le champ de vue $(\mathrm{FOV})=440 *$ $440 \mathrm{~mm}$ et une matrice d'acquisition $=512 * 512$ pixels.

Deux acquisitions étaient ensuite réalisées : La séquence axiale était placée après la séquence de repérage en prenant comme référence le plan axial du pelvis. Le plan coronal était placé orthogonalement au plan de coupe axial afin d'obtenir une acquisition symétrique.

Ces séquences axiales et coronales étaient pondérées en $\mathrm{T} 1$ et $\mathrm{T} 2$, selon un procédé de décomposition itérative du signal de l'eau et de la graisse appelé IDEAL (66-69) par le groupe General Electric Healthcare («Iterative Decomposition of water and fat with Echo Asymmetry and Least-squares estimation »). La séquence IDEAL est décrite comme étant moins sensible aux artefacts métalliques $(70,71)$.

La séquence qui nous permettait d'obtenir la meilleure définition des muscles et de leurs contours était une séquence en T1 IDEAL FSE $(\mathrm{TR}=540 \mathrm{~ms}$; $\mathrm{TE}=11.7 \mathrm{~ms}$; Matrice d'acquisition $=512 * 512$ pixels). Cette séquence permettait dans l'aire du muscle de bien différencier : 


\section{Le tissu musculaire ou tissu contractile,}

Le tissu non contractile. Celui-ci était représenté par :

- Le tissu graisseux. Il apparaissait sous forme d'infiltrat entre les faisceaux du muscle. Ce tissu graisseux était hyper intense dans cette séquence.

- Le tissu conjonctif (fascias musculaires, éléments vasculo-nerveux). Ce tissu est hypo intense dans cette séquence.

L'oedème musculaire apparaissant en cas de lésions secondaires dues à une dénervation était recherché en séquence FAT T2 Ax IDEAL $(\mathrm{TR}=7000 \mathrm{~ms} ; \mathrm{TE}=76.3 \mathrm{~ms}$; Matrice d'acquisition $=256 * 256$ pixels $)(58,69,72,73)$.

L'épaisseur des coupes était de $8 \mathrm{~mm}$ tous les $11 \mathrm{~mm}$ avec l'existence d'un espacement entre chaque coupe afin de diminuer la diaphonie (interférence d'une image avec une autre). Cet espacement ne doit jamais dépasser 1/3 de l'épaisseur de coupe, en revanche il peut être éliminé au détriment du temps d'acquisition. Ainsi, chaque coupe de $8 \mathrm{~mm}$ d'épaisseur était espacée de $3 \mathrm{~mm}$ afin de couvrir l'ensemble du volume musculaire du pelvis (depuis l'épine iliaque antéro supérieure jusqu'au 1/3 moyen du fémur), en un temps acceptable.

Deux acquisitions ont été réalisées pour un temps d'examen de 15 minutes. Aucune injection de produit de contraste n'était nécessaire.

\subsubsection{Modalités pratiques du recueil des informations}

Les informations étaient recueillies de manière standardisée. Pour chaque muscle: nous étudions, à droite et à gauche (étude comparative du côté «sain » par rapport au côté opéré), 2 paramètres principaux:

- L'aire musculaire (mesure du plus grand diamètre en longueur et en largeur, définissant une surface), afin d'analyser la notion d'amyotrophie ou d'hypertrophie musculaire.

- La dégénérescence graisseuse, classée en 5 stades comme l'ont décrit Goutallier et Bernageau (74,75), pour l'analyse musculaire de la coiffe des rotateurs d'une épaule, en scanner puis transposée et validée à l'IRM dans l'exploration musculaire de la hanche par Engelken et al en 2013 (76) : 
Grade 0: absence de travée graisseuse,

Grade 1: fines travées graisseuses,

Grade 2: travées graisseuses moins importantes que le muscle,

Grade 3: autant de muscle que de graisse,

Grade 4: plus de graisse que de muscle.

L'existence d'un oedème de dénervation du muscle TFL était également notée. Cette entité lésionnelle apparaissait sous la forme d'un hyper signal en séquence FAT T2 Ax IDEAL.

Nous avons réalisé pour chaque IRM la même méthodologie d'analyse $(20,77)$ :

Sélection première de la coupe frontale passant par: la crête iliaque, le grand trochanter et montrant le 1/3 distal du moyen fessier (Figure 15).

A partir de cette coupe frontale nous avons choisi les 2 plans de coupes transversales :

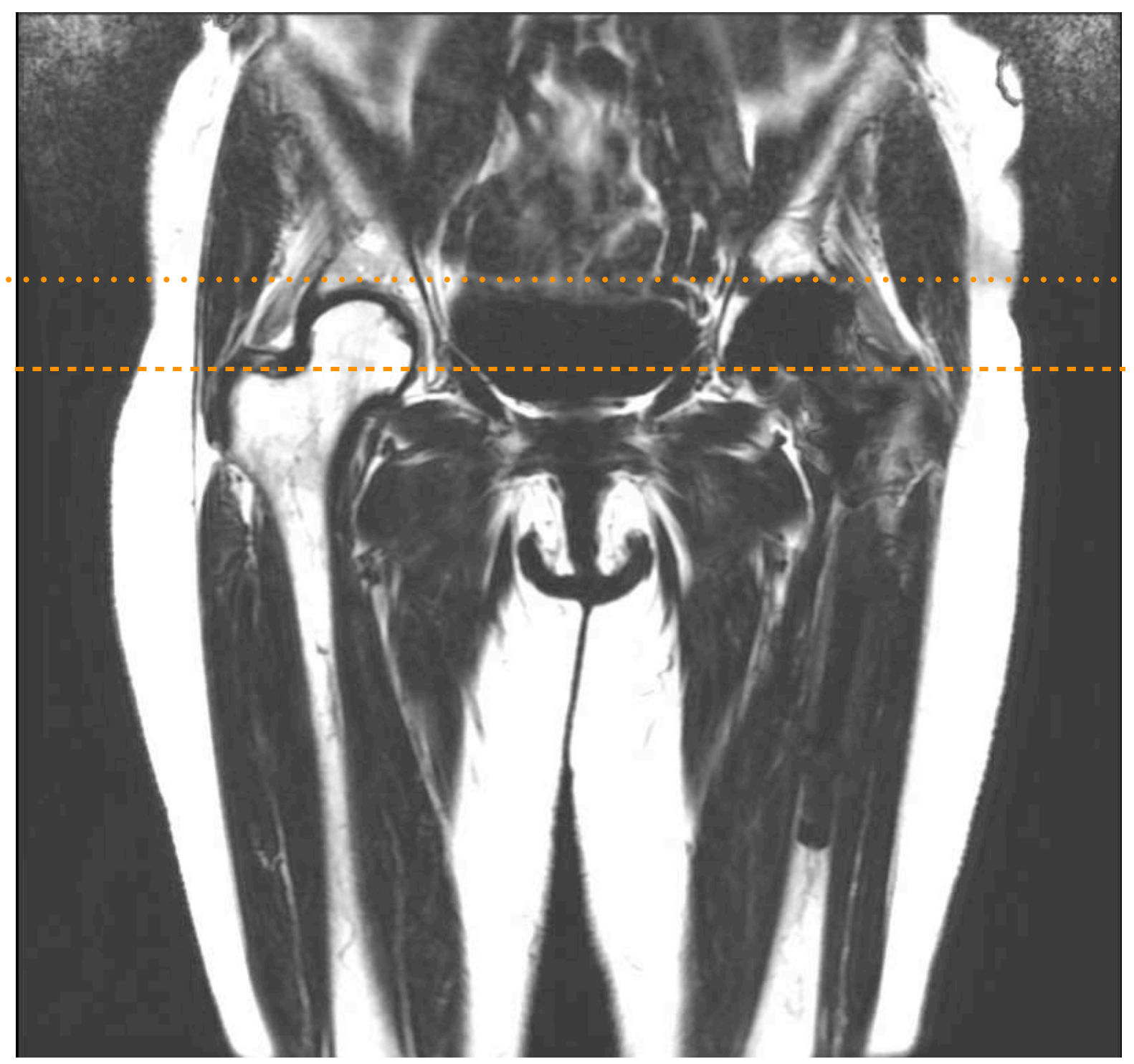

Figure 15: coupe frontale, séquence IRM FAT T1 IDEAL FSE 
L'analyse du moyen fessier était réalisée sur la coupe transversale (Figure 16) passant par le toit du cotyle et le 1/3 distal du MF. Pour le paramètre "Dégénérescence graisseuse", le MF était divisé en 3 tiers : antérieur, moyen et postérieur.

L'analyse du tenseur du fascia lata était réalisée sur la coupe transversale passant par le grand trochanter et la tête fémorale (native ou prothétique) (Figure 17).

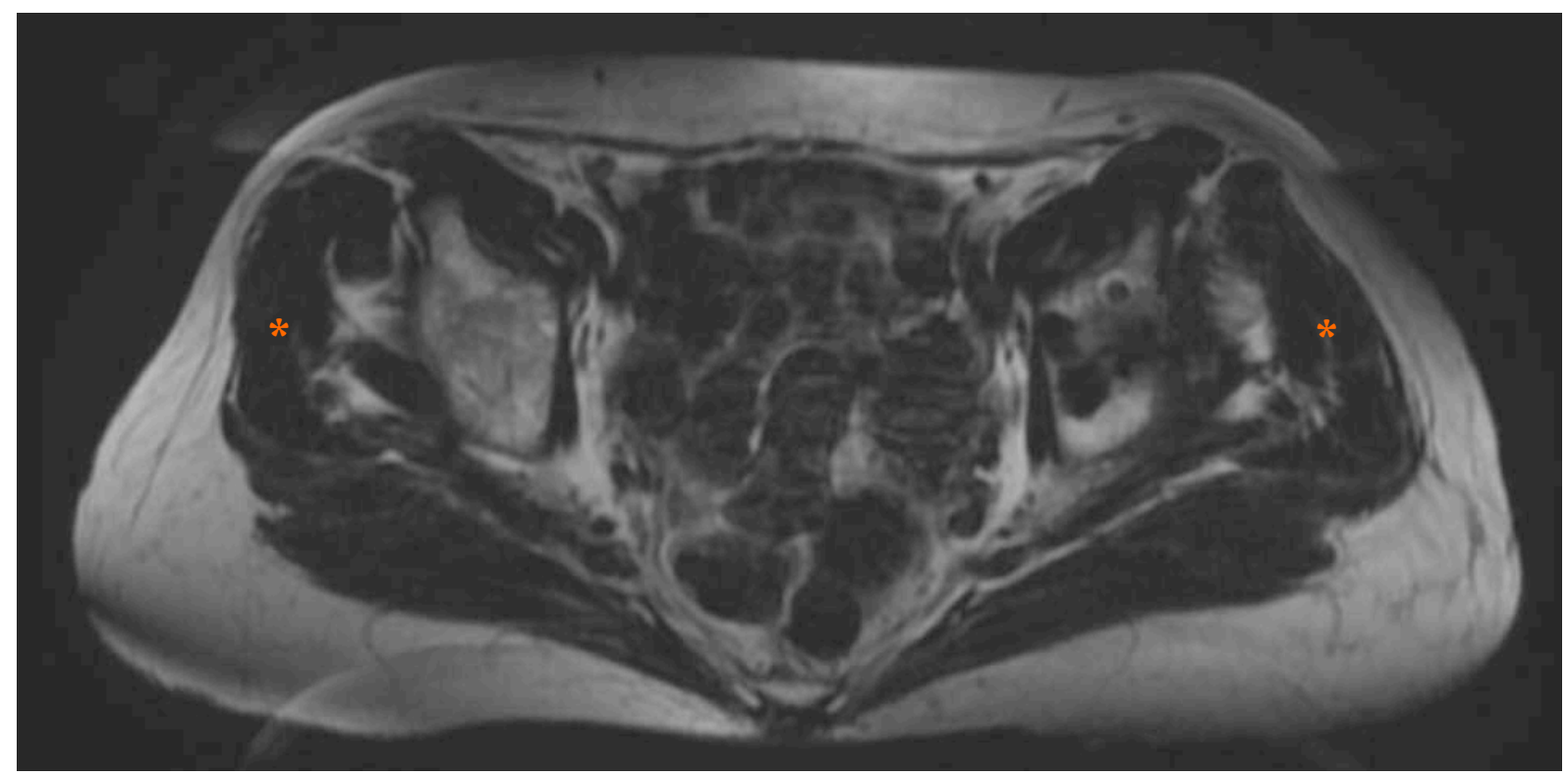

Figure 16: Coupe transversale, séquence IRM FAT T1 IDEAL FSE

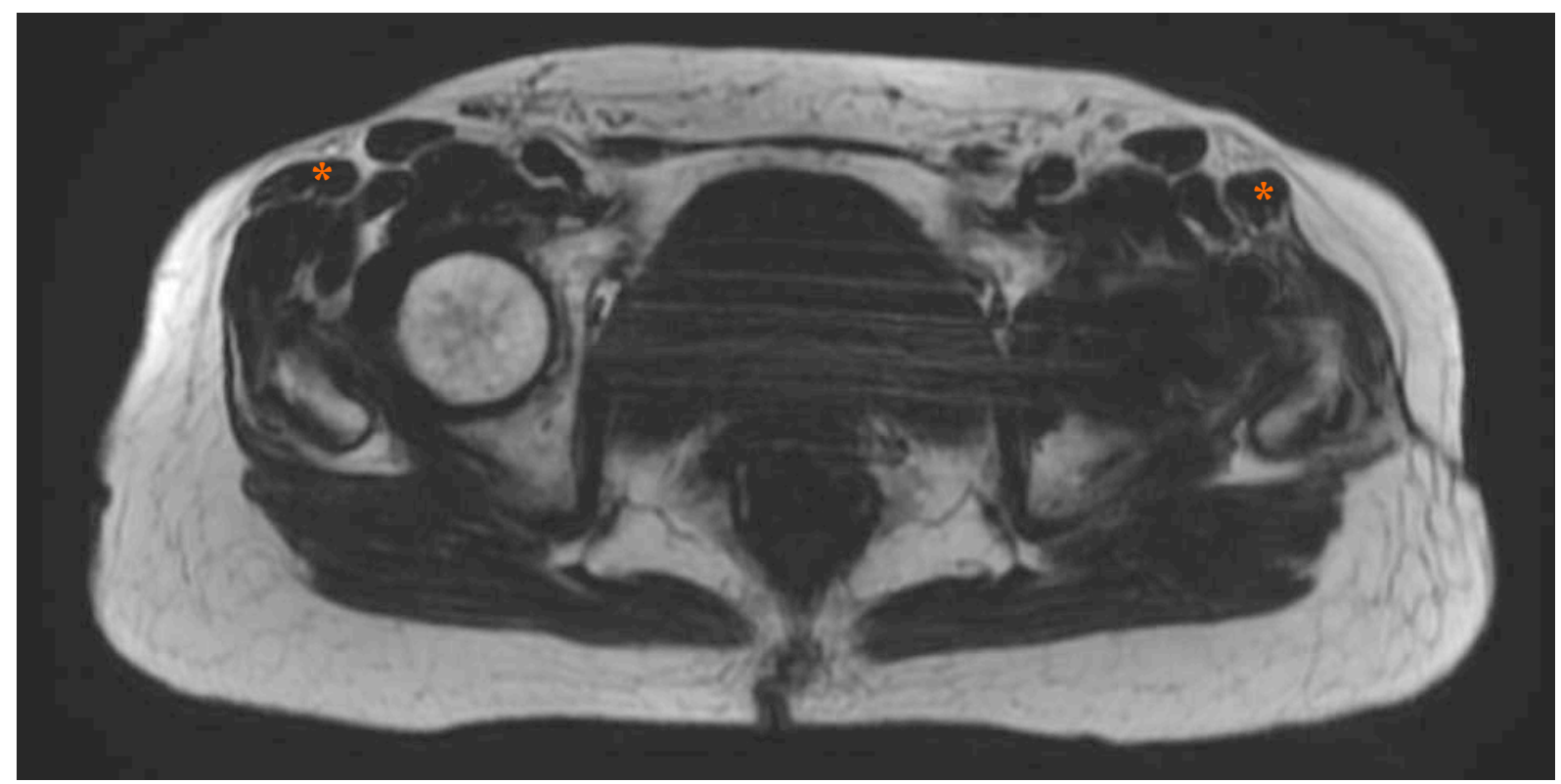

Figure 17: Coupe transversale, séquence IRM FAT T1 IDEAL FSE 


\subsection{ANALYSE STATISTIQUE}

Le recueil des données était effectué sur une feuille de calcul Excel 2010 ${ }^{\mathrm{TM}}$.

L'analyse statistique des données était menée à l'aide du site internet gratuit de tests statistiques en ligne, BiostaTGV http://marne.u707.jussieu.fr/biostatgv/ et du logiciel Microsoft Excel 2010 .

Les tests utilisés étaient fonctions du type de variable et de ses effectifs :

- La recherche d'une différence entre les séries de données quantitatives (aire musculaire et mesure de la force) était réalisée avec le test t de Student pour variables indépendantes, les paramètres étant étudiés sur des sujets différents.

- Les variables quantitatives dont la distribution ne respectait pas la loi normale (score de Harris et PMA) étaient comparées par le test non paramétrique de Wilcoxon.

- La comparaison des variables qualitatives (degré de dégénérescence graisseuse musculaire, boiterie et appui monopodal) était effectuée à l'aide du test exact de Fisher, en raison des faibles effectifs.

La significativité statistique était retenue pour $\mathrm{p}<0,05$.

La description du protocole de l'étude est représentée sur la Figure 18. 


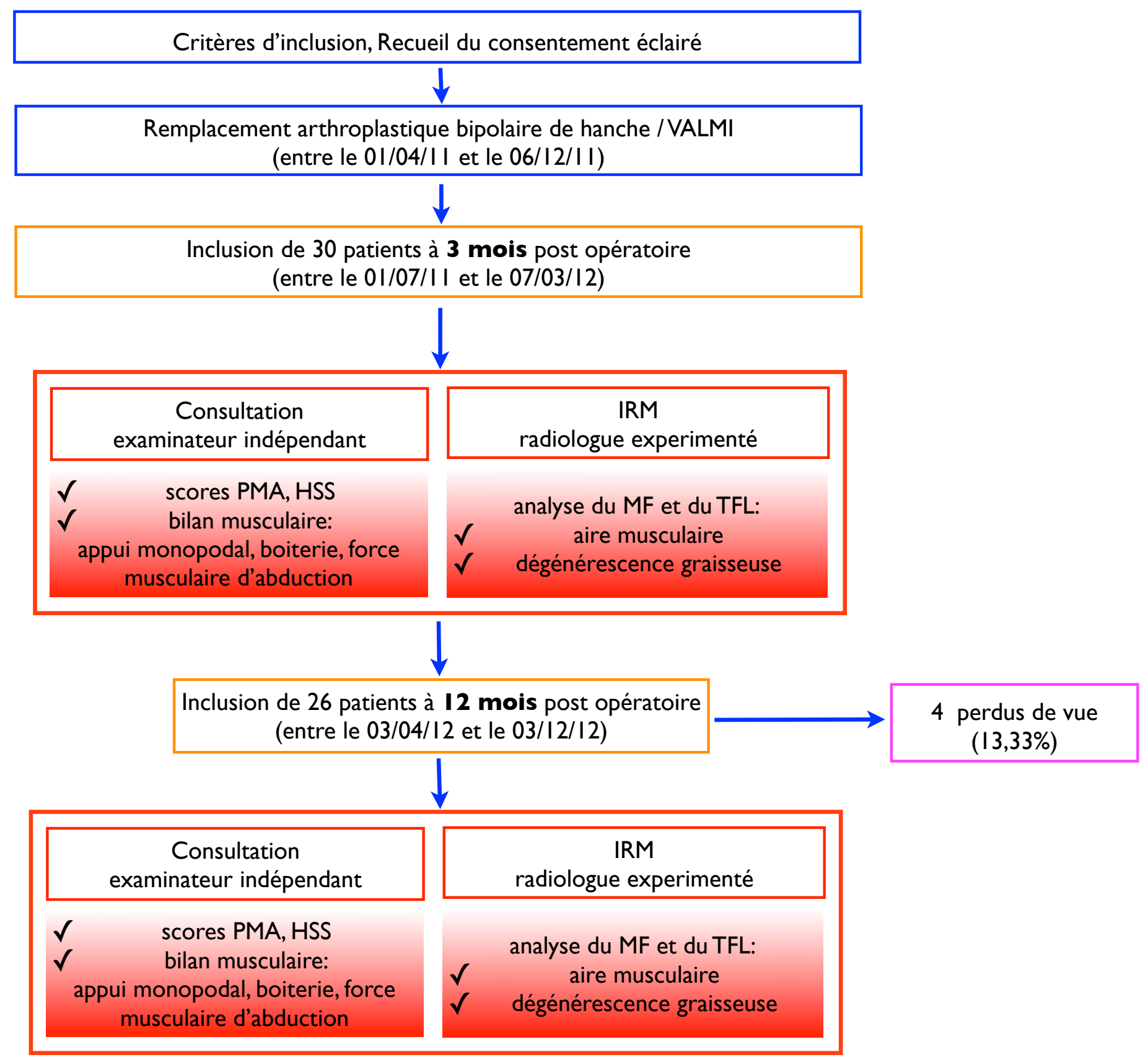

Figure 18: Schématisation du protocole 


\section{RESULTATS}

Vingt six patients opérés par voie antéro latérale mini invasive et 52 IRM ont été revus et analysés à travers cette étude prospective continue au $12^{\text {éme }}$ mois post opératoire.

\subsection{Clinique}

\subsubsection{Scores PMA \& Harris}

La récupération algo-fonctionnelle évaluée par le score PMA, à 3 et 12 mois, était similaire. Il n'était retrouvé de différence significative entre ces 2 bornes temporelles pour ce score.

L'évaluation clinique par le score de Harris était significativement meilleure à 12 mois post opératoire qu'à 3 mois (p=0.047) (Tableau 2).

Tableau 2: résultats scores PMA et Harris

\begin{tabular}{|l|c|c|r|}
\hline $\begin{array}{l}\text { Effectif total : } \mathrm{n}=26 \\
\text { patients }\end{array}$ & $\begin{array}{c}\text { 3 mois } \\
\text { médiane }(\text { mini-maxi) }\end{array}$ & $\begin{array}{r}\text { 12 mois } \\
\text { médiane }(\text { mini-maxi) }\end{array}$ & $\begin{array}{r}\text { Valeur } \mathrm{p} \\
\text { Test de Wilcoxon }\end{array}$ \\
\hline Score PMA & $17(11-18)$ & $18(11-18)$ & 0.121 \\
\hline Score de Harris & $93.5(61-100)$ & $98(65-100)$ & $\mathbf{0 . 0 4 7}$ \\
\hline
\end{tabular}

\subsubsection{Appui monopodal, Trendelenburg et Force musculaire d'abduction}

L'évaluation clinique du bilan musculaire des abducteurs de hanche est recueillie dans les tableaux 3 et 4 .

Aucune différence n'existait entre 3 et 12 mois, pour les paramètres qualitatifs du caractère de 1'appui mono podal et de la boiterie (Tableau 3). La récupération fonctionnelle efficace des muscles stabilisateurs dynamiques du pelvis était précoce. $92 \%$ des patients opérés, présentaient dès le $3^{\text {ème }}$ mois, un appui monopodal stable et symétrique (position statique), et $96 \%$ des patients n'avaient pas de bascule du pelvis à la marche. 
Un seul et unique patient présentait un appui monopodal instable associé à une boiterie de Trendelenburg à l'examen du $3^{\text {mée }}$ et $12^{\text {éme }}$ mois. Un autre patient a gardé une asymétrie à l'appui monopodal.

Tableau 3: Résultats appui monopodal (AMP) \& boiterie

\begin{tabular}{|l|l|l|l|r|}
\hline \multicolumn{2}{|l|}{ Effectif total : $\mathrm{n}=26$ patients } & 3 mois & \multicolumn{1}{|c|}{12 mois } & $\begin{array}{r}\text { Valeur } \mathrm{p} \\
\text { Test de Fisher }\end{array}$ \\
\hline $\begin{array}{l}\text { AMP } \\
(\%)\end{array}$ & Stable et symétrique & $24(92)$ & $24(92)$ & $\mathrm{p}=1$ \\
\cline { 2 - 5 } & Asymétrique & $1(4)$ & $1(4)$ & $\mathrm{p}=1$ \\
\cline { 2 - 5 } & Instable & $1(4)$ & $1(4)$ & $\mathrm{p}=1$ \\
\hline $\begin{array}{l}\text { Boiterie de Trendelenburg } \\
(\%)\end{array}$ & $1(4)$ & $1(4)$ & $\mathrm{p}=1$ \\
\hline
\end{tabular}

La mesure dynamométrique de la force $(\mathrm{N})$ d'abduction des 2 hanches était significativement plus importante du côté non opéré au $3^{\text {éme }}$ mois $(\mathrm{p}=0.038)$. Aucune différence significative n'était retrouvée entre les 2 hanches à 12 mois.

L'augmentation de la force d'abduction des 2 hanches entre le $3^{\text {ème }}$ et le $12^{\text {ème }}$ mois post opératoire n'était pas significative (Tableau 4).

Au $12^{\text {ème }}$ mois, le testing musculaire dynamométrique n'a pu être réalisé, que chez un seul patient. La force musculaire d'abduction était alors réalisée selon la cotation musculaire internationale. Elle était évaluée à $3 / 5$ de manière symétrique («mouvement actif contre pesanteur »). Il s'agissait du même patient qui présentait un AMP instable associé à une boiterie (Tableau 3 ).

Tableau 4: résultats des mesures de la force d'abduction $(\mathbf{N})$

\begin{tabular}{|c|c|c|c|}
\hline $\begin{array}{c}\mathrm{n}=26 \text { patients } \\
(* \mathrm{n}=25 \text { patients à } 12 \text { mois })\end{array}$ & $\begin{array}{l}\text { côté PTH } \\
\text { Force }(\mathrm{N})\end{array}$ & $\begin{array}{c}\text { Hanche native } \\
\text { Force }(\mathrm{N})\end{array}$ & $\begin{array}{r}\text { Valeur } \mathrm{p} \\
\text { Test de } t \text { student }\end{array}$ \\
\hline $\begin{array}{c}\mathbf{3} \text { mois } \\
\text { moyenne } \pm \text { écart type }\end{array}$ & $66,46 \pm 21,5$ & $82,92 \pm 30.9$ & $p=0.038$ \\
\hline $\begin{array}{c}12 \text { mois* } \\
\text { moyenne } \pm \text { écart type }\end{array}$ & $75.43 \pm 27.8$ & $89.57 \pm 31.9$ & $\mathrm{p}=0.116$ \\
\hline $\begin{array}{c}\Delta \text { (3 mois - } 12 \text { mois) } \\
\text { Valeur p Test de t student }\end{array}$ & $\mathrm{p}=0.224$ & $\mathrm{p}=0.472$ & \\
\hline
\end{tabular}




\subsubsection{Aspect cicatriciel}

Parmi les 26 patients opérés et retenus dans cette étude, l'aspect de la cicatrice à 3 et 12 mois post opératoire était noté « sans particularité » pour 21 patients.

Cinq patients ( 1 homme, 4 femmes) ont par ailleurs, présenté une zone de «dépression » clinique au 1/3 moyen de la cicatrice. Aucun syndrome inflammatoire local, ni aucune douleur particulière n'y était associée. Outre l'aspect esthétique, les patients concernés ne semblaient guère y porter attention. Aucune relation statistique n'a pu être mise en évidence entre cet aspect cicatriciel et les autres paramètres cliniques et IRM étudiés dans ce travail. A la relecture de l'imagerie, il semble que seule une atrophie de la graisse sous cutanée soit retrouvée. Le diagnostic retenu était une «lipodystrophie» ou «lipoatrophie » cicatricielle sur le trajet de la voie d'abord chirurgicale (Figure 19).
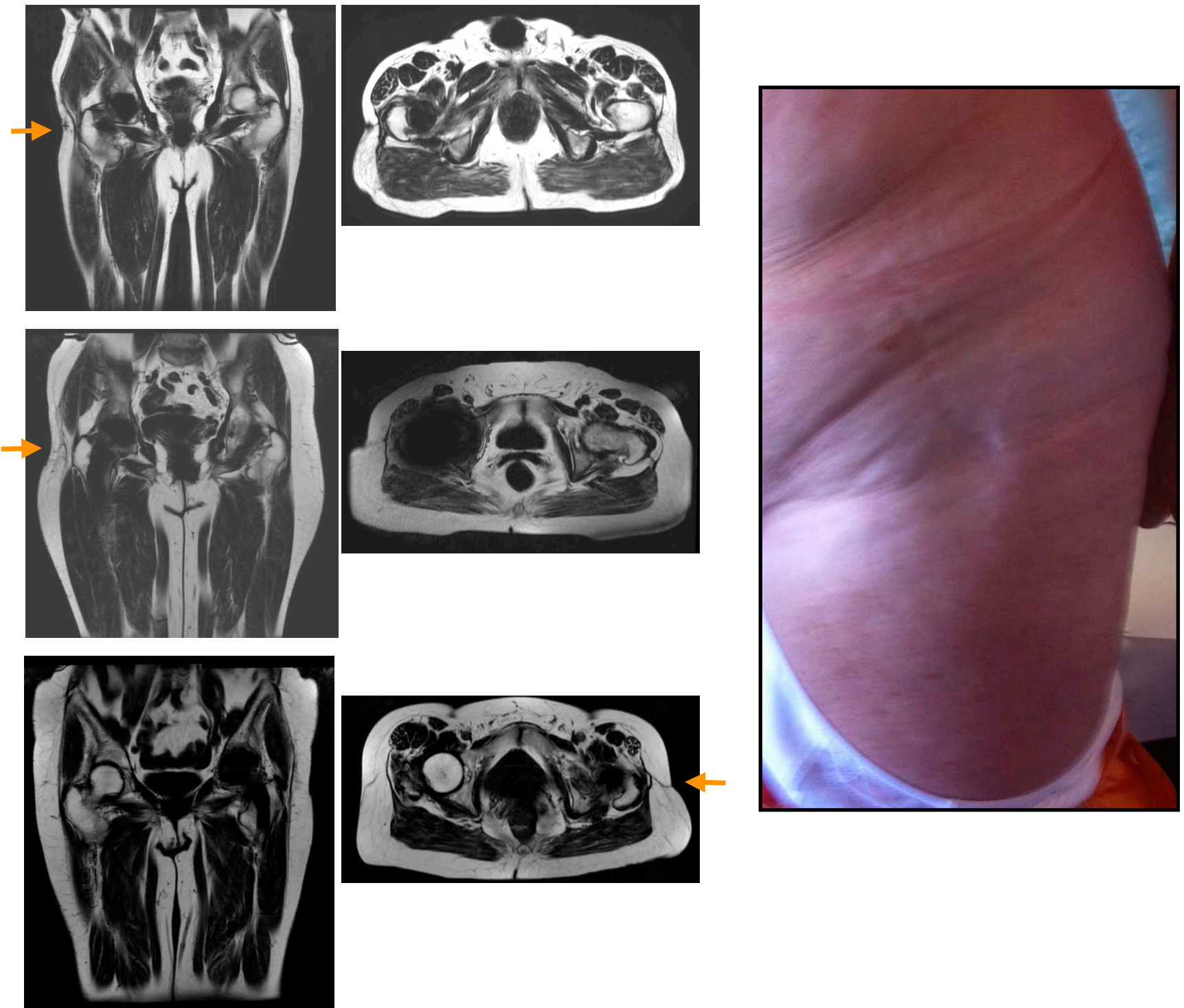

Figure 19: lipoatrophie cicatricielle 


\subsection{IRM}

\subsubsection{Trophicité musculaire}

La trophicité du muscle moyen fessier et tenseur du facia lata (TFL) était étudiée par la mesure de l'aire musculaire $\left(\mathrm{cm}^{2}\right)$ sur la coupe IRM transversale correspondante (cf. Chapitre 3.9.2).

Sur les 52 IRM analysés, nous n'avons retrouvé pour le muscle moyen fessier (Tableau 5) et pour le TFL (Tableau 6):

- Aucune différence significative de la trophicité musculaire entre le côté opéré et le côté non opéré, que ce soit à 3 mois ou à 12 mois post opératoire.

- Aucune variation significative de la trophicité musculaire au sein d'un même côté entre le $3^{\text {eme }}$ et le $12^{\text {eme }}$ mois post opératoire. 
Tableau 5: Aires musculaires du moyen fessier à 3 et 12 mois post opératoire

\begin{tabular}{|c|r|r|r|}
\hline $\mathrm{n}=52$ IRM & $\begin{array}{c}\text { côté PTH } \\
\text { aire }\left(\mathrm{cm}^{2}\right)\end{array}$ & $\begin{array}{r}\text { Hanche native } \\
\text { aire }\left(\mathrm{cm}^{2}\right)\end{array}$ & $\begin{array}{r}\text { Valeur } \mathrm{p} \\
\text { Test de } t \text { student }\end{array}$ \\
\hline 3 mois & $26.36 \pm 7.1$ & $28.12 \pm 8$ & $\mathrm{p}=0.427$ \\
moyenne \pm écart type & $\mathbf{p}$ mois & $26.37 \pm 7.1$ & $28.34 \pm 5.1$ \\
\hline $\begin{array}{c}\text { moyenne } \pm \text { écart type } \\
\text { Valeur } \mathrm{p} \text { Test de t student }\end{array}$ & $\mathrm{p}=0.1$ & $\mathrm{p}=0.911$ & \\
\hline
\end{tabular}

Tableau 6: Aires musculaires du TFL à 3 et 12 mois post opératoire

\begin{tabular}{|c|r|r|r|}
\hline $\mathrm{n}=52$ IRM & $\begin{array}{c}\text { côté PTH } \\
\text { aire }\left(\mathrm{cm}^{2}\right)\end{array}$ & $\begin{array}{c}\text { Hanche native } \\
\text { aire }\left(\mathrm{cm}^{2}\right)\end{array}$ & $\begin{array}{r}\text { Valeur } \mathrm{p} \\
\text { Test de t student }\end{array}$ \\
\hline 3 mois & $6.02 \pm 2.4$ & $7.12 \pm 2.4$ & $\mathrm{p}=0.119$ \\
\hline moyenne \pm écart type & $\mathbf{1 2}$ mois & $6.38 \pm 2.4$ & $6.76 \pm 2.2$ \\
\hline $\begin{array}{c}\text { moyenne } \pm \text { écart type } \\
\text { Valeur } \mathrm{p} \text { Test de t student }\end{array}$ & $\mathrm{p}=0.616$ & $\mathrm{p}=0.587$ & \\
\hline
\end{tabular}




\subsubsection{Dégénérescence graisseuse}

\subsubsection{Moyen fessier}

Les variations du degré de dégénérescence graisseuse du muscle moyen fessier sont représentées dans les tableaux :

- $\quad \mathrm{N}^{\circ} 7,8,9$ pour le $3^{\text {éme }}$ mois post opératoire

- $\quad \mathrm{N}^{\circ} 10,11,12$ pour le $12^{\mathrm{éme}}$ mois post op.

\section{A 3 et 12 mois :}

La voie d'abord influençait l'apparition d'une dégénérescence graisseuse de manière limitée au 1/3 antérieur du MF (Tableaux 7 et 10 ; Figures 20 et 21)

Il n'était pas retrouvé de différence significative de la dégénérescence graisseuse du côté opéré par rapport au côté non opéré, dans le 1/3 moyen et le 1/3 postérieur du MF, que ce soit à 3 ou 12 mois post opératoire (Tableau $8,9,11,12$ ).

\section{Au 1/3 antérieur du moyen fessier :}

Il existait significativement moins de grade 0 («absence de travée graisseuse ») du côté opéré que du côté non opéré ( $\mathrm{p}=0.005)$. La voie d'abord influençait donc l'apparition globale des grades [1, 2, 3 et 4$]$ au 1/3 antérieur du MF.

La répartition des grades 1,2,3, 4 de la dégénérescence graisseuse du 1/3 antérieur du MF montre qu'il n'existait peu ou pas de grades $3[M 3: n=0 ; M 12: n=2(8 \%)]$ et de grades 4 [M3 et M12: $n=1(4 \%)]$ mais plus de grade 1 [M3 et M12: $n=11(42 \%)]$ et de grades 2 [M3 et M12 $\mathrm{n}=5(19 \%)]$.

L'influence de la voie d'abord sur l'apparition de la dégénérescence graisseuse du moyen fessier, semble donc limitée à son $1 / 3$ antérieur et de manière peu importante (essentiellement plus de grade 1 et 2 ). 
Tableau 7: Dégénérescence graisseuse du 1/3 antérieur du muscle MF à 3 mois post opératoire

\begin{tabular}{|l|r|r|r|r|r|r|}
\hline Nombre de patients & Grade 0 & Grade 1 & Grade 2 & Grade 3 & Grade 4 & total \\
\hline $\begin{array}{l}\text { Côté PTH } \\
\text { (\%) }\end{array}$ & $9(35)$ & $11(42)$ & $5(19)$ & 0 & $1(4)$ & $\mathrm{n}=26$ \\
patients \\
\hline $\begin{array}{l}\text { Côté hanche native } \\
\text { (\%) }\end{array}$ & $20(77)$ & $5(19)$ & $1(4)$ & 0 & 0 & $\mathrm{n}=26$ \\
patients \\
\hline Test exact de Fisher & $\mathrm{p}=0.005$ & $\mathrm{p}=0.132$ & $\mathrm{p}=0,191$ & $\mathrm{p}=1$ & $\mathrm{p}=1$ & \\
\hline
\end{tabular}

Tableau 8: Dégénérescence graisseuse du1/3 moyen du muscle MF à 3 mois post opératoire

\begin{tabular}{|l|r|r|r|r|r|r|}
\hline Nombre de patients & Grade 0 & Grade 1 & Grade 2 & Grade 3 & Grade 4 & total \\
\hline $\begin{array}{l}\text { Côté PTH } \\
\text { (\%) }\end{array}$ & $3(12)$ & $15(57)$ & $4(15)$ & $4(15)$ & 0 & $\mathrm{n}=26$ \\
patients \\
\hline $\begin{array}{l}\text { Côté hanche native } \\
\text { (\%) }\end{array}$ & $9(35)$ & $15(58)$ & $2(8)$ & 0 & 0 & $\mathrm{n}=26$ \\
patients
\end{tabular}

Tableau 9: Dégénérescence graisseuse du 1/3 postérieur du muscle MF à 3 mois post opératoire

\begin{tabular}{|l|r|r|r|r|r|r|}
\hline Nombre de patients & Grade 0 & Grade 1 & Grade 2 & Grade 3 & Grade 4 & total \\
\hline $\begin{array}{l}\text { Côté PTH } \\
\text { (\%) }\end{array}$ & $12(46)$ & $8(31)$ & $5(19)$ & $1(4)$ & 0 & $\mathrm{n}=26$ \\
patients \\
\hline $\begin{array}{l}\text { Côté hanche native } \\
\text { (\%) }\end{array}$ & $11(42)$ & $13(50)$ & $2(8)$ & 0 & 0 & $\mathrm{n}=26$ \\
patients
\end{tabular}


Tableau 10: Dégénérescence graisseuse du 1/3 ant du muscle MF à 12 mois post opératoires

\begin{tabular}{|l|r|r|r|r|r|r|}
\hline Nombre de patients & Grade 0 & Grade 1 & Grade 2 & Grade 3 & Grade 4 & total \\
\hline $\begin{array}{l}\text { Côté PTH } \\
\text { (\%) }\end{array}$ & $7(27)$ & $11(42)$ & $5(19)$ & $2(8)$ & $1(4)$ & $\mathrm{n}=26$ \\
patients \\
\hline $\begin{array}{l}\text { Côté hanche native } \\
\text { (\%) }\end{array}$
\end{tabular}

Tableau 11: Dégénérescence graisseuse du 1/3 moyen muscle MF à 12 mois post opératoire

\begin{tabular}{|l|r|r|r|r|r|r|}
\hline Nombre de patients & Grade 0 & Grade 1 & Grade 2 & Grade 3 & Grade 4 & total \\
\hline $\begin{array}{l}\text { Côté PTH } \\
\text { (\%) }\end{array}$ & $6(23)$ & $14(54)$ & $6(23)$ & 0 & 0 & $\mathrm{n}=26$ \\
patients \\
\hline $\begin{array}{l}\text { Côté hanche native } \\
\text { (\%) }\end{array}$
\end{tabular}

Tableau 12: Dégénérescence graisseuse du 1/3 postérieur du muscle MF à 12 mois post opératoire

\begin{tabular}{|l|r|r|r|r|r|r|}
\hline Nombre de patients & Grade 0 & Grade 1 & Grade 2 & Grade 3 & Grade 4 & total \\
\hline $\begin{array}{l}\text { Côté PTH } \\
\text { (\%) }\end{array}$ & $8(31)$ & $14(54)$ & $3(12)$ & 0 & $1(4)$ & $\mathrm{n}=26$ \\
\hline $\begin{array}{l}\text { Côté hanche native } \\
\text { (\%) }\end{array}$ & $9(35)$ & $13(50)$ & $2(8)$ & $1(4)$ & 0 & $\mathrm{n}=26$ \\
patients \\
\hline Test exact de Fisher
\end{tabular}




\section{Dégénérescence graisseuse du 1/3 antérieur du MF à 3 mois post opératoire}

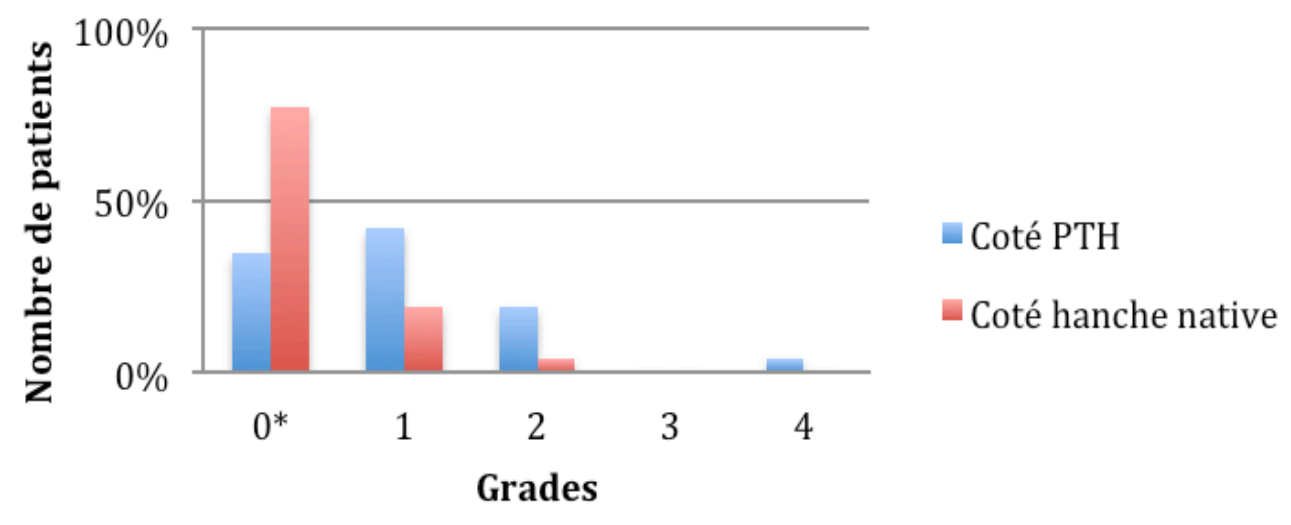

Figure 20: Répartition en pourcentage des grades de la dégénérescence graisseuse du 1/3 antérieur du muscle MF à 3 mois post opératoire

Test exact de Fisher : * $\mathrm{p}=0.005$

\section{Dégénérescence graisseuse du 1/3 antérieur du MF à 12 mois post opératoire}

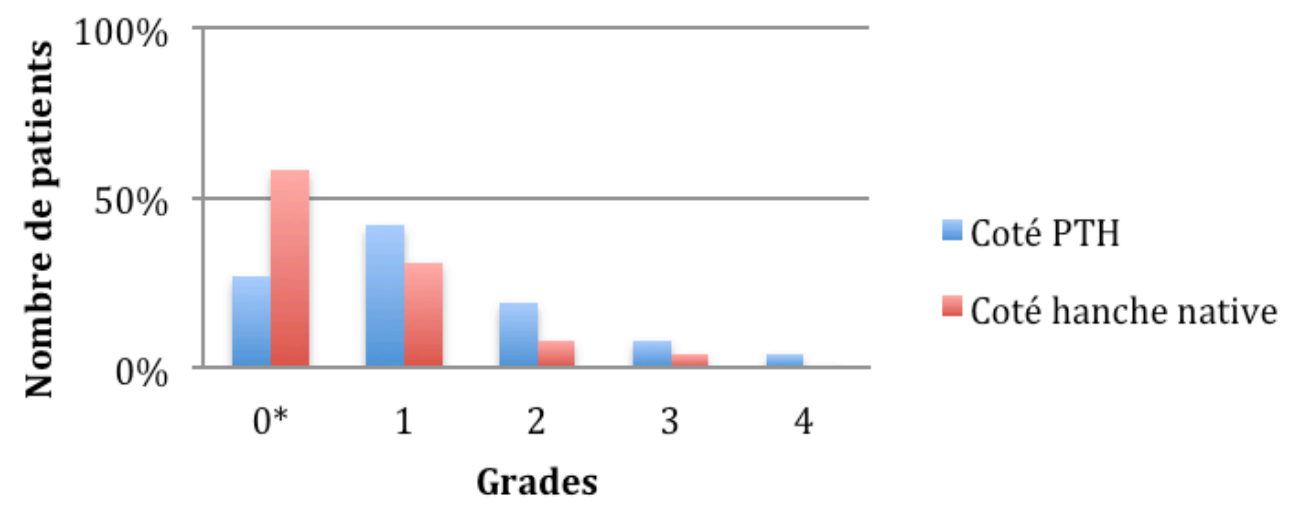

Figure 21: Répartition en pourcentage des grades de la dégénérescence graisseuse du 1/3 antérieur du muscle MF à 12 mois post opératoire

Test exact de Fisher : * p $=0.048$ 


\subsubsection{TFL}

\section{A 3 mois (Tableau 13 et Figure 22) :}

La voie d'abord influençait précisément et de manière significative l'apparition des grades 1 et des grades 2. Le TFL du côté opéré présentait significativement plus de grade $2[\mathrm{n}=30$, $(50 \%)]$ («travées graisseuses moins importantes que le muscle », $\mathrm{p}=0.017$ ) et moins de grade $1[\mathrm{n}=6,(23 \%)]$ ( « fines travées graisseuses », $\mathrm{p}=0.011$ ) que le côté non opéré.

\section{A 12 mois (Tableau 14 et Figure 23) :}

Il était seulement retrouvé un nombre moins important de grade $1[\mathrm{n}=7,(27 \%)](\mathrm{p}=0.048)$ dans le TFL du côté opéré.

L'influence de la voie d'abord sur l'apparition de la dégénérescence graisseuse du TFL, semble donc plus ciblée que pour le MF, avec néanmoins l'apparition de lésions peu importantes (essentiellement grade 1 et 2 ).

Tableau 13: Dégénérescence graisseuse du muscle tenseur du fascia lata à 3 mois post opératoire

\begin{tabular}{|l|r|r|r|r|r|r|}
\hline Nombre de patients & Grade 0 & Grade 1 & Grade 2 & Grade 3 & Grade 4 & total \\
\hline $\begin{array}{l}\text { Côté PTH } \\
\text { (\%) }\end{array}$ & $3(12)$ & $6(23)$ & $13(50)$ & $4(15)$ & 0 & $\mathrm{n}=26$ \\
patients \\
\hline $\begin{array}{l}\text { Côté hanche native } \\
\text { (\%) }\end{array}$ & $3(12)$ & $16(62)$ & $4(15)$ & $3(12)$ & 0 & $\mathrm{n}=26$ \\
patients
\end{tabular}

Tableau 14: Dégénérescence graisseuse du muscle tenseur du fascia lata à 12 mois post opératoire

\begin{tabular}{|l|r|r|r|r|r|r|}
\hline Nombre de patients & Grade 0 & Grade 1 & Grade 2 & Grade 3 & Grade 4 & total \\
\hline $\begin{array}{l}\text { Côté PTH } \\
\text { (\%) }\end{array}$ & $1(4)$ & $7(27)$ & $12(47)$ & $3(12)$ & $3(12)$ & $\mathrm{n}=26$ \\
patients \\
(\%)
\end{tabular}




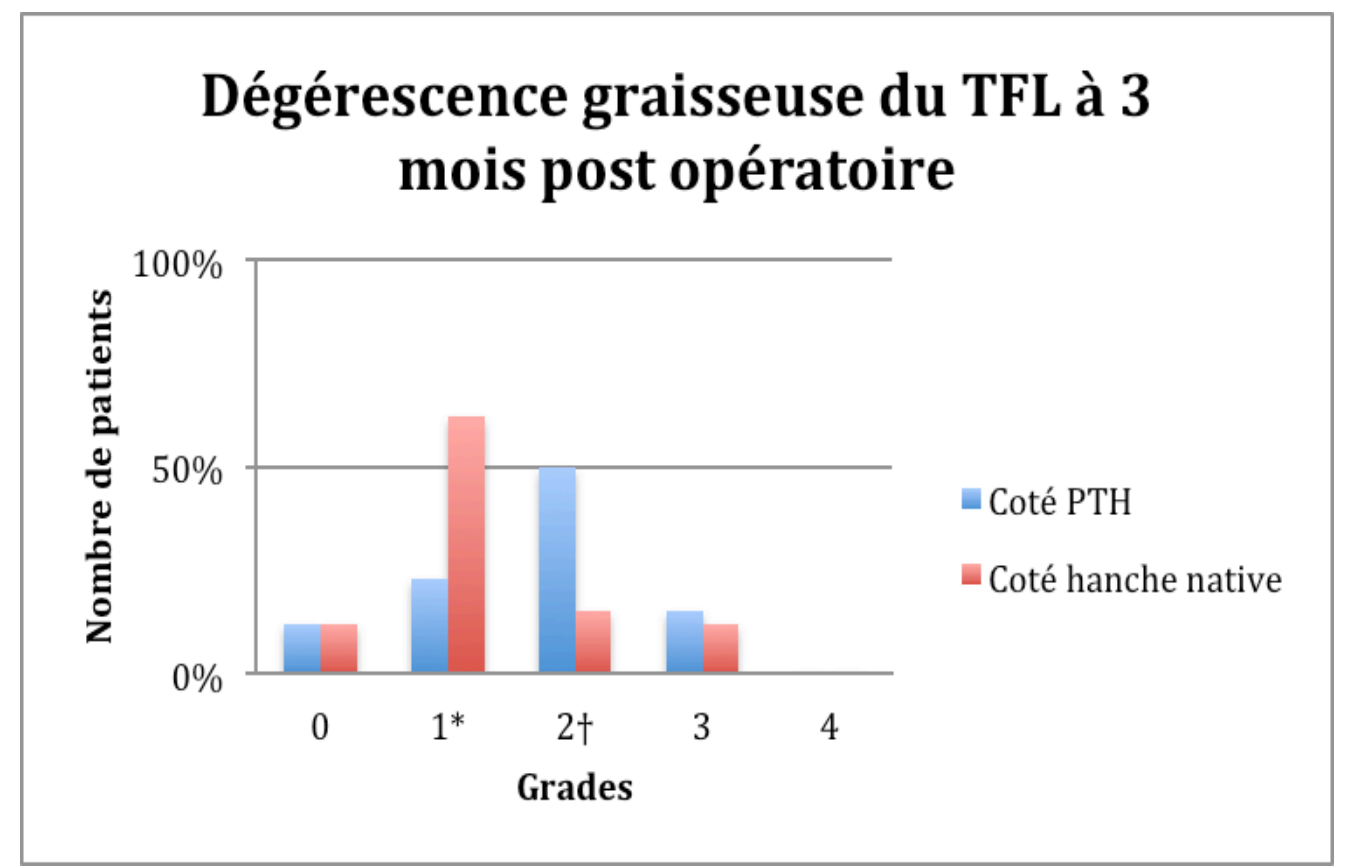

Figure 22: Répartition en pourcentage des grades de la dégénérescence graisseuse du muscle TFL à 3 mois post opératoire

Tests exacts de Fisher: $* \mathrm{p}=0.011 ; \dagger \mathrm{p}=0.017$

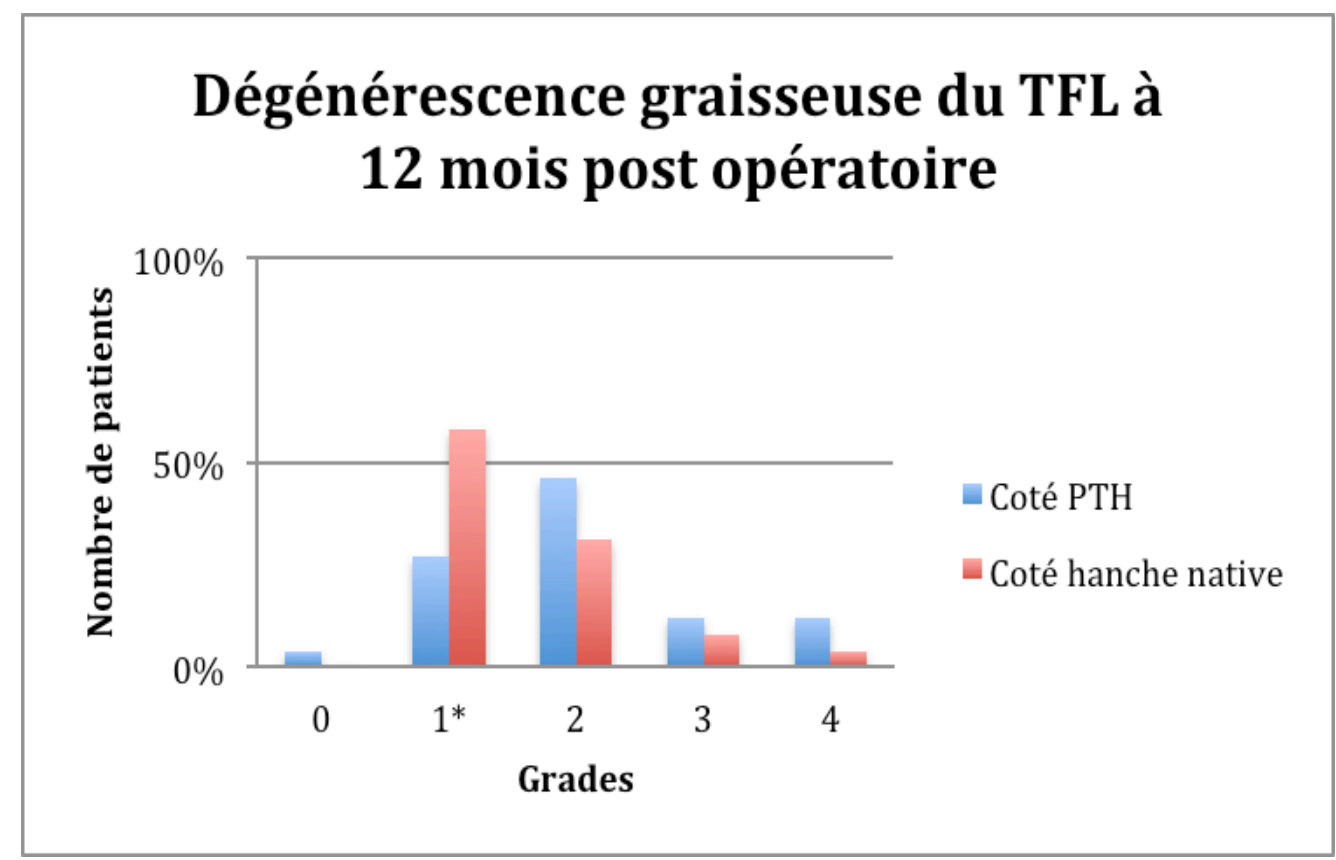

Figure 23: Répartition en pourcentage des grades de la dégénérescence graisseuse du muscle TFL à 12 mois post opératoire

Tests exacts de Fisher : * $\mathrm{p}=0.048$ 
L'analyse de l'oedème musculaire secondaire aux lésions de dénervation nécessitait des séquences plus spécifiques, dites FAT T2 IDEAL. Celles-ci n'ont malheureusement pas toujours été réalisées. Ce type de lésion du TFL était référencé en l'absence de lésions controlatérales. A 3 mois, seul dix sept patients (65\% de notre effectif) ont bénéficié des ces séquences IRM dédiées, contre vingt quatre patients (92\% de notre effectif) au dernier recul de 12 mois. Les résultats des effectifs des patients présentant un aspect d'oedème en hypersignal FAT T2 IDEAL du muscle TFL sont représentés dans le tableau 15.

Nous confirmons la présence de lésions de dénervation du TFL avec un taux comparable entre le $3^{\text {éme }}$ et le $12^{\text {ème }}$ mois post opératoire (p>0.05) (cf. annexe Figure 28).

Tableau 15: effectifs des patients présentant un oedème de dénervation du TFL, du côté opéré

\begin{tabular}{|l|l|l|l|}
\hline n= 26 patients & \multicolumn{1}{|c|}{$\begin{array}{c}\text { Edème de } \\
\text { dénervation du TFL }\end{array}$} & $\begin{array}{c}\text { Absence d'Gdème de } \\
\text { dénervation du TFL }\end{array}$ & \\
\hline $\mathbf{3}$ mois (\%) & $4(24)$ & $13(76)$ & $\mathrm{n}=17$ \\
\hline $\mathbf{1 2}$ mois (\%) & $5(21)$ & $19(79)$ & $\mathrm{n}=24$ \\
\hline
\end{tabular}




\section{DISCUSSION}

Les résultats de cette étude confirment notre hypothèse. L'utilisation d'un abord chirurgical antéro latéral mini invasif entraînait l'apparition de lésions musculaires loco régionales infra cliniques (PMA $\geq 17$; Harris $\geq 90 / 100,92 \%$ d'AMP stable et symétrique, 4\% de boiterie de Trendelenburg, à 3 et 12 mois ; Force musculaire comparable à 12 mois). La voie d'abord entraînait de façon précoce (dès le $3^{\text {ème }}$ mois) des lésions de dégénérescence graisseuse limitées au 1/3 antérieur du moyen fessier (apparition de l'ensemble des grades [1, 2, 3 et 4], p=0.005 à 3 mois et p=0.048 à 12 mois) et de manière peu importante (essentiellement plus de grade 1 et 2). L’influence de la voie d'abord sur l'apparition de la dégénérescence graisseuse du TFL, semblait plus ciblée que pour le MF, avec néanmoins l'apparition de lésions peu importantes (essentiellement grade 1 et 2). L'existence de lésions de dénervation du TFL est également confirmée dans près de $21 \%$ des cas, avec la persistance de celles-ci au $12^{\text {ème }}$ mois. Aucune influence de la voie d'abord sur la trophicité musculaire n'a pu être mise en évidence : aucune hypo ou hypertrophie musculaire compensatrice d'un muscle par rapport à l'autre, n'était retrouvée.

\subsection{Analyse de la méthodologie}

\subsubsection{Clinique}

Les paramètres étudiés étaient choisis pour permettre l'analyse de la récupération fonctionnelle de cette technique chirurgicale ainsi que le respect de la fonction musculaire des principaux abducteurs de la hanche.

Le score de PMA $(63,64)$ constitue une cotation de référence en France, de réalisation simple.

Il évalue 3 critères: la douleur, la mobilité et la stabilité. Elle donne cependant plus d'importance à la mobilité par rapport aux autres critères.

Le score Harris (65) est une classification très utilisée dans la littérature internationale. Elle évalue 5 critères: la douleur, la fonction, les activités, l'absence d'attitude vicieuse et les amplitudes articulaires. Elle est de réalisation plus longue et complexe. Il donne une évaluation prépondérante de la fonction et de la douleur sur la mobilité. 
Ces deux méthodes couramment utilisées dans la littérature, attribuent un score chiffré aux trois principaux paramètres (la douleur, le retentissement fonctionnel et la mobilité de la hanche). Une fois additionnées elles permettent d'obtenir une classification des patients en : « excellent, très bon, bon, moyen, mauvais résultat » afin de pouvoir les comparer à d'autres publications. Cependant, plusieurs études ont analysé une même cohorte de patients à l'aide de plusieurs classifications et scores fonctionnels : Callaghan JJ. et al en 1990 (78), Bryant MJ. et al en 1993 (79) puis Bach CM. et al en 2003 (80). Ces auteurs ont montré que l'interprétation du résultat algo-fonctionnel d'une même cohorte de prothèses de hanche était très variable en fonction des cotations utilisées (Figure 24). Nous pensons qu'il est donc important d'utiliser au moins deux scores différents afin d'apprécier la récupération fonctionnelle des patients.

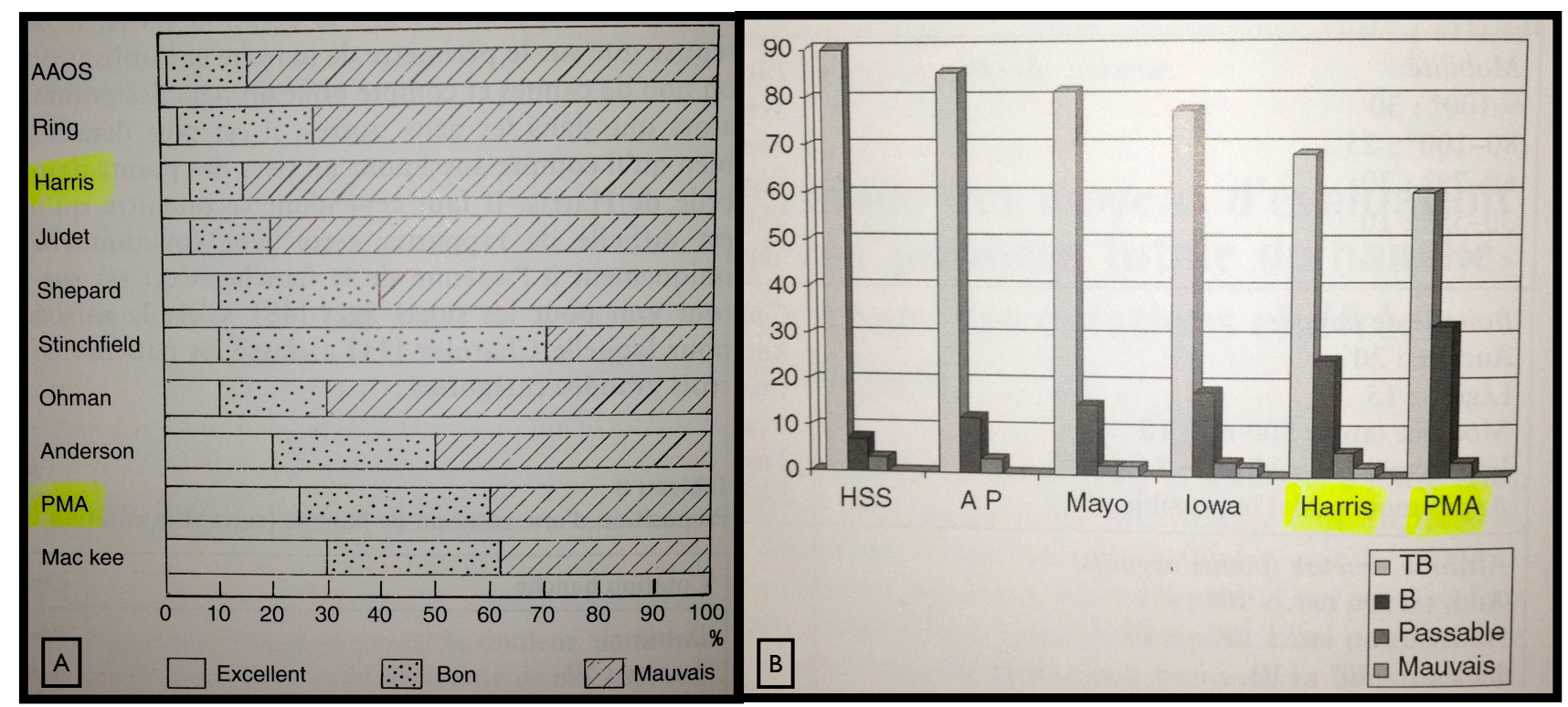

Figure 24: résultats comparés de plusieurs cotations algo-fonctionnelle de hanche (d'après J. Witvoet, (81))

A : Bryant MJ. et al, J Bone Joint Surg 1993 (79); B : Callaghan JJ. et al, J Bone Joint Surg 1990 (78).

Nous avons également choisi de distinguer les principaux paramètres s'intéressant à la stabilité du pelvis. Les caractéristiques de l'appui monopodal (24) (stable et symétrique, asymétrique, instable) ; l'existence ou non d'une boiterie de Trendelenburg et la mesure de la force d'abduction active en décubitus latéral, grâce à un dynamomètre. Ces paramètres ne sont pas spécifiques du muscle moyen fessier et TFL, alors que l'analyse IRM n'a étudié que ces deux muscles (cf. Chapitre 2.1). Nous n'avons pas retrouvé de méthodes cliniques plus fiables et spécifiques, de ces 2 principaux muscles stabilisateurs du pelvis situés sur le trajet de la voie d'abord chirurgicale étudiée. 
L'intérêt du testing dynamométrique est de recueillir une évaluation chiffrée plus précise, lorsque cela était possible, que la cotation musculaire internationale en 5 stades (stade $0=$ paralysie totale $; 1=$ contraction palpable ou visible ; $2=$ mouvement actif, en absence de pesanteur; $3=$ mouvement actif, contre pesanteur ; $4=$ mouvement actif, contre légère résistance; $5=$ mouvement actif, contre résistance complète; NT $=$ non testable). Nous faisons l'analogie avec le testing dynamométrique des muscles de l'épaule, couramment utilisé dans la pathologie musculo tendineuse de la coiffe des rotateurs et intégré dans le Score de Constant et Murley (82)

Par ailleurs, la mesure de la force d'abduction de la hanche peut être évaluée par différents appareils et selon plusieurs positions. Que ce soit dans le cadre d'une surveillance clinique ou de recherche expérimentale, la fiabilité et l'utilisation de manœuvres de testing clinique (83), d'appareils de mesures iso cinétiques $(84,85)$, et de dynamomètres (qu'ils soient manuels ou stabilisés sur un socle) (86-88) est considérée comme bonne et acceptable $(87,88)$. La position du patient durant le testing reste essentielle. Le testing de la force musculaire est plus fréquemment réalisé en décubitus latéral dans le cadre de la pratique clinique (89). Les avantages théoriques du testing en décubitus dorsal seraient la neutralisation de la force de gravité, et la possibilité pour le patient de ne pas avoir besoin de se coucher sur le côté potentiellement douloureux. Par ailleurs, l'orthostatisme serait la position la plus physiologique pour le testing de la force d'abduction de hanche (84). Widler S. et al, en 2009 (90) ont utilisé un prototype de dynamomètre, stabilisé sur un socle en cadre, et comparé ces trois positions (orthostatisme, décubitus dorsal et décubitus latéral). Les conclusions de leur étude étaient que l'évaluation de la force musculaire d'une hanche était plus fiable et reproductible en position de décubitus latéral.

Le principal biais de l'utilisation de ce type d'appareil est qu'il n'est pas automatisé ni informatisé. Il existe un travail réalisé par le service d'évaluation des actes professionnels de l'HAS en novembre 2006 (91) au sujet de « la mesure de la force, du travail et de la puissance musculaire par dynamomètre informatisé et motorisé ». Pour ce type de matériel, il est stipulé que l'étude de reproductibilité est satisfaisante, notamment le moment de force maximale, quand les modalités de standardisation sont respectées (même appareil, même examinateur, lui-même entrainé, avec les mêmes situations). Enfin les groupes articulaires retenus par l'HAS concernent le genou, l'épaule et le rachis. Concernant les autres articulations, le choix de l'examen est laissé à l'appréciation du clinicien. 


\subsubsection{Para clinique}

\subsubsection{Choix de l'imagerie}

Il existe, dans la littérature, différents procédés permettant d'étudier le capital musculaire:

- Mesure de la force musculaire par des dynamomètres et appareils de mesures iso cinétiques $(84,87,91)$

- Mesure du signal à l'électromyographie (17,21,27,92-94)

- Mesure de l'aire musculaire à l'échographie $(95,96)$

- Mesure de l'aire musculaire au scanner (97-101)

- Mesure de l'aire musculaire à l'IRM (20,76,77,92,102-105)

- Mesure de la densité musculaire au scanner $(75,96,106)$

- Mesure de l'intensité du signal musculaire à l'IRM $(76,107,108)$

La Tomodensitométrie est parfois utilisée dans le cadre d'une planification préopératoire d'une PTH ou dans son suivi post opératoire afin de rechercher l'existence de modifications osseuses secondaires à cette arthroplastie $(109,110)$. L'analyse des tissus mous et en particulier de l'appareil musculo tendineux utilise le plus souvent l'IRM: cet examen est non invasif et performant (111-113). Contrairement à l'EMG ou l'échographie, elle permet une analyse objective du capital musculaire, à la fois quantitative et qualitative. Enfin, cet examen ne présente aucune radiation ionisante. 


\subsubsection{Choix du type de séquences et des paramètres}

Afin de diminuer les artefacts métalliques de la prothèse, il est possible d'utiliser plutôt des séquences dites Fast Spin Echo (FSE) que de l'écho de gradient ainsi qu'une pondération plutôt T1 que T2. Comme l'ont décrit Toms et al en 2010 (114) pour une séquence donnée, il est possible d'augmenter la bande passante afin de diminuer le bruit. Pour annuler le signal de la graisse, nous avons préféré les séquences STIR ou IDEAL (66-71) que FS. Enfin, l'utilisation d'une IRM 3 Tesla, qui est plus sensible, risquerait de présenter plus d'artefacts qu'une IRM 1,5 Tesla dans notre cas.

\subsubsection{Paramètres étudiés}

La mesure de l'aire musculaire sur une coupe axiale $(20,76,77,92,102-105)$ et la mesure de l'intensité du signal musculaire $(76,107,108)$ constituent les deux principaux paramètres étudiés dans la littérature pour l'analyse IRM des muscles squelettiques. Ainsi, comme Holmback et al (115) l'ont proposé pour l'étude de la loge antérieure de la jambe et Müller et al $(20,77,103)$ pour l'étude des muscles du pelvis, nous proposons de combiner les informations quantitatives (surface de section musculaire) et qualitative (intensité de signal du muscle) de l'IRM.

\subsubsection{Techniques de mesure de la composante contractile et non contractile du muscle}

Maughan et al $(97,98)$ ont montré que l'aire de section du muscle et son degré de dégénérescence graisseuse visible à l'imagerie TDM mettaient en évidence la présence de lésions musculaires mais également l'existence d'une corrélation avec la fonction musculaire. La densité musculaire est mesurée au scanner en unités Hounsfield (UH). La valeur de la densité du muscle squelettique est connue, elle est comprise entre -30 et 150 UH. Le TDM présente une meilleure résolution spatiale que l'IRM, mais l'IRM est meilleure en terme de résolution de contraste de densité que la TDM. Les informations fournies par la TDM en terme de densité sont donc moindres. L'analyse IRM de la trophicité musculaire est permise dans la littérature par plusieurs procédés : 
- Techniques quantitatives:

- Calcul de l'intensité moyenne du signal dans une région d'intérêt (ROI, Region of interest) (76,103): Une région d'intérêt est placée dans le muscle à étudier sur la coupe transversale de l'IRM. L'intensité moyenne des pixels contenus dans cette région est calculée.

- Calcul de l'intensité moyenne du signal dans le muscle à étudier (103) : L’intensité moyenne des pixels est calculée, non plus dans une ROI, mais dans la totalité de l'aire musculaire, préalablement contourée.

- Méthode de segmentation par intensité de pixel selon Mattei et al (107) : Les valeurs de pixels correspondant au muscle, au fascia et au tissu adipeux sont séparées selon leur intensité, sur un histogramme, selon des seuils prédéfinis afin de déterminer la composante contractile du muscle.

Toutes ces méthodes d'analyses quantitatives de l'intensité du signal IRM présentent un biais majeur : elles sont, en théorie, difficilement reproductibles. En effet, il existe de nombreuses causes de variabilité de l'intensité du signal. Le signal peut être influencé par des facteurs extrinsèques à la machine (qualité de la cage de faraday, environnement électromagnétique, température) et par des paramètres intrinsèques à la machine: les algorithmes de reconstruction différents, les paramètres d'acquisition de l'image que sont le temps de relaxation du proton, le temps d'écho, le nombre d'acquisition ou de séquences. Toute modification d'un ou plusieurs de ces paramètres entraînera une modification du signal. Une possible solution à ce problème pourrait être l'utilisation d'un logiciel tiers visant à normaliser l'intensité du signal par ré-échantillonnage de chaque acquisition. Deux logiciels principaux sont commercialisés et ont fait l'objet de nombreuses publications scientifiques : sliceOmatic 5.0 (TomoVision $\AA$ / Magog / Quebec) et Avizo ${ }^{\circledR}\left(\mathrm{FEI}^{\mathrm{TM}}\right.$ Visualisation Science Group / Mérignac / France). Tous deux utilisent un logiciel de langage scientifique (Matlab / MathWorks ${ }^{\circledR}$ / Naticks / MA) permettant la programmation d'algorithmes et de modèles numériques pour l'interprétation des données. Ces différents logiciels permettent la segmentation numérique fine des images (identification des structures osseuses et des différents tissus mous) pour chacune des acquisitions IRM. Ils permettent également une reconstruction volumique. Cependant, leur utilisation est complexe et la segmentation reste manuelle. Enfin, le processus de reconstruction 3D permettant l'obtention d'une image 
morphologiquement admissible et utilisable, nécessite plusieurs étapes itératives et difficiles (lissage et krigeage). Enfin, Image $\mathrm{J}(116)$ est un autre logiciel de traitement d'image généraliste, gratuit et disponible librement par téléchargement sur internet et produit par le NIH (National Institute of Health). Il ne solutionne cependant pas la nécessité de la normalisation préalable de chaque acquisition IRM qui est elle même, une source théorique de perte d'information. Dans cette étude, nous n'avons pas eu accès à de tels logiciels.

\section{- Techniques qualitatives :}

Comme Müller et al en 2010 (20) ainsi que Engelken F. et al (76) en 2013, nous avons choisi l'utilisation d'une technique visuelle qualitative : la classification de Goutallier et Bernageau 1989 (74) et $1994(75,117)$ en 5 stades, initialement décrite en TDM. Cette classification largement utilisée dans l'étude des muscles de la coiffe des rotateurs de l'épaule est subjective mais suffisante dans la plupart des bilans pré opératoires et des modifications brutales, post opératoires du capital musculaire. De plus, Engelken et al (76) en 2013, ont comparé en utilisant le logiciel Image ${ }^{\circledR}(116)$ cette classification de Goutallier à un nouveau système de classification par «quartile» (grade $0=$ muscle normal ; grade $1=1-25 \%$; grade $2=25$ $50 \%$; grade $3=50-75 \%$; grade $4=75-100 \%$ de graisse dans le muscle). Les auteurs ont étudié et comparé à l'IRM 84 hanches présentant des signes cliniques d'insuffisance du moyen fessier, et 50 autres hanches asymptomatiques. Ils concluaient que ces deux classifications (Goutallier et Quartile) présentaient une grande reproductibilité inter et intra observateur dans l'analyse de l'involution graisseuse des muscles fessiers. Les auteurs valident ainsi l'utilisation de la classification de Goutallier pour l'analyse des muscles fessiers, en IRM et en pratique clinique courante. Nous avons donc préféré cette classification à celle de Lamminen et al (108) décrite en quatre stades (muscles d'aspect normal / lésions dégénératives modérées / lésions dégénératives marquées / lésions dégénératives sévères). 


\subsubsection{Limites de notre étude}

La méthode est dépendante de l'examinateur. Les plans de coupes (frontal et transversal) IRM utilisés étaient choisis par l'examinateur. Le contourage musculaire et la mesure clinique dynamométrique de la force étaient manuels. Malgré l'absence de réalisation d'études de reproductibilité, inter et intra observateur, au sein de ce travail, nos résultats ont été analysés à l'aide des différentes méthodes et études de reproductibilités publiées et validées au sein de la littérature scientifique (cf. Chapitres 5.1.1 et 5.1.2.4).

L'utilisation de logiciels de lecture et de traitement des images, ainsi que d'un dynamomètre numérique automatisé, pourraient permettre d'améliorer la procédure et la rendre moins dépendante de l'opérateur. Cependant, une telle méthodologie s'éloignerait d'autant plus de la pratique clinique courante du chirurgien.

\subsection{Analyse des lésions musculaires induites par la chirurgie.}

\subsubsection{Scores PMA \& Harris.}

L'analyse clinique par les scores PMA et Harris, confirme la récupération fonctionnelle rapide, dés le $3^{\text {ème }}$ mois. Elle était jugée «très bonne » $(\mathrm{PMA}=17)$ ou « excellente » $(\mathrm{PMA}=$ 18, Harris > 90/100). Une amélioration significative du score de Harris est également retrouvée entre 3 et 12 mois.

La figure 25 regroupe et compare les résultats des scores PMA et Harris de ce travail avec les résultats de la littérature récente étudiant ce type de voie d'abord de hanche. Nous indiquons 3 études comparatives randomisées, de fort niveau de preuve (I). Wohlrab et al (118) confirment l'existence d'une amélioration significative précoce $\left(12^{\text {ème }}\right.$ semaines $)$ du score de Harris, des patients opérés avec la voie de Röttinger et par rapport à la voie transglutéale classique. Au $12^{\text {ème }}$ mois, aucune de ces études n'a montré de différence significative avec les autres abords étudiés.

Au dernier recul de 12 mois, la médiane des scores de Harris de notre étude semble meilleure que ceux exposés par ces auteurs $(6,8,56,77,118,119)$. Cette constatation doit, elle aussi, être nuancée par l'absence d'étude statistique sur cet élément de comparaison. 
Au plus long recul, seule une étude de niveau de preuve 1, montre un élément significatif de comparaison avec une autre voie d'abord parmi les différents scores utilisés. White et al (120) ont réalisé en 2009 une étude comparative randomisée entre la voie de Röttinger et la voie mini invasive postérieure. Cette évaluation clinique était menée par la comparaison des résultats des différents scores de Harris, WOMAC et MOS sleep scale, en pré opératoire et pendant 10 consultations post opératoires, sur une période de 2 ans. 600 comparaisons différentes ont été réalisées. Vingt pour cent des comparaisons étaient en faveur de la voie d'abord antéro latérale mini invasive de Röttinger, contre $0.33 \%$ en faveur de la voie d'abord mini postérieure. $79.7 \%$ de ces comparaisons ne retrouvaient aucune différence statistique. 


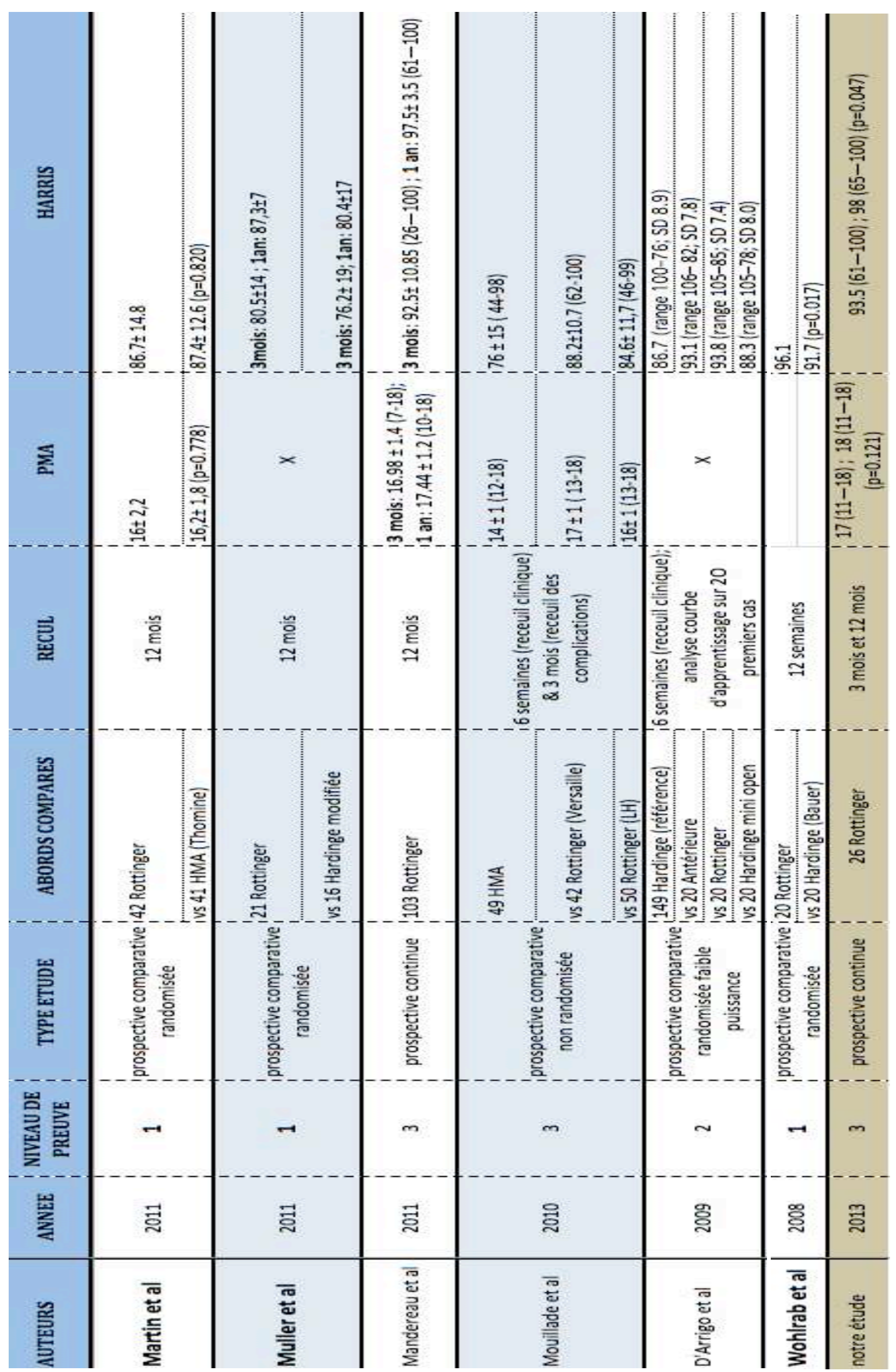

Figure 25: Comparaison des scores cliniques avec la littérature récente $(6,8,56,77,118,119)$ 


\subsubsection{Appui monopodal, boiterie de Trendelenburg et Force musculaire d'abduction.}

Le respect des structures stabilisatrices dynamiques du pelvis, lors de cette chirurgie, semble être confirmé par le faible taux de patients présentant une boiterie de Trendelenburg ainsi qu'un appui monopadal instable et / ou asymétrique (4\%). L'analyse plus fine de ces mauvais résultats fonctionnels, montre qu'il s'agit d'un seul et unique patient qui présentait un appui monopodal instable associé à une boiterie de Trendelenburg à l'examen du $3^{\text {ème }}$ et $12^{\text {ème }}$ mois . A la relecture du dossier médical, il est noté pour ce patient l'existence d'une pathologie douloureuse rachidienne et d'une gonarthrose homolatérale à la hanche opérée. L'existence et l'influence de telles pathologies n'ont cependant pas été étudiées pour l'ensemble de ce travail.

Dans notre étude, les résultats montrent que l'analyse clinique statique (AMP) et dynamique (boiterie de Trendelenburg à la marche) des muscles stabilisateurs du pelvis ne semble pas influencée par l'intervention chirurgicale alors qu'il persiste au $3^{\text {ème }}$ mois une différence significative de la force d'abduction en faveur de la hanche non opérée (Tableaux 3 et 4). L'impact de la voie d'abord sur la mesure de la force d'abduction active de la hanche, en décubitus latéral, semble donc être insuffisante pour avoir une traduction clinique, sur la stabilisation musculaire active du pelvis, en position physiologique d'orthostatisme.

Le bénéfice, en terme de récupération fonctionnelle précoce ( $3^{\text {ème }}$ mois), de cet abord mini invasif, doit être nuancé aux vues des résultats à 12 mois, des deux études comparatives randomisées de 2011:

- Sur le plan statique : Müller et al(77) ont comparé les résultats d'une voie de Röttinger avec ceux d'une voie de Hardinge (121) modifiée « mini open » (incision cutanée < 10 $\mathrm{cm})$. La force d'abduction active était testée en position debout, avec un dynamomètre manuel, fixé à la jambe d'appui au niveau de la cheville. Avec cette méthode, les auteurs (77) n'ont pas retrouvé de différence de force mesurée entre les deux abords comparés, à 3 et 12 mois post opératoire. Néanmoins à 12 mois, ils observaient cliniquement, significativement plus de mauvais résultats pour les caractéristiques de l'AMP («signe de Trendelenburg » (24) ) après réalisation d'un abord transglutéal classique. 
- Sur le plan dynamique: Martin et al (56) ont analysé, les paramètres objectifs caractérisant la marche. Avec un recul de 12 mois, les auteurs n'ont retrouvé aucune différence significative (longueur du pas, phase d'appui, pression du pied, vitesse de marche, bascule frontale du pelvis) entre la voie de Röttinger et l'abord transglutéal classique utilisé ( Hardinge (121) modifiée type Thomine et al(50)).

\subsubsection{Aspect cicatriciel}

Le succès d'une arthroplastie totale de hanche ne dépend pas que de la réussite des temps successifs de l'intervention chirurgicale. Certains paramètres subjectifs et psychologiques doivent également être pris en compte. A partir de questionnaires de satisfaction réalisés chez 165 patients opérés d'une PTH et suivis pendant un an post opératoire, Dorr et al (122) (niveau de preuve 2) ont mis en évidence l'influence psychologique de la taille de l'incision cutanée sur le ressenti des patients. Cette étude pré et post opératoire (6 semaines, 6 mois, 1 an) montrait que les résultats post opératoires attendus (récupération algo -fonctionnelle) après la chirurgie étaient améliorés par l'obtention d'une cicatrice de taille plus petite. Cette constatation était alors en partie expliquée chez $40 \%$ des patients par l'importance de la récupération d'une intégrité de leur schéma corporel.

Ni la taille de la cicatrice, ni la valeur esthétique subjective de celle-ci, n'ont été étudiées dans cette étude. Seul le constat de l'existence d'une «lipodystrophie» ou «lipoatrophie» cicatricielle, sur le trajet de la voie d'abord chirurgicale (Figure 19), a pu être mis en évidence. Cette anomalie, cicatricielle dans notre cas, présente dans la littérature de nombreuses étiologies. Un des traitements possibles semble être la greffe d'adipocytes, comme le transfert de tissus adipeux purifiés atraumatiques (123-125), selon la méthode de Lipostructure ${ }^{\circledR}$ (126-129) de Coleman décrite en 1994, et utilisée par les chirurgiens plasticiens. 


\subsubsection{Modification à l'IRM des muscles stabilisateurs du pelvis.}

Peu d'études d'imagerie évaluant l'aspect qualitatif des muscles fessiers existent dans la littérature $(20,76,103,104)$. L'analyse de la trophicité musculaire et de la dégénérescence graisseuse du muscle petit fessier n'a pas été réalisée dans ce travail. Bien qu'il appartienne à l'appareil abducteur de la hanche et qu'il soit situé à proximité de la voie d'abord chirurgicale. Nous justifions ce choix, par les résultats des travaux des équipes ci-dessous :

- Springer et al (103) ont montré que l'analyse du petit fessier homolatéral à la prothèse reste difficile du fait de la présence d'artefacts métalliques. Les auteurs utilisaient une méthode de contourage manuel des muscles abducteurs et rotateurs externes de hanche après une chirurgie de remplacement arthroplastique bipolaire. Leur analyse sur le volume et la surface de section musculaire montrait une bonne reproductibilité inter et intra observateur (intra meilleure que inter) pour le moyen fessier. Cela n'était pas validé pour le petit fessier.

- Pfirrmann et al (104) ont montré qu'une involution graisseuse du muscle petit fessier semble commune et fréquemment observée à l'IRM. Cette constatation était également vérifiée chez une population de patients asymptomatiques après réalisation d'une chirurgie arthroplastique de hanche. Les auteurs (104) notaient, à l'inverse, que l'observation d'une involution graisseuse du moyen fessier n'était retrouvée que chez les patients symptomatiques d'une boiterie de Trendelenburg ou d'un appui monopodal instable.

La répartition des lésions de dégénérescence graisseuse, observées du côté opéré selon la VALMI, est en accord avec les résultats de Müller et al en 2011(77) (niveau de preuve 1). La VALMI entrainerait globalement moins de grade 3 et 4 que de grades 1 et 2, à 3 et 12 mois post opératoire (Tableau 16). 
Tableau 16: Comparaison des taux de répartition (\%) de la DG du 1/3 antérieure du MF

\begin{tabular}{|l|l|l|l|l|l|l|}
\hline $\begin{array}{l}\text { \% Répartition } \\
\text { dégénérescence graisseuse } \\
\text { côté opéré }\end{array}$ & $\begin{array}{c}\text { Grade 0 : } \\
\text { absence de } \\
\text { graisse }\end{array}$ & $\begin{array}{c}\text { Grade 1 : } \\
\text { quelques } \\
\text { travées } \\
\text { graisseuses }\end{array}$ & $\begin{array}{c}\text { Grade 2 : } \\
\text { graisse }< \\
\text { muscle }\end{array}$ & $\begin{array}{c}\text { Grade 3 : } \\
\text { graisse = } \\
\text { muscle }\end{array}$ & $\begin{array}{c}\text { Grade 4 : } \\
\text { graisse > } \\
\text { muscle }\end{array}$ \\
\hline 3 mois & Müller et al(77) & 38.1 & 23.8 & 28.6 & 9.5 & 0 \\
\cline { 2 - 7 } & Notre étude & 35 & 42 & 19 & 0 & 4 \\
\hline 12 mois & Müller et al(77) & 38.1 & 33.3 & 19 & 4.8 & 4.8 \\
\cline { 2 - 7 } & Notre étude & 58 & 31 & 8 & 4 & 0 \\
\hline
\end{tabular}

Nos résultats montrent que les lésions induites par la voie d'abord sont minimes. Il n'était pas retrouvé d'hypotrophie musculaire significative, secondaire à la chirurgie ; la dégénérescence graisseuse musculaire était globalement peu importante (peu ou pas de grade [3 et 4]).

- Or, la relation entre l'aspect d'un muscle et sa fonction est depuis longtemps étudiée : L'existence d'un principe de corrélation très importante, entre le volume et la force musculaire, fut d'abord démontrée en TDM avec les travaux de Maughan et al en 1983 et $1984(97,98)$, puis confirmée en IRM avec ceux de Akagi et al en 2009 $(130,131)$.

- De plus, Kagan et Abbott (132), ainsi que Lequesne et al.(133), ont montré l'existence d'une relation entre la douleur et la présence de lésions musculo-tendineuses des muscles petit et moyen fessier.

Nous pensons donc que la mise en évidence d'un faible impact lésionnel sur le capital musculaire loco régional du moyen fessier et du TFL du côté opéré par rapport au côté non opéré, nous apporte des éléments de preuve objectifs, quant à la justification de la bonne et rapide récupération algo fonctionnelle après ce type de chirurgie. 


\subsection{Notions générales de capacité régénérative et de compensation musculaire.}

Müller et al (20) ont comparé en 2010, les lésions visibles en IRM, après réalisation d'un abord de Röttinger et d'un abord type Hardinge (121) «modifié mini open ». Dans le groupe des abords transglutéaux classiques, ils ont montré l'existence d'une variation significative de la trophicité musculaire du TFL, au $12^{\text {ème }}$ mois comparativement à l'état préopératoire du muscle. De plus, cette hypertrophie musculaire du TFL était significativement corrélée à l'apparition des lésions de dégénérescence graisseuse du 1/3 antérieur du MF. Les auteurs (20) expliquent cette constatation par la notion d'hypertrophie compensatrice du muscle TFL face à l'agression tissulaire importante, prédominante sur le 1/3 antérieur du MF, lors de la voie d'abord transglutéale type Hardinge.

A l'instar de nos résultats, Müller et al (20) n'ont retrouvé aucune influence de l'abord mini invasif de Röttinger sur la trophicité musculaire du MF et du TFL. Nous confirmons cependant l'existence de lésions infra clinique limitée au 1/3 antérieur du moyen fessier. Cette répartition moins importante de grade 0 («absence de travée graisseuse au sein du muscle ») de la DG, persistant au $12^{\text {ème }}$ mois, peut être expliquée par une «probabilité traumatique instrumentale » plus importante, dans son $1 / 3$ antérieur, au cours des différents temps chirurgicaux (cf Chapitre 2.3 et 3.6.1.3).

Nos résultats montrent également l'existence de lésions plus importantes, prédominant sur le TFL à 3 mois, du côté opéré par rapport au côté non opéré. A 12 mois, l'influence de l'intervention chirurgicale sur le taux de DG n'intervenait significativement, que pour le grade 1 de la DG. Il semble que l'augmentation du taux de grade $2(30 \%$ au lieu de $15 \%$ à 3 mois) du côté non opéré, ait été suffisante pour rendre l'involution graisseuse comparable des deux côtés. Nous pensons que cela met en avant, non pas une notion de cicatrisation musculaire des tissus lésés par la chirurgie, mais plutôt un vieillissement musculaire bilatéral, plus général.

L'influence de l'âge et du sexe de nos patients n'a pas été étudiée dans ce travail. Cependant, deux auteurs ont pu montrer l'influence de ces deux facteurs sur le résultat clinique et IRM de cette chirurgie :

- Dans un autre article, Müller et al (105) en 2011 ont comparé l'influence de la voie d'abord (antéro latérale type Thomine (50) vs VALMI) avec l'âge des patients. Chez 
les patients âgés de plus de 70 ans, les auteurs ont mis en évidence des différences statistiquement significatives pour 3 paramètres étudiés : après 70 ans, les patients opérés par un abord antéro latéral classique présentaient des scores cliniques (Harris et PMA) plus faibles, une augmentation du nombre de signes de Trendelenburg (appui monopodal instable) et un taux de dégénérescence graisseuse plus important, à 3 et 12 mois post op. Aucune différence significative n'a pu être mise en évidence pour ces trois paramètres chez les patients plus jeunes. Les auteurs concluent à l'importance de l'utilisation d'un abord mini invasif chez les patients âgés du fait de leur plus grande fragilité (capacités fonctionnelles post opératoires plus faibles et dégénérescence graisseuse musculaire plus importante) comparativement aux patients jeunes. La corrélation de l'âge et son impact clinique sur le résultat fonctionnel après une chirurgie arthroplastique de hanche avait déjà été montrée dans la littérature (134136). Muller et al (105) expliquent ces constations fonctionnelles cliniques par la mise en évidence d'une corrélation de l'âge avec un taux de dégénérescence musculaire plus important, d'une plus grande vulnérabilité et d'une capacité musculaire régénérative plus faible chez les patient âgés de plus de 70 ans.

- Preininger et al (101), en 2012, ont mis en évidence l'existence d'une relation significativement plus importante pour le sexe masculin entre le volume musculaire et la masse corporelle, par rapport aux femmes. Les auteurs suggèrent le fait que les hommes présenteraient une meilleure capacité d'adaptation de leur masse corporelle. Ils exposent ainsi deux types de conclusions : le volume musculaire des hommes étant mieux corrélé à la masse corporelle, ils possèderaient donc une réserve musculaire plus importante autour de la hanche pour stabiliser le bassin durant la phase d'appui monopodal post opératoire ; Pour les femmes, les auteurs expriment alors l'importance de l'utilisation d'une voie d'abord chirurgicale mini invasive de hanche dans le but d'épargner le capital musculaire des principaux stabilisateurs actifs du pelvis.

Ces différents auteurs, exposent des notions de compensation musculaire et d'adaptation mises en jeu lors de la période post opératoire. Le «vieillissement» musculaire, la « fragilité » globale du patient, son âge et son sexe seraient des facteurs importants et pouvant modifier les capacités de récupération algo-fonctionnelles post opératoire. 


\subsection{Considérations éthiques et information délivrée aux patients}

Les informations disponibles au public sur internet doivent par ailleurs nous laisser critiques. En 2005, Labovitch et Bozic (137) ont analysé les informations disponibles sur internet concernant les voies d'abord mini invasives de hanche. Sur les 150 sites web étudiés, les auteurs montrent que $45 \%$ étaient issus de centres hospitaliers et/ou universitaires, $26 \%$ d'articles peu scientifiques, $25 \%$ de groupes médicaux privés et $6 \%$ de fabricants et industriels orthopédiques. Outre les avantages exprimés et promouvant ces abords mini invasifs, seul $15 \%$ des sites informaient les lecteurs des différentes indications opératoires et des critères d'éligibilité pour ces techniques. L'information des risques et inconvénients n'était expliquée que pour $13 \%$ des sites web. Par ailleurs, $45 \%$ des sites concernés permettaient d'obtenir un rendez vous en ligne. Les auteurs concluaient et insistaient sur l'importance d'une utilisation appropriée des nouveaux moyens de communication, de l'importance morale et éthique de délivrer une information claire et objective quant aux avantages, risques et inconvénients des techniques chirurgicales utilisées. Cette information doit être transmise à la lumière d'études scientifiques bien menées et de niveau de preuve suffisant. En 2008, Wall et al (138) réalisaient une revue de la littérature anglo-saxonne disponible sur PubMed entre 1998 et 2008, à partir de plusieurs mots clefs («minimally invasive hip replacement, minimally invasive hip arthroplasty, mini hip, small incision hip, and small incision hip arthroplasty »). Sur les 172 publications étudiées, seuls neuf essais comparatifs randomisés étaient alors retrouvés. Ces publications réalisaient une comparaison directe entre un abord mini invasif et son abord standard équivalent (postérieur ou latéral). Aucunes d'entre elles ne comparait les abords antérieur et antéro-latéral. La seule différence significative mise en évidence était la diminution du saignement per opératoire $(52 \mathrm{~mL}$ en moyenne) sans qu'il soit prouvé que cela ait un impact clinique. Plus récemment, Hansen et al en 2011 (62) ont réalisé une revue de la littérature centrée sur la voie d'abord antéro latérale de Röttinger. Ils ne citent que 4 études $(56,77,118,120)$ prospectives comparatives randomisées de niveau 1, publiées ou présentées entre 2008 et 2011. Ainsi, outre l'aspect psychologique et subjectif des résultats obtenus après une chirurgie dite mini invasive de hanche, l'apport d'éléments de preuves objectives (cliniques et para cliniques) constitue un véritable enjeu pour notre profession (cf. annexes : Figures 26 et 27 ). 


\section{CONCLUSION}

Cette étude met en évidence de manière objective (clinique et IRM) la notion d'épargne du capital musculaire loco régional avec l'utilisation de cette voie d'abord antéro latérale mini invasive. Elle confirme la faible agression tissulaire de cette voie d'abord de hanche avec une trophicité musculaire conservée et une involution graisseuse peu importante. Les informations objectives apportées par cette analyse descriptive d'une cohorte de patients opérés, pourraient être complétées par un autre travail. L'amélioration de notre méthodologie (cf. Chapitre 5.1.2.5) et la poursuite de la coopération de nos différents services de chirurgie et de radiologie pourraient amener à la réalisation d'une analyse comparative d'autre voies d'abords de hanche (antérieurs pures, et antéro latérale type HMA selon Thomine et al (45)) avec l'association d'une analyse clinique, IRM et sur plateau de marche par exemple. 


\section{BIBLIOGRAPHIE}

1. Woolson ST, Mow CS, Syquia JF, Lannin JV, Schurman DJ. Comparison of primary total hip replacements performed with a standard incision or a mini-incision (English). $\mathbf{J}$ Bone Jt Surg Am Vol. cover date 2004;86A(7):1353-1358.

2. Mardones R, Pagnano MW, Nemanich JP, Trousdale RT. THE FRANK STINCHFIELD AWARD: Muscle Damage after Total Hip Arthroplasty Done with the Two-incision and Mini-posterior Techniques: Clin Orthop. déc 2005;441(\&NA;):63-67.

3. Ogonda L, Wilson R, Archbold P, Lawlor M, Humphreys P, O'brien S, et al. A minimalincision technique in total HIP arthroplasty does not improve early postoperative outcomes : A prospective, randomized, controlled trial. J Bone Jt Surg Am Vol. cover date $2005 ; 87 \mathrm{~A}(4): 701-710$.

4. Meneghini RM, Pagnano MW, Trousdale RT, Hozack WJ. Muscle damage during MIS total hip arthroplasty: Smith-Petersen versus posterior approach. Clin Orthop. 2006;453:293-298.

5. Parratte S, Pagnano MW. Muscle damage during minimally invasive total hip arthroplasty: cadaver-based evidence that it is significant. Instr Course Lect. 2008;57:231-234.

6. Mouilhade F, Matsoukis J, Oger P, Mandereau C, Brzakala V, Dujardin F. Orientation des pièces d'une prothèse totale de hanche de première intention: étude prospective comparative de deux voies d'abord antéro-externes, soit mini-invasive, soit par hémimyotomie du moyen fessier. Compon Position Prim Total Hip Replace Prospect Comp Study Two Antero-Lateral Approaches Minim Invasive Gluteus Medius Hemimyotomy. cover date 2011;97(1):15-24.

7. Levine BR, Klein GR, Di Cesare PE. Surgical approaches in total hip arthroplasty: a review of the mini-incision and MIS literature. Bull NYU Hosp Jt Dis. 2007;65(1):5-18.

8. Mandereau C, Brzakala V, Matsoukis J. Récupération fonctionnelle, complications et mesures scanner de positionnement de prothèses totales de hanche posées par miniabord antérolatéral de Röttinger : à propos d'une série continue de 103 cas. Rev Chir Orthopédique Traumatol. févr 2012;98(1):8-16.

9. Duparc F, Duparc J. Voies d'abord de la hanche pour arthroplastie prothétique de première intention. Open Approach Prim Hip Replace Surg. cover date 2004;85:19-47.

10. Lustig S, Selmi TAS, Michel M, Jacquot L. Chirurgie prothétique de la hanche par voie mini-invasive. EMC - Tech Chir - Orthopédie - Traumatol. janv 2008;3(2):1-10.

11. Duncan C, Toms A, Masri B. Minimally invasive or limited incision hip replacement: clarification and classification. Instr Course Lect. déc 2005;55:195-197.

12. Siguier T, Siguier M, Brumpt B. Mini-incision anterior approach does not increase dislocation rate: a study of 1037 total hip replacements. Clin Orthop. 2004;(426):164-173. 
13. DeWal H, Su E, DiCesare PE. Instability following total hip arthroplasty. Am J Orthop Belle Mead NJ. août 2003;32(8):377-382.

14. Suh KT, Park BG, Choi YJ. A posterior approach to primary total hip arthroplasty with soft tissue repair. Clin Orthop. 2004;(418):162-167.

15. Bertin KC, Röttinger H. Anterolateral mini-incision hip replacement surgery: a modified Watson-Jones approach. Clin Orthop. 2004;(429):248-255.

16. Röttinger H. Minimally invasive anterolateral approach for total hip replacement (OCM technique). Oper Orthop Traumatol. 2010;22(4):421-430.

17. Baker AS, Bitounis VC. Abductor function after total hip replacement. An electromyographic and clinical review. J Bone Joint Surg Br. 1989;71(1):47-50.

18. Madsen MS, Ritter MA, Morris HH, Meding JB, Berend ME, Faris PM, et al. The effect of total hip arthroplasty surgical approach on gait. J Orthop Res. janv 2004;22(1):44.

19. Meneghini RM, Smits SA, Swinford RR, Bahamonde RE. A randomized, prospective study of 3 minimally invasive surgical approaches in total hip arthroplasty: comprehensive gait analysis. J Arthroplasty. 2008;23(6 Suppl 1):68-73.

20. Müller M, Tohtz S, Dewey M, Springer I, Perka C. Evidence of Reduced Muscle Trauma Through a Minimally Invasive Anterolateral Approach by Means of MRI : The 2009 Closed Meeting of the International Hip Society (English). Clin Orthop Relat Res. cover date 2010;468(12):3192-3200.

21. Siebenrock KA, Rösler KM, Gonzalez E, Ganz R. Intraoperative electromyography of the superior gluteal nerve during lateral approach to the hip for arthroplasty. J Arthroplasty. oct 2000;15(7):867-870.

22. Robertson WJ, Gardner MJ, Barker JU, Boraiah S, Lorich DG, Kelly BT. Anatomy and Dimensions of the Gluteus Medius Tendon Insertion. Arthrosc J Arthrosc Relat Surg. févr 2008;24(2):130-136.

23. Flack NAMS, Nicholson HD, Woodley SJ. A review of the anatomy of the hip abductor muscles, gluteus medius, gluteus minimus, and tensor fascia lata. Clin Anat New York N. 2012;25(6):697-708.

24. Trendelenburg F. Trendelenburg's test: 1895. Clin Orthop. 1998;(355):3-7.

25. Al-Hayani A. The functional anatomy of hip abductors. Folia Morphol. mai 2009;68(2):98-103.

26. Gottschalk F, Kourosh S, Leveau B. The functional anatomy of tensor fasciae latae and gluteus medius and minimus. J Anat. cover date 1989;166:179-189.

27. Semciw AI, Green RA, Pizzari T, Briggs C. Verification of a standardized method for inserting intramuscular EMG electrodes into uniquely oriented segments of gluteus minimus and gluteus medius. Clin Anat New York N. mars 2013;26(2):244-252.

28. Neumann DA. Kinesiology of the hip: a focus on muscular actions. J Orthop Sports Phys Ther. févr 2010;40(2):82-94.

29. Dostal WF, Soderberg GL, Andrews JG. Actions of hip muscles. Phys Ther. mars 1986;66(3):351-361. 
30. Semciw AI, Pizzari T, Murley GS, Green RA. Gluteus medius: An intramuscular EMG investigation of anterior, middle and posterior segments during gait. J Electromyogr Kinesiol. août 2013;23(4):858-864.

31. Lewis CL, Sahrmann SA, Moran DW. Anterior hip joint force increases with hip extension, decreased gluteal force, or decreased iliopsoas force. $\mathrm{J}$ Biomech. 2007;40(16):3725-3731.

32. Antonio S, Wolfgang G, Robert H, Fullerton B, Carla S. The anatomical and functional relation between gluteus maximus and fascia lata. J Bodyw Mov Ther. 2013, http://dx.doi.org/10.1016/j.jbmt.2013.04.004

33. Fredericson $\mathrm{M}$, Wolf $\mathrm{C}$. Iliotibial band syndrome in runners: innovations in treatment. Sports Med Auckl NZ. 2005;35(5):451-459.

34. Drake RL, Vogl AW, Mitchell AHG, Duparc F, Duparc J, Co JS\&. Gray's Anatomie pour les étudiants. Elsevier Health Sciences France; 2011.

35. Jacobs LG, Buxton RA. The course of the superior gluteal nerve in the lateral approach to the hip. J Bone Joint Surg Am. sept 1989;71(8):1239-1243.

36. Apaydin N, Kendir S, Loukas M, Tubbs RS, Bozkurt M. Surgical anatomy of the superior gluteal nerve and landmarks for its localization during minimally invasive approaches to the hip. Clin Anat New York N. 2013;26(5):614-620.

37. Standring S. Gray's Anatomy: The Anatomical Basis of Clinical Practice. Churchill Livingstone/Elsevier; 2008.

38. HOLLINSHEAD WH. Textbook of anatomy ... Second edition. Harper \& Row; 1967.

39. Nazarian S, Tisserand P, Brunet C, Müller ME. Anatomic basis of the transgluteal approach to the hip. Surg Radiol Anat. mars 1987;9(1):27-35.

40. Akita K, Sakamoto H, Sato T. The cutaneous branches of the superior gluteal nerve with special reference to the nerve to tensor fascia lata. J Anat. févr 1992;180(Pt 1):105-108.

41. Akita K, Sakamoto H, Sato T. Stratificational relationship among the main nerves from the dorsal division of the sacral plexus and the innervation of the piriformis. Anat Rec. 1992;233(4):633-42.

42. Akita K, Sakamoto H, Sato T. Innervation of the anteromedial muscle bundles of the gluteus medius. J Anat. juin 1993;182(Pt 3):433-438.

43. Akita K, Sakamoto H, Sato T. Arrangement and innervation of the glutei medius and minimus and the piriformis: A morphological analysis. Anat Rec. 1994;238(1):125-30.

44. Akita K, Sakamoto H, Sato T. Origin, Course and Distribution of the Superior Gluteal Nerve. Cells Tissues Organs. 1994;149(3):225-230.

45. Duparc F, Thomine JM, Dujardin F, Durand C, Lukaziewicz M, Muller JM, et al. Anatomic basis of the transgluteal approach to the hip-joint by anterior hemimyotomy of the gluteus medius. Surg Radiol Anat SRA. 1997;19(2):61-67.

46. Moore KL, Dalley AF, Agur AMR. Clinically Oriented Anatomy. Lippincott Williams \& Wilkins; 2013. 
47. Weber M, Ganz R. The Anterior Approach to Hip and Pelvis. Orthop Traumatol. 1 déc 2002;10(4):245-257.

48. Basarir K, Ozsoy MH, Erdemli B, Bayramoglu A, Tuccar E, Dincel VE. The safe distance for the superior gluteal nerve in direct lateral approach to the hip and its relation with the femoral length: a cadaver study. Arch Orthop Trauma Surg. 9 oct 2007;128(7):645-650.

49. Ince A, Kemper M, Waschke J, Hendrich C. Minimally invasive anterolateral approach to the hip: Risk to the superior gluteal nerve. Acta Orthop. févr 2007;78(1):86-89.

50. Thomine JM, Duparc F, Dujardin F, Biga N. Transgluteal approach to the hip by anterior hemimyotomy of the gluteus medius. Rev Chir Orthopédique Réparatrice Appar Mot. sept 1999;85(5):520-525.

51. Lavigne P, Loriot de Rouvray TH. [The superior gluteal nerve. Anatomical study of its extrapelvic portion and surgical resolution by trans-gluteal approach]. Rev Chir Orthopédique Réparatrice Appar Mot. 1994;80(3):188-195.

52. Noble PC, Johnston JD, Alexander JA, Thompson MT, Usrey MM, Heinrich EM, et al. Making minimally invasive THR safe: conclusions from biomechanical simulation and analysis. Int Orthop. 31 juill 2007;31(S1):25-28.

53. Van Oldenrijk J, Hoogland PV, Tuijthof GJ, Corveleijn R, Noordenbos TW, Schafroth MU. Soft tissue damage after minimally invasive THA: A comparison of 5 approaches. Acta Orthop. déc 2010;81(6):696-702.

54. Netter F. Atlas d'anatomie humaine. 5é édition. Masson; 2011.

55. J. Puget; B. Chaminade. Reprise des prothèses de hanche. Elsevier. 2010.

56. Martin R, Clayson PE, Troussel S, Fraser BP, Docquier P-L. Anterolateral minimally invasive total hip arthroplasty: a prospective randomized controlled study with a followup of 1 year. J Arthroplasty. 2011;26(8):1362-1372.

57. Zhang X-L, Shen H, Qin X-L, Wang Q. Anterolateral muscle sparing approach total hip arthroplasty: an anatomic and clinical study. Chin Med J (Engl). 5 août 2008;121(15):1358-1363.

58. Kamath S, Venkatanarasimha N, Walsh MA, Hughes PM. MRI appearance of muscle denervation. Skeletal Radiol. 16 nov 2007;37(5):397-404.

59. Crowe JF, Mani VJ, Ranawat CS. Total hip replacement in congenital dislocation and dysplasia of the hip. J Bone Joint Surg Am. 1979;61(1):15-23.

60. Matsoukis J. Points techniques dans l'arthroplastie de hanche par voie antéro-latérale mini-invasive selon Rottinger. Maitrise Orthopédique N²19. déc 2012;1,14-18.

61. Noble PC, Pflüger G, Junk-Jantsch S, Thompson MT, Alexander JW, Mathis KB. The Optimal Skin Incision for Minimally Invasive Total Hip Arthroplasty Performed via the Anterolateral Approach. J Arthroplasty. juin 2012;27(6):901-908.

62. Hansen BJ, Hallows RK, Kelley SS. The Rottinger approach for total hip arthroplasty: technique and review of the literature. Curr Rev Musculoskelet Med. 2011;4(3):132-138. 
63. Merle d'Aubigné R. Cotation chiffrée de la fonction de la hanche. Rev Chir Orthop. 1970;(56):481-486.

64. Merle d'Aubigne R. Cotation chiffrée de la fonction de la hanche. Rev Chir Orthop Répar Appar Mot. cover date 1990;76(6):371-374.

65. Harris WH. Traumatic arthritis of the hip after dislocation and acetabular fractures: treatment by mold arthroplasty. An end-result study using a new method of result evaluation. J Bone Joint Surg Am. 1969;51(4):737-755.

66. Kijowski R, Blankenbaker DG, Woods MA, Shinki K, Smet AAD, Reeder SB. 3.0-T Evaluation of Knee Cartilage by Using Three-Dimensional IDEAL GRASS Imaging: Comparison with Fast Spin-Echo Imaging1. Radiology. 4 janv 2010;255(1):117-127.

67. Chen CA, Lu W, John CT, Hargreaves BA, Reeder SB, Delp SL, et al. Multiecho IDEAL Gradient-Echo Water-Fat Separation for Rapid Assessment of Cartilage Volume at 1.5 T: Initial Experience1. Radiology. 8 janv 2009;252(2):561-567.

68. Gold GE, Reeder SB, Yu H, Kornaat P, Shimakawa AS, Johnson JW, et al. Articular Cartilage of the Knee: Rapid Three-dimensional MR Imaging at 3.0 T with IDEAL Balanced Steady-State Free Precession-Initial Experience1. Radiology. 8 janv 2006;240(2):546-551.

69. Costa DN, Pedrosa I, Mckenzie C, Reeder SB, Rofsky NM. Body MRI Using IDEAL. Am J Roentgenol 1976. cover date 2008;190(4):1076-1084.

70. Cha JG, Jin W, Lee MH, Kim DH, Park JS, Shin WH, et al. Reducing Metallic Artifacts in Postoperative Spinal Imaging: Usefulness of IDEAL Contrast-enhanced T1- and T2weighted MR Imaging-Phantom and Clinical Studies. Radiology. 6 janv 2011;259(3):885-893.

71. Shapiro L, Harish M, Hargreaves B, Staroswiecki E, Gold G. Advances in musculoskeletal MRI: Technical considerations. J Magn Reson Imaging. 2012;36(4):775-87.

72. Wessig C, Koltzenburg M, Reiners K, Solymosi L, Bendszus M. Muscle magnetic resonance imaging of denervation and reinnervation: correlation with electrophysiology and histology. Exp Neurol. févr 2004;185(2):254-261.

73. Yanny S, Toms AP. MR Patterns of Denervation Around the Shoulder. American Journal of Roentgenology. 2010; 195(2):157-163.

74. Goutallier D, Bernageau J, Patte D. L'évaluation par le scanner de la trophicité des muscles des coiffes des rotateurs ayant une rupture tendineuse. Rev Chir Orthop. 1989;75(Suppl 1):126-7.

75. Goutallier D, Postel JM, Bernageau J, Lavau L, Voisin MC. Fatty muscle degeneration in cuff ruptures. Pre- and postoperative evaluation by CT scan. Clin Orthop. 1994;(304):78-83.

76. Engelken F, Wassilew GI, Köhlitz T, Brockhaus S, Hamm B, Perka C, et al. Assessment of Fatty Degeneration of the Gluteal Muscles in Patients With THA Using MRI: Reliability and Accuracy of the Goutallier and Quartile Classification Systems. J Arthroplasty; http://www.sciencedirect.com/science/article/pii/S0883540313003495 
77. Müller M, Tohtz S, Springer I, Dewey M, Perka C. Randomized controlled trial of abductor muscle damage in relation to the surgical approach for primary total hip replacement: minimally invasive anterolateral versus modified direct lateral approach. Arch Orthop Trauma Surg. 2011;131(2):179-189.

78. Callaghan JJ, Dysart SH, Savory CF, Hopkinson WJ. Assessing the results of hip replacement. A comparison of five different rating systems. J Bone Joint Surg Br. 11 janv 1990;72-B(6):1008-1009.

79. Bryant MJ, Kernohan WG, Nixon, Mollan RA. A statistical analysis of hip scores. J Bone Joint Surg Br. 9 janv 1993;75-B(5):705-709.

80. Bach CM, Feizelmeier H, Kaufmann G, Sununu T, G??bel G, Krismer M. Categorization Diminishes the Reliability of Hip Scores: Clin Orthop. juin 2003;411:166-173.

81. J. Puget. Prothèse totale de hanche, les choix. elsevier; 2005.

82. Constant CR, Murley AH. A clinical method of functional assessment of the shoulder. Clin Orthop. janv 1987;(214):160-164.

83. Frese E, Brown M, Norton BJ. Clinical Reliability of Manual Muscle Testing Middle Trapezius and Gluteus Medius Muscles. Phys Ther. 7 janv 1987;67(7):1072-1076.

84. Cahalan TD, Johnson ME, Liu S, Chao EY. Quantitative measurements of hip strength in different age groups. Clin Orthop. sept 1989;(246):136-145.

85. Asayama I, Chamnongkich S, Simpson KJ, Kinsey TL, Mahoney OM. Reconstructed Hip Joint Position and Abductor Muscle Strength After Total Hip Arthroplasty. J Arthroplasty. juin 2005;20(4):414-420.

86. Ireland M, Wilson J, Ballantyne B, Davis I. Hip strength in females with and without patellofemoral pain. J Orthop Sports Phys Ther. nov 2003;33(11):671-676.

87. Fenter PC, Bellew JW, Pitts TA, Kay RE. Reliability of stabilised commercial dynamometers for measuring hip abduction strength: a pilot study. Br J Sports Med. 8 janv 2003;37(4):331-334.

88. Krause DA, Schlagel SJ, Stember BM, Zoetewey JE, Hollman JH. Influence of Lever Arm and Stabilization on Measures of Hip Abduction and Adduction Torque Obtained by Hand-Held Dynamometry. Arch Phys Med Rehabil. janv 2007;88(1):37-42.

89. Hislop HJ, Montgomery J, Connolly BH. Daniels and Worthingham's muscle testing: techniques of manual examination. WB Saunders Philadelphia, PA; 1995

90. Widler KS, Glatthorn JF, Bizzini M, Impellizzeri FM, Munzinger U, Leunig M, et al. Assessment of Hip Abductor Muscle Strength. A Validity and Reliability Study. J Bone Jt Surg. 1 nov 2009;91(11):2666-2672.

91. Haute Autorité de Santé - Mesure de la force, du travail et de la puissance musculaire, par dynamomètre informatisé et motorisé. Disponible sur: http://www.hassante.fr/portail/jcms/r_1498740/fr/mesure-de-la-force-du-travail-et-de-la-puissancemusculaire-par-dynamometre-informatise-et-motorise 
92. Dwyer MK, Stafford K, Mattacola CG, Uhl TL, Giordani M. Comparison of gluteus medius muscle activity during functional tasks in individuals with and without osteoarthritis of the hip joint. Clin Biomech. (2013), http://dx.doi.org/10.1016/j.clinbiomech.2013.07.007

93. Semciw AI, Pizzari T, Green RA. Technical application and the level of discomfort associated with an intramuscular electromyographic investigation into gluteus minimus and gluteus medius. Gait Posture. mai 2013;38(1):157-160.

94. Semciw, Pizzari T, Green R. Intramuscular EMG placement for two segments of gluteus minimus and three segments of gluteus medius with unique orientation and function. $\mathbf{J}$ Sci Med Sport. déc 2011;14, Supplement 1:e92-e93.

95. Kanehisa H, Ito M, Kawakami Y, Fukunaga T, Miyatani M. The accuracy of volume estimates using ultrasound muscle thickness measurements in different muscle groups. Eur J Appl Physiol. 1 mars 2004;91(2-3):264-272.

96. Sipilä S, Suominen H. Muscle ultrasonography and computed tomography in elderly trained and untrained women. Muscle Nerve. 1993;16(3):294-300.

97. Maughan RJ, Watson JS, Weir J. Strength and cross-sectional area of human skeletal muscle. J Physiol. 1983;338:37-49.

98. Maughan RJ, Watson JS, Weir J. The relative proportions of fat, muscle and bone in the normal human forearm as determined by computed tomography. Clin Sci Lond Engl 1979. 1984;66(6):683-689.

99. Håggmark T, Jansson E, Svane B. Cross-sectional area of the thigh muscle in man measured by computed tomography. Scandinavia Journal od Clinical \& Laboratory Investigation. 1978;38(4): 355-360

100. Preininger B, Schmorl K, von Roth P, Winkler T, Matziolis G, Perka C, et al. More muscle mass in men: explanatory model for superior outcome after total hip arthroplasty. Orthop. févr 2013;42(2):107-113.

101. Preininger B, Schmorl K, von Roth P, Winkler T, Matziolis G, Perka C, et al. The sex specificity of hip-joint muscles offers an explanation for better results in men after total hip arthroplasty. Int Orthop. juin 2012;36(6):1143-1148.

102. Unis DB, Hawkins EJ, Alapatt MF, Benitez CL. Postoperative Changes In The Tensor Fascia Lata Muscle After Using The Modified Anterolateral Approach For Total Hip Arthroplasty. J Arthroplasty. avr 2013;28(4):663-665.

103. Springer I, Müller M, Hamm B, Dewey M. Intra- and interobserver variability of magnetic resonance imaging for quantitative assessment of abductor and external rotator muscle changes after total hip arthroplasty. Eur J Radiol. 2012;81(5):928-933.

104. Pfirrmann CWA, Notzli HP, Dora C, Hodler J, Zanetti M. Abductor tendons and muscles assessed at MR imaging after total hip arthroplasty in asymptomatic and symptomatic patients. Radiology. 2005;235(3):969-976.

105. Müller M, Tohtz S, Dewey M, Springer I, Perka C. Age-related appearance of muscle trauma in primary total hip arthroplasty and the benefit of a minimally invasive approach for patients older than 70 years. Int Orthop. 2 déc 2010;35(2):165-171. 
106. Rasch A, Byström AH, Dalen N, Berg HE. Reduced muscle radiological density, crosssectional area, and strength of major hip and knee muscles in 22 patients with hip osteoarthritis. Acta Orthop. janv 2007;78(4):505-510.

107. Mattei JP, Fur YL, Cuge N, Guis S, Cozzone PJ, Bendahan D. Segmentation of fascias, fat and muscle from magnetic resonance images in humans: the DISPIMAG software. Magma New York N. 2006;19(5):275-279.

108. Lamminen AE, Tanttu JI, Sepponen RE, Suramo IJ, Pihko H. Magnetic resonance of diseased skeletal muscle: combined T1 measurement and chemical shift imaging. Br J Radiol. août 1990;63(752):591-596.

109. Huppertz A, Radmer S, Asbach P, Juran R, Schwenke C, Diederichs G, et al. Computed tomography for preoperative planning in minimal-invasive total hip arthroplasty: Radiation exposure and cost analysis. Eur J Radiol. juin 2011;78(3):406-413.

110. Kalteis T, Handel M, Herold T, Perlick L, Paetzel C, Grifka J. Position of the acetabular cup-accuracy of radiographic calculation compared to CT-based measurement. Eur J Radiol. mai 2006;58(2):294-300.

111. Rudisch A, Kremser C, Judmaier W, Zunterer H, DeVries AF. Dynamic contrastenhanced magnetic resonance imaging: a non-invasive method to evaluate significant differences between malignant and normal tissue. Eur $\mathbf{J}$ Radiol. mars 2005;53(3):514-519.

112. Anderson SE, Siebenrock KA, Mamisch TC, Tannast M. Femoroacetabular Impingement Magnetic Resonance Imaging: Top Magn Reson Imaging. juin 2009;20(3):123-128.

113. Kassarjian A, Cerezal L, Llopis E. [MR arthrography of the hip with emphasis on femoroacetabular impingement]. Radiologia. févr 2009;51(1):17-29; quiz 119.

114. Toms AP, Smith-Bateman C, Malcolm PN, Cahir J, Graves M. Optimization of metal artefact reduction (MAR) sequences for MRI of total hip prostheses. Clin Radiol. juin 2010;65(6):447-452.

115. Holmbäck AM, Askaner K, Holtås S, Downham D, Lexell J. Assessment of contractile and noncontractile components in human skeletal muscle by magnetic resonance imaging. Muscle Nerve. 2002;25(2):251-258.

116. Rasband. W. ImageJ [Internet]. editor.ImageJ.Bethesda,MD:U.S.National Institutes of Health; 1997. Disponible sur: http://imagej.nih.gov/ij/

117. Goutallier D, Postel JM, Lavau L, Bernageau J. Influence de la dégénérescence graisseuse des muscles supraépineux et infraépineux sur le pronostic des réparations chirurgicales de la coiffe des rotateurs. Influ Supraspinatus Infraspinatus Muscular Fat Degener Progn Rotator Cuff Surg Repair. cover date 1999;85(7):668-676.

118. Wohlrab D, Droege J-W, Mendel T, Brehme K, Riedl K, Leuchte S, et al. [Minimally invasive vs. transgluteal total hip replacement. A 3-month follow-up of a prospective randomized clinical study]. Orthop. nov 2008;37(11):1121-1126.

119. D'Arrigo C, Speranza A, Monaco E, Carcangiu A, Ferretti A. Learning curve in tissue sparing total hip replacement: comparison between different approaches. J Orthop Traumatol Off J Ital Soc Orthop Traumatol. mars 2009;10(1):47-54. 
120. White R. Early performance of the posterolateral mini approach and the Watson-Jones anterolateral approach- a blinded, randomized clinical trial. Las Vegas, Nevada; 2009.

121. hardinge kevin. the direct lateral approach to the hip. Direct Lateral Approach Hip J Bone Jt Surg Br 6417-19.

122. Dorr LD, Thomas D, Long WT, Polatin PB, Sirianni LE. Psychologic reasons for patients preferring minimally invasive total hip arthroplasty. Clin Orthop. mai 2007;458:94-100.

123. Lewis CM. Correction of deep gluteal depression by autologous fat grafting. Aesthetic Plast Surg. 1992;16(3):247-250.

124. Lewis CM. Comparison of the syringe and pump aspiration methods of lipoplasty. Aesthetic Plast Surg. déc 1991;15(1):203-208.

125. Mojallal A, Breton P, Delay E, Foyatier J-L. Greffe d'adipocytes : applications en chirurgie plastique et esthétique. EMC - Chir. avr 2005;2(2):175-203.

126. Coleman SR. Structural fat grafts: the ideal filler? Clin Plast Surg. janv 2001;28(1):111-119.

127. Coleman SR. Hand rejuvenation with structural fat grafting. Plast Reconstr Surg. déc 2002;110(7):1731-1744; discussion 1745-1747.

128. Coleman SR. Long-term survival of fat transplants: controlled demonstrations. Aesthetic Plast Surg. oct 1995;19(5):421-425.

129. Coleman SR. Facial recontouring with lipostructure. Clin Plast Surg. avr 1997;24(2):347-367.

130. Akagi R, Takai Y, Ohta M, Kanehisa H, Kawakami Y, Fukunaga T. Muscle volume compared to cross-sectional area is more appropriate for evaluating muscle strength in young and elderly individuals. Age Ageing. 2009;38(5):564-569.

131. Akagi R, Takai Y, Kato E, Fukuda M, Wakahara T, Ohta M, et al. Relationships Between Muscle Strength and Indices of Muscle Cross-Sectional Area Determined During Maximal Voluntary Contraction in Middle-Aged and Elderly Individuals: J Strength Cond Res. juill 2009;23(4):1258-1262.

132. Kagan, abbott. Rotator Cuff Tears of the Hip: Clinical Orthopaedics and Related Research. nov 1999;368.

133. Lequesne M, Djian P, Mathieu P, Vuillemin-Bodaghi V. Étude prospective des tendinobursites trochantériennes rebelles. Images IRM des ruptures tendineuses glutéales opérées. Résultats cliniques et IRM des sutures. Rev Rhum. juill 2008;75(7):640-646.

134. Röder C, Parvizi J, Eggli S, Berry DJ, Müller ME, Busato A. Demographic factors affecting long-term outcome of total hip arthroplasty. Clin Orthop. 2003;(417):62-73.

135. Johnsson R, Thorngren KG. Function after total hip replacement for primary osteoarthritis. Int Orthop. 1989;13(4):221-225.

136. Pettine KA, Aamlid BC, Cabanela ME. Elective total hip arthroplasty in patients older than 80 years of age. Clin Orthop Relat Res. cover date 1991;(266):127-132. 
137. Labovitch RS, Bozic KJ, Hansen E. An Evaluation of Information Available on the Internet Regarding Minimally Invasive Hip Arthroplasty. J Arthroplasty. janv 2006;21(1):1-5.

138. Wall SJ, Mears SC. Analysis of published evidence on minimally invasive total hip arthroplasty. J Arthroplasty. oct 2008;23(7 Suppl):55-58. 


\section{ANNEXES}

Tableau 17: évaluation chiffrée PMA (Postel, Merle d'Aubigné)

\begin{tabular}{|c|c|c|c|}
\hline & Douleur & Mobilité amplitude en flexion & Marche / stabilité \\
\hline 6 & Aucune & $\geq 90^{\circ}$ & Normale ou illimitée \\
\hline 5 & Rare et légère & $80-70^{\circ}$ & $\begin{array}{l}\text { Limitée } \\
\text { Légère boiterie si prolongée } \\
\text { Cannes pour longues distances } \\
\text { Pas d'instabilités }\end{array}$ \\
\hline 4 & 30min à $1 \mathrm{~h}$ & $70-50^{\circ}$ & $\begin{array}{l}\text { Canne toujours pour sortir } \\
\text { Boiterie nette } \\
\text { Légère instabilité }\end{array}$ \\
\hline \multicolumn{4}{|c|}{$\begin{array}{l}\text { Apparaissant à la marche au } \\
\text { bout de: }\end{array}$} \\
\hline 3 & $1-20 \mathrm{~min}$ & $50-30^{\circ}$ & $\begin{array}{l}\text { Cannes en permanence } \\
\text { Instabilité majeure }\end{array}$ \\
\hline 2 & Avant 10min & $<30^{\circ}$ & Deux cannes \\
\hline 1 & Immédiatement & & Béquilles \\
\hline $\mathbf{0}$ & $\begin{array}{l}\text { Permanente même en } \\
\text { position assise ou couchée }\end{array}$ & $\begin{array}{l}-1 \text { si attitude vicieuse flexion/rotation } \\
\text { externe } \\
-2 \quad \text { si attitude vicieuse } \\
\text { abduction/adduction/rotation interne }\end{array}$ & Impossible \\
\hline
\end{tabular}


Tableau 18: cotation fonctionnelle de Harris

\begin{tabular}{|c|c|c|}
\hline Douleur & Activités & Mobilité (en degrés) \\
\hline Aucune : 44 & Escaliers & Flexion: \\
\hline Minime : 40 & Normal : 4 & Abduction: \\
\hline Légère : 30 & Avec rampes : 5 & Adduction: \\
\hline Modérée : 20 & Asymétrique : 1 & Rotation interne : \\
\hline Importante : 10 & Impossible : 0 & Rotation externe: \\
\hline Très importante : 0 & Bas-chaussures & Total : \\
\hline Marche & Facilement :4 & Total en degrés : \\
\hline Boiterie & Difficilement : 5 & $210-300^{\circ}: 6$ \\
\hline Aucune : 11 & Impossible : 0 & $160-210^{\circ}: 5$ \\
\hline Légère : 8 & S'asseoir & $100-160^{\circ}: 4$ \\
\hline Modérée : 5 & Toute chaise $1 \mathrm{~h}: 4$ & $60-100^{\circ}: 3$ \\
\hline Sévère : 0 & Chaise haute $\leq 0,5 \mathrm{~h}: 2$ & $30-60^{\circ}: 1$ \\
\hline Aide & Toute chaise $\leq 0,5 \mathrm{~h}: 0$ & $0-30^{\circ}: 0$ \\
\hline Aucune : 11 & Transport en commun & Score total \\
\hline Canne longue marche : 7 & Possible : 1 & Douleur (44) : \\
\hline Canne toujours : 5 & Impossible : 0 & Fonction (33) : \\
\hline Béquille : 4 & Si aucune de ces anomalies : 4 & Activités (13): \\
\hline Deux cannes : 2 & Si ou plus de ces anomalies : 0 & Pas d'anomalie (4): \\
\hline Deux béquilles : 0 & Adduction fixée $\geq 10^{\circ}$ & Mobilité (6) : \\
\hline Périmètre de marche & Flexion fixée $\geq 30^{\circ}$ & Total (100): \\
\hline Illimité : 11 & Rotation interne fixée $\geq 10^{\circ}$ & \\
\hline$<1 \mathrm{~km}: 8$ & Inégalité de longueur $\geq 2-3 \mathrm{~cm}$ & \\
\hline \multicolumn{3}{|l|}{$<500 \mathrm{~m}: 5$} \\
\hline \multicolumn{3}{|l|}{ A l'intérieure : 2} \\
\hline Lit-fauteuil : 0 & & \\
\hline
\end{tabular}




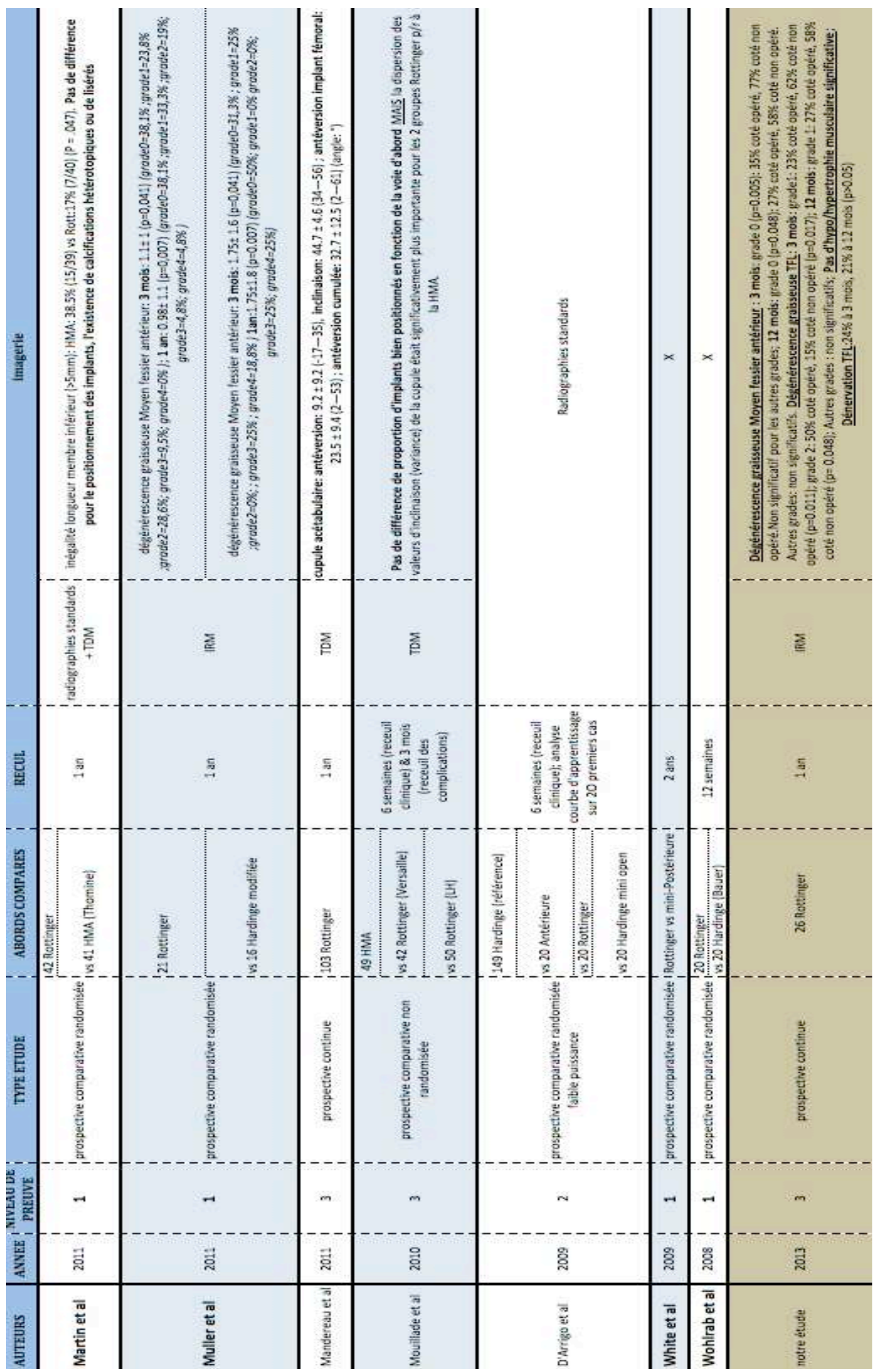

Figure 26: Comparaison des résultats paracliniques avec la littérature récente $(6,8,56,77,118,119)$ 


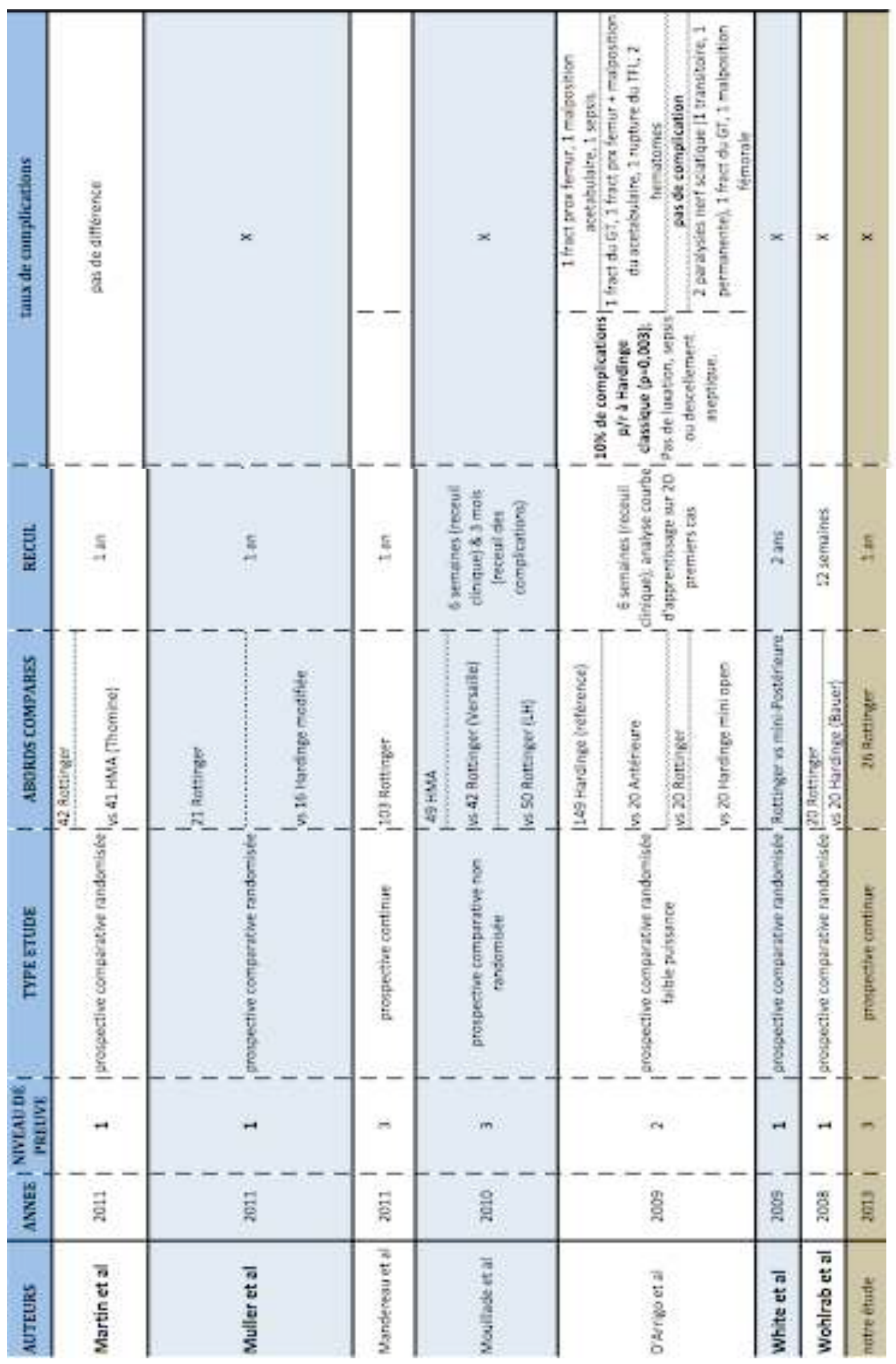

Figure 27:Comparaison des taux de complications dans la littérature récente $(6,8,56,77,118,119)$ 

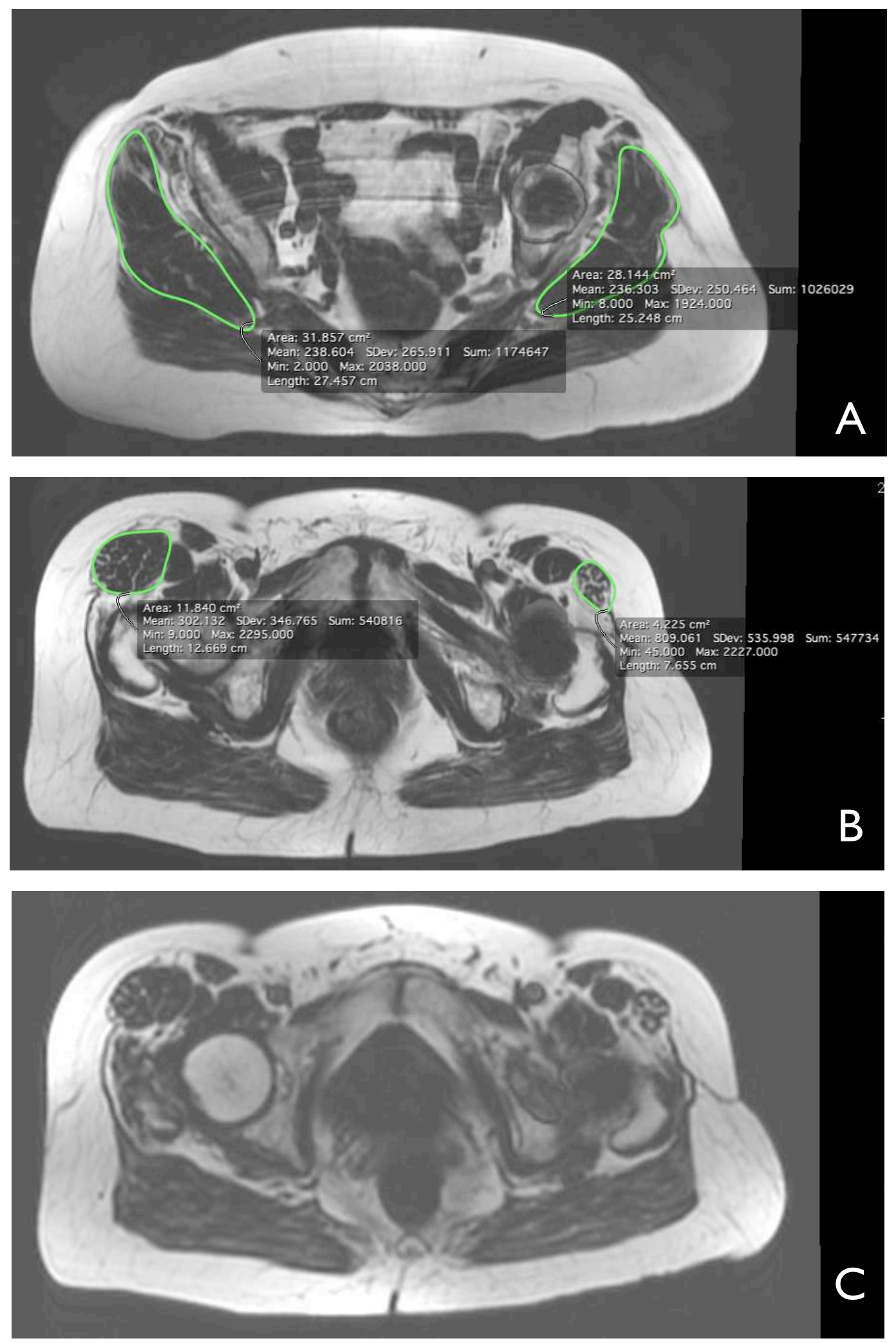

Figure 28: exemple de dénervation du TFL

\section{A : coupe FAT Ax T1 IDEAL FSE, évaluant le MF}

(c. opéré : $28.14 \mathrm{~cm}^{2}$, grades : $1 / 1 / 0$; c. non opéré : $31.86 \mathrm{~cm}^{2}$; grades : 2/1/1)

B : coupe FAT Ax T1 IDEAL FSE, évaluant le TFL

(c. opéré : $4.23 \mathrm{~cm}^{2}$, grades : 3 ; c. non opéré : $11.84 \mathrm{~cm}^{2}$; grades : 1)

\section{C : coupe FAT Ax T2 IDEAL FSE, évaluant le TFL :}

hypersignal $\mathrm{T} 2$ de d'oedème de dénervation 


\section{Résumé}

\section{Introduction}

L'absence de section musculaire lors du remplacement arthroplastique total de hanche par abord antéro latéral mini invasif ne garantit pas l'absence de lésion musculo-nerveuse infraclinique, dépistable à l'IRM. Le but de cette étude était l'évaluation objective du respect du capital musculaire du moyen fessier (MF) et du tenseur du fascia lata (TFL), par une étude clinique et IRM, à 3 et 12 mois post opératoire, comparativement au côté non opéré.

\section{Matériel et méthode}

L'étude prospective, mono opérateur, incluait une série continue de 26 patients opérés entre le $1^{\text {er }}$ avril et le 6 décembre 2011. Le suivi comportait la réalisation d'un bilan clinique (score de Harris et PMA, recherche d'une boiterie de Trendelenburg, caractéristiques de l'appui monopodal, mesure de la force d'abduction active de hanche au dynamomètre) ainsi qu'une IRM de bassin, à 3 et 12 mois post opératoire. Les 2 imageries analysaient la trophicité musculaire, la dégénérescence graisseuse du MF et du TFL et l'existence d'un oedème de dénervation du TFL.

\section{Résultats}

L'analyse des scores cliniques confirmait la récupération fonctionnelle rapide, dès 3 mois: PMA médian $(M 3 / M 12)=17 / 18$ [11-18]. Une amélioration significative du score de Harris était retrouvée entre 3 et 12 mois (Harris médian M3/M12=93.5 [61 - 100]/ 98 [65-100], $\mathrm{p}=0.047)$. La mesure dynamométrique de la force d'abduction des 2 hanches était significativement meilleure du côté non opéré au $3^{\text {me }}$ mois $(\mathrm{p}=0.038)$ sans différence retrouvée entre les 2 hanches à 12 mois. Cette différence de force avait peu de traduction clinique : $92 \%$ des patients présentaient un appui monopodal stable et symétrique et 1 seul patient gardait une boiterie de Trendelenburg à 12 mois. L'influence de la voie d'abord sur l'apparition de la dégénérescence graisseuse du MF, était limitée à son $1 / 3$ antérieur (M3, $\mathrm{p}=0.005 ; \mathrm{M} 12, \mathrm{p}=0.048$ ) et de manière peu importante (côté opéré M3 : grade $0 / 1 / 2 / 3 / 4=$ $35 \% / 42 \% / 19 \% / 0 \% / 4 \%$; côté non opéré M12 : grade 1/2/3/4 = 19\%/4\%/ 0\%/ 0\%, NS). A 3 mois, la dégénérescence graisseuse du TFL présentait significativement moins de grade 1 et plus de grade 2 du côté opéré (grade 1 opéré/non opéré : $23 \% / 62 \%, \mathrm{p}=0.011$; grade 2 opéré/non opéré : $50 \% / 15 \%$, p=0.017). A 12 mois, il persistait moins de grade 1 dans le TFL opéré (grade 1 opéré/non opéré : $27 \% / 58 \%, \mathrm{p}=0.048$ ), aucune influence de la voie d'abord n'était retrouvée pour les autres grades. L'existence de lésions de dénervation était confirmée dans $21 \%$ des cas, à 12 mois. Aucune influence de la voie d'abord sur la trophicité musculaire du MF et du TFL n'était retrouvée à 12 mois.

\section{Conclusion}

Cette étude confirme objectivement la faible agression tissulaire générée par la voie d'abord.

\section{Niveau de preuve 3, Etude prospective continue.}

Mots clefs : Prothèse Totale de Hanche, Imagerie par Résonnance Magnétique, Lésions muscles abducteurs, Abord antéro latéral mini invasif, Muscle moyen fessier, Tenseur du fascia lata. 